1.5.

1
1

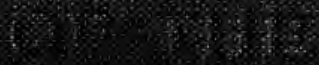

15.2.

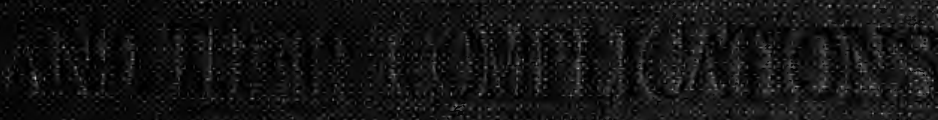

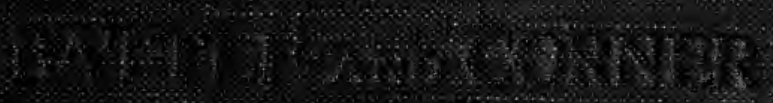

策

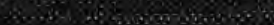

2.8.

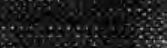




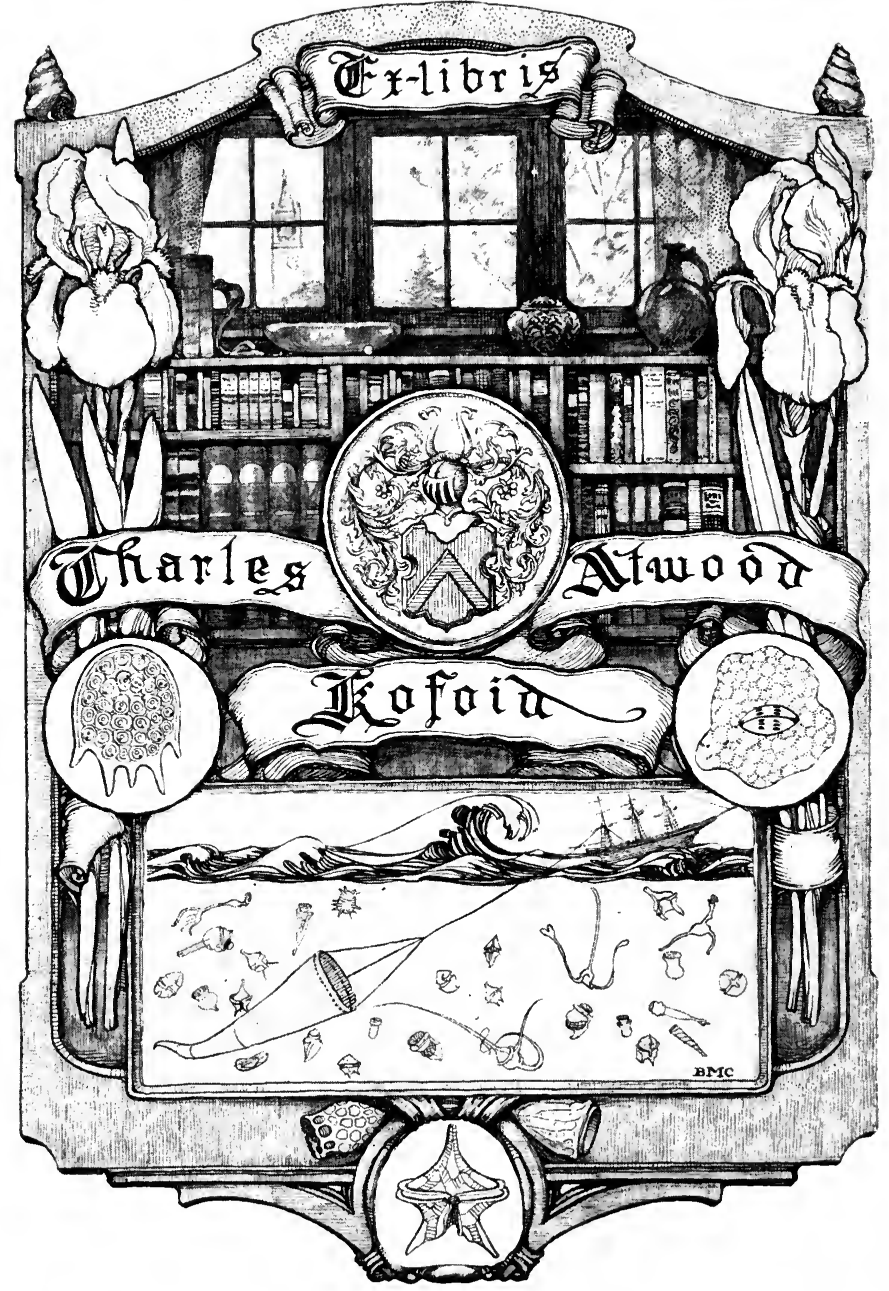




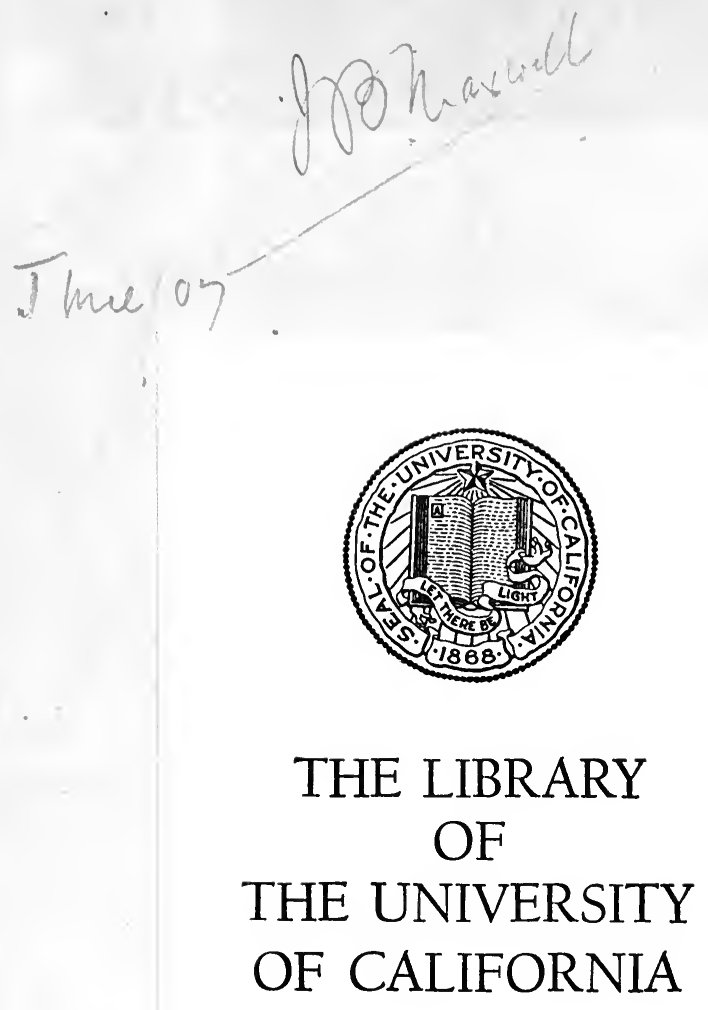

PRESENTED BY

PROF. CHARLES A. KOFOID AND MRS. PRUDENCE W. KOFOID 





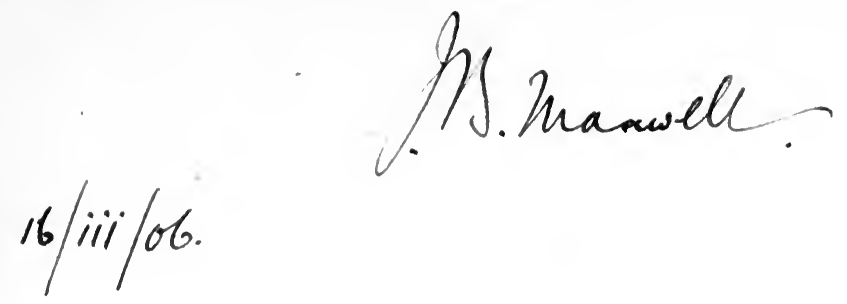

THE SURGERY OF THE DISEASES OF THE APPENDIX VERMIFORMIS AND THEIR COMPLICATIONS 

Digitized by the Internet Archive in 2007 with funding from Microsoft Corporation 


\section{STRANGULATION OF APPENDIX IN A FEMORAL SAC.}

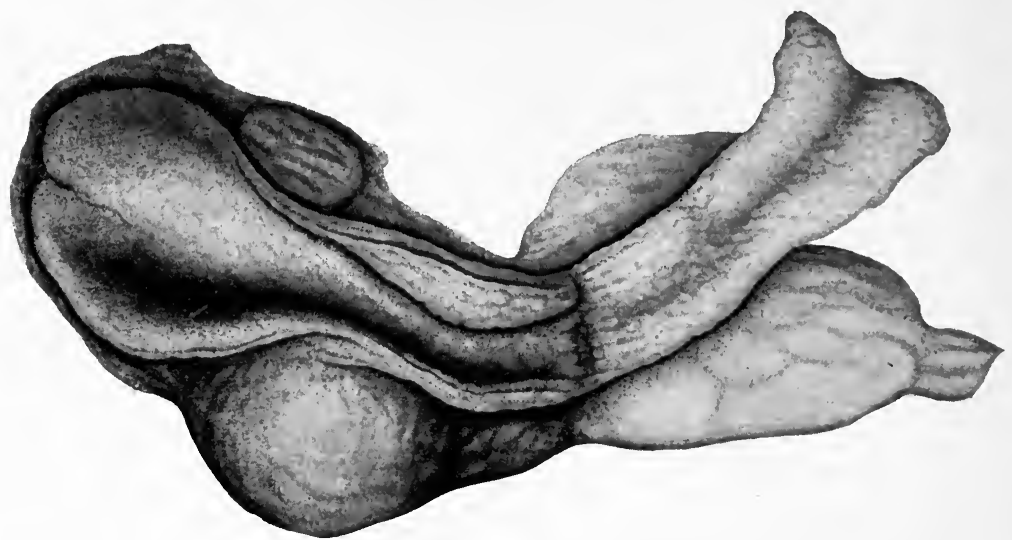

FIG. I.-Shows the swollen inflamed distal encl of the Appendix separated by a sharp line of demarcation from the healthy proximal part. The Mesentery is also shown thickened and inflamed. The Mucous Membrane is also seen to be increased in thickness.

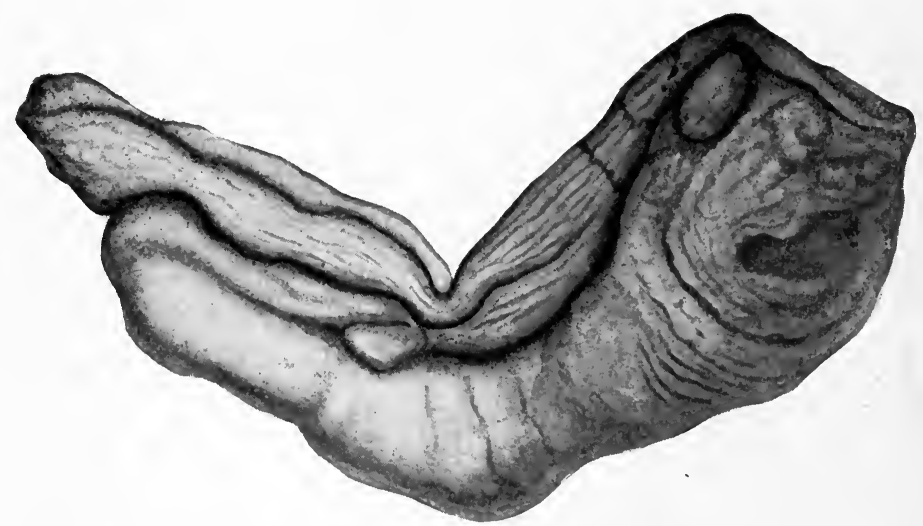

FIG. 2.- Shows the perforation close to the tip of the Appendix where it is most swollen. The line of Constriction of the Mesentery is also well seen.

By permission of the Editors of St. Thomas' Hospital Reports-from the paper by Messrs. Corner \& Spurrier on the Relation of the Appendix and Meckel's diverticulum to Hernia Sacs, 1902. 


\section{The Surgery of the Diseases of the Appendix Vermiformis and their Complications}

$\mathrm{BY}$

WILLIAM HENRY BATTLE, F.R.C.S

Surgeon to St. Thomas' Hospital, formerly Surgeon to the Royal Free Hospital, Hunterian Professor of Surgery at the Royal College of Surgeons of England, etc

AND

EDRED M. CORNER, M.B., B.C., F.R.C.S

Surgeon in charge of Out Patients to St. Thomas' Hospital and Assistant Surgeon to the Great Ormond Street Hospital for Sick Children, Erasmus Wilson Lecturer at the Royal College of Surgeons, etc

LONDON

ARCHIBALD CONSTABLE $\xi$ CO LTD

I 6 JAMES STREET HAYMARKET

1904 
Butler \& Tanner,

The Selwood Printing Works,

Frome, and Lundon. 


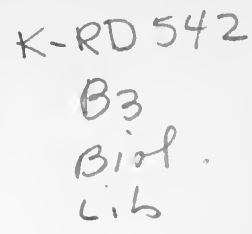

\section{Preface}

The literature on the subject of the Appendix Vermiformis and its diseases has become so extensive that it is now quite impossible for any one without access to a large medical library, and abundance of leisure, to obtain an adequate idea of the subject. During the past few years many hundreds of communications of very varying value in this department of medicine have appeared in the medical press of the world. It has been a task of some magnitude to look through the contributions and consult the more important monographs, and we fear that even now it may appear to some that their work has not received its due recognition at our hands. We have tried to write impartially from the standpoint of the practical Surgeon, before whom a constant stream of illustrative cases is passing. We have therefore summarized, as briefly as the importance of the subject permits, the views held by the Physician and the Pathologist, and tried to place the Surgeon's view before the profession in such a way that it shall be of practical value.

Further, we have included sections on Acute Abdominal Disease, Carcinoma, Tubercle and other diseases of the Appendix, Life Insurance, etc., which have not formed a place in previous publications on the subject.

We beg to thank our colleague, Dr. Hector Mackenzie, for valuable assistance and suggestions, and Mr. G. W. Ord, of Richmond, for some of the illustrations to the text. 



\section{Contents}

OHAP.

PAGE

I History of the Disease and its Surgical Treatment - The Anatomy of the Appendix Vermiformis, Macroscopical, Microscopical; The Peritoneal Fosse; The Abdominal Wali and its RelationShIP to the Appendix-Physiology-DeVelopment

II Atiology : Pathology : Chronic, Acute (Non-Suppurative; with Local Suppuration, with Peritonitis) - The Structure and Formation of Concretions -Leucocytosis-Bacteriology

III Non-Suppurative Appendicitis : Acute, Chronic: Symptoms, Diagnosis, Treatment-Appendicitis From Torsion-ApPendicular Colic . . .

IV The Various Methods of Operation for the Removal of the Appendix Vermiformis in the Quiet Period

V The Complications of Appendicitis-Circumscribed Suppuration: Appendix Abscess, Perityphlitic

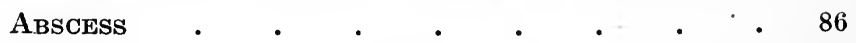

VI Appendicitis with Spreading Peritonitis-Value of INDIVIDUAL SYMPTOMS $\quad . \quad . \quad . \quad . \quad .106$

VII The Treatment of Diffuse Peritonitis . . . 122

ViII The Diagnosis of the Acute Abdominal Conditions which Require Surgical Treatment . . . . 134 
CHAP.

IX Intussusception--Some of the Rarer Diseases of the Appendix-Benign Tumours. SarcomaCarcinoma-Actinomycosis-Tuberculosis . 154

$\mathrm{X}$ Complications of Appendicitis (continued) : Subdiaphragmatic and other Secondary Abscesses -Empyama and other Lung Diseases-Cardiac and Renal Affections - Thrombosis of Veins -Hamorrhages-Chronic Cellulitis of the Right Iliac Fossa-Myositis of the Iliacus and PsoAs . . . . . . .

XI Complications of Appendicitis (continued)-Pelvic Complications : Salpingitis, Pyosalpynx, Pregnancy-Intestinal Obstruction-Fistula (External and Internal)-The Relation of the Appendix to Hernia-Effects of the ToxinesHYPOCHONDRIASIS AND NEURASTHENIA-RHEUMATISM AND Gout-SyphILIS AND GoNorRHGa-APPENDIcitis with Dysenteric, Typhoid and Stercoral

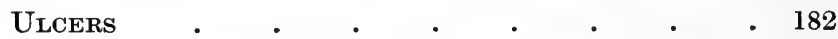

XII Selection for Life Insurance $\quad . \quad \ldots \quad$. $\quad . \quad 199$ 


\section{List of Illustrations and Diagrams}

FIG.

PAGE

Strangulation of Appendix in a Femoral Sac Frontispiece

1 The Peritoneal Fossa about the Appendix . . 20

2 Types of Development of the Cecum . • . . . 28

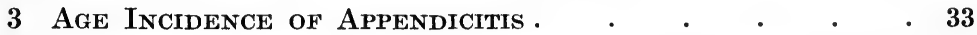

4 Representing the Relationship of the Appendix to the Position of the Ureter and Abdominal Wall • 62

5 To Illustrate the Position of the Superficial Incision with Regard to the Rectus Muscle $\quad . \quad$. $\quad . \quad$. 77

6 Clanp . 6 . 6 .

7 The Extent of the Application of the Clamp on the

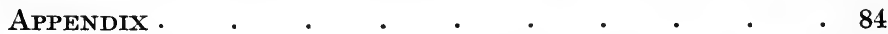

8 Appendix (half-size) showing Localized Gangrene with Perforation Secondary to a Facal Concretion (see p. 109) .

9 Position and Relative Size of Concretion as shown by

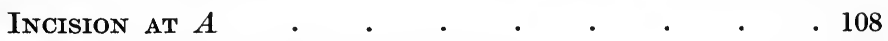

10 Intussusception of the Appendix • • • • • 155 



\section{CHAPTER I}

History of the Disease and of its Surgical Treatment. The Anatomy of the Appendix Vermiformis, Macroscopical, Microscopical. The Peritoneal Fossae; The Abdominal Wall and its Relationship to the Appendix-The Physiology of the Appendix-Its Development.

\section{HISTORY}

THE history of a disease is always of the greatest interest, and the study of it is most wholesome in that it illustrates, often in a striking manner, that more progress is made through unsuccessful than successful cases. With the early tide of success the progress of pathological knowledge, which is the sound basis of treatment, becomes slow in consequence of its growth being stifled by the hue and cry of clinical triumphs. The history of appendicitis has passed, we hope, through these stages, and now we are at the dawn of a further period of scientific work. In consequence, it is no easy matter to write an adequate yet brief account of the history of the disease, and we trust that our shortcomings in this direction will be excused on the ground of difficulty rather than lack of appreciation or slight of the good work of our predecessors.

Deaver, in his text-book, has pointed out that three distinct periods may be recognized. In the first, the most striking lesions, such as perforation and gangrene of the appendix, are reported as examples of mere pathological interest ; in the second, the appendix appears of very secondary importance to the caecum; and in the third, that structure is 


\section{SURGERY OF THE APPENDIX}

regarded as the fons et origo mali of almost every ill in the right iliac fossa. To these we should like to add a fourth stage, which recognizes the paramount importance of the infective organisms which are at work, the great frequency of chronic or subacute appendicitis, and that the involvement of the caecum is almost invariably secondary to that of the appendix.

Saracenus, in 1642, and Mestivier, in 1759, report the earliest recognizable cases of appendicitis with abscess. Whilst it is probable that Heister, in 1711, was the first to accurately observe the relationship between disease of the appendix and diffuse peritonitis. ${ }^{1}$

In the British Isles, Dr. Parkinson, in 1812, described the first case of appendicitis and general peritonitis. Dr. Copland, in 1832, discriminated between inflammation of the caecum, inflammation of the appendix, and inflammation of the pericæcal tissue. In 1836, Dr. Hodgkin recognized that the appendix was responsible for both local abscesses of the right iliac fossa and diffuse peritonitis. Grisolle, in 1839, reported sixty cases in which he believed the appendix was responsible for the inflammation in the right iliac fossa. Rokitansky, in 1855 , was the first to fully consider the subject of catarrhal and chronic appendicitis with their relationship to concretions. The subject was then taken up by the American surgeons, who have done so much to further our knowledge of the disease that it seems almost invidious to select any for special mention. But the work of Fitz, embodied chiefly in two papers, published in 1886 and 1888 respectively, cannot be passed over. Nor should the first reported successful operation on acute appendicitis with spreading peritonitis by Sands be unnoticed. And to McBurney we owe the first advocacy of early operation in acute cases. Besides these names, we may recall those of Parker, Richardson, Murphy, Price, Forster, Morris, and yet 1 Mr. W. Wright, Brit. Med. Journ., Sept. 26, 1903. 
the multitude of excellent workers is too great for each to be individually mentioned. For in the history of this disease, more than in any other, have the American surgeons most clearly left their mark.

In England also there have been and are a multitude of workers, but it may be pointed out that Sir Samuel Wilks, in 1875 , insisted on the great importance of the rôle which the appendix played in the production of local abscesses and diffuse peritonitis. But the subject had never been adequately treated in this country until the publication in 1895 of Dr. Hawkins' classical monograph.

From this date the literature seems more to have been given over to clinical successes rather than to the elucidation of pathological problems. And numerous monographs have sprung up in all countries and languages.

The subject of the history of operations for appendicitis is one of which but a short account need be given. Mr. Hancock, in 1848, reported the first case of the incision of an appendix abscess, before fluctuation was present, in England. It is but little known that to Mr. Charters Symonds, in conjunction with Dr. Mahomed, belongs the credit of planning and successfully carrying out the first operation, during the quiet interval, for the relief of symptoms due to disease of the appendix, which was undertaken in this country. But it has been chiefly owing to the strenuous and excellent work of Sir Frederick Treves that the operation has been demonstrated to be as safe and sure as any within the range of surgical procedures. On the other side of the Atlantic, the surgeons have led in the adoption of almost every surgical measure that has been utilized for appendicitis. And it seems now necessary only to pay this tribute to their work.

The surgical treatment of appendix abscesses was naturally the first to be evolved. Abscesses were epened long before their origin was known. The rational treatment of appen- 
dicitis with peritonitis is quite a modern procedure, and is yet in the flush of that clinical success in which pathological problems are obscured by questions of technique.

\section{ANATOMY}

The gross anatomy of the appendix will be most easily and briefly considered in a series of short sections.

The appendix is, when normal, of a yellowish pink colour, and is quite soft and pliable. In the perfectly healthy organ no contents are to be felt within it, and any deviation from this goes in company with disease of the organ.

The average length is $8-9$ centimetres, or $3 \frac{1}{2}$ inches, but it may be as much as 11 inches long, or as short as half an inch. It has been shown by Ribbert, Berry, and others that the appendix is slightly longer and thicker in the male than in the female.

In diameter it varies, and is on the average $6 \mathrm{~mm}$. thick in the male and $5 \mathrm{~mm}$. in the female.

It has been shown that the appendix reaches its greatest anatomical development, macroscopic and microscopic, between the ages of ten and twenty-five, after which period it begins to involute. From this it appears that its greatest physiological activity is during the same period, and we know that it is at this timc excessively liable to pathological attacks.

All three coats of the appendix - the mucous, muscular, and serous-are directly continuous with those of the cæcum. The only one which is anatomically interesting is the peritoneal covering. The cæcum is almost completely surrounded by peritoneum, though it usually does not have a true mesocaecum. The appendix is practically always completely surrounded by peritoneum, and has a definite meso-appendix. Sometimes it seems to be extra-peritoneal, but this is, we believe, the result of pathological changes. Likewise, the curious contractions of the meso-appendix which render that 
organ tortuous, are, we believe, also the results of pathological change. And when we reflect that the appendix is essentially an organ in which bacteria luxuriate, it is exceedingly probable that lymphangitis of its mesentery, that may lead to subsequent organization of the inflammatory products and contractions, will be very common. The appendix takes origin from the caecum at the meeting point of the three longitudinal muscular bands, which point is $1 \frac{1}{2}$ to 2 inches from the ileocæcal junction.

Very many authors have attempted to estimate the frequency of the various positions of the appendix, some with anatomical names, others by the hands of a clock or by the points of a compass. But it is quite obvious that any such attempt will lead to cumbersome and useless classifications which may convey erroneous ideas. It is agreed that it lies internally to the long axis of the cæcum in by far the most numerous instances, and this is probably the case in about seventy-five per cent. In this position it may either lie just under the mesentery of the ileum or downwards in the pelvis. The frequency of the latter position has been very variously estimated, but the probable truth is that the appendix, wholly or in part, lies within the pelvis in more than half of these cases. It is underneath the caput coli in about fifteen to twenty per cent., and in the remainder lies in the iliac fossa, or external to the cæcum. The practical points which this anatomical disposition of the appendix indicate are:-

With the internal position-

1. The great liability of the small bowels and pelvis to be infected, and of that infection (peritonitis) to become general.

2. The early appearance of an inflammatory tumour due to the formation of adhesions of the small bowel and omentum.

3 . The extreme value of rectal examinations.

4. The liability of the adhæsions of the small intestines to cause, in the future, pain and perhaps intestinal obstruction. 


\section{SURGERY OF THE APPENDIX}

5. These cases are fraught with the most danger, and are by far the most common.

Behind the cæcum-

1. This is a fairly safe place, and from adhesions a localized abscess only is likely to arise.

2. An abscess in this situation may give rise to little or no physical signs, and is a danger in that a surgeon, thinking that he is removing an appendix in the quiescent stage, may not see the pus, and so foul the peritoneum. Often, too, the pus is present in small amount.

In the iliac fossa-

1. This is a fairly safe position, being probably intermediate between the internal and retrocæcal positions.

External to the cæcum-

1. This is the rarest and safest position in which the appendix is found.

The mucous membrane is like to that of the cæcum in containing similar glands, but differs in that it has more lymphoid tissue. The opening of the appendix into the cæcum is sometimes guarded by a fold of mucous membrane, called, after its describer, the valve of Gerlach. Sir Frederick Treves denied the existence of such a valve, but most authors have found it. Its existence depends really on an obliquity in the appendiculocæcal junction, and is of purely physical origin. Hence some have failed to see it, whilst others have found it frequently. As the oblique insertion of the appendix into the cæcum is in all probability almost always of pathological origin, the presence of the valve of Gerlach becomes of some slight importance in that it may obstruct the discharge of appendicular secretions and so bottle up the micro-organisms which are in its lumen.

A double fold of peritoneum attaches the appendix to the iliac fossa, or more usually the cæcum. Apparently this extends more nearly to the tip in infants than in adults, in whom the last half inch is destitute of mesentery and direct blood 


\section{PERITONEAL FOSS $Æ$}

supply, and also, not unnaturally, is the most common part to slough.

The vascular supply is derived from the ileocolic or the right colic branches of the superior mesenteric artery, or a communicating vessel between these two. The artery to the appendix runs in the free edge of the meso-appendix, but in other and pathological states numerous and spouting vessels are to be found near the attached border of the meso-appendix.

The nerves are derived from the superior mesenteric plexus of the sympathetic which also supplies the small intestine, so accounting for the fact that patients refer the pain to the umbilicus before a definite sign of a local peritonitis has appeared.

The lymphatics of the appendix deserve more attention than they have hitherto received. Every appendicitis is intimately connected with the presence of micro-organisms, and therefore there will be lymphatic infection. The lymphatics run in the mesentery to a gland at the ileocæcal junction, and thence to the mesenteric glands. Inflammation of the lymphatics of the mesentery is probably responsible for the twists and curves that the appendix may undergo. Lockwood has shown that in the appendiculo-ovarian ligament the lymphatics of the appendix communicate with those of the ovary, broad ligament, etc.

Some lymphatic glands situated along the inner border of the ascending colon seem also to be connected with the appendicular lymphatics.

\section{Peritoneal Fossce.}

The nomenclature of the various peritoneal fossæ round the cæcum has been rendered most confusing by the various additions of each observer. It will be desirable to adopt a clear, precise and easily remembered classification even at the sacrifice of small items of anatomical accuracy. There are three fossæ of importance round the ileocæcal region, the first 


\section{SURGERY OF THE APPENDIX}

of which is situated in the angle between the ileum and ascending colon, or a vascular trunk there. The name for this is the ileocolic fossa. The appendix is practically never in this fossa. The second fossa is found in the angle between the ileum and cæcum, its name being the ileocæcal fossa. The third fossa lies behind the cæcum, the retrocæcal fossa, and is either between the layers of the meso-cæcum or is bounded laterally by peritoneal folds. The latter is the more common.

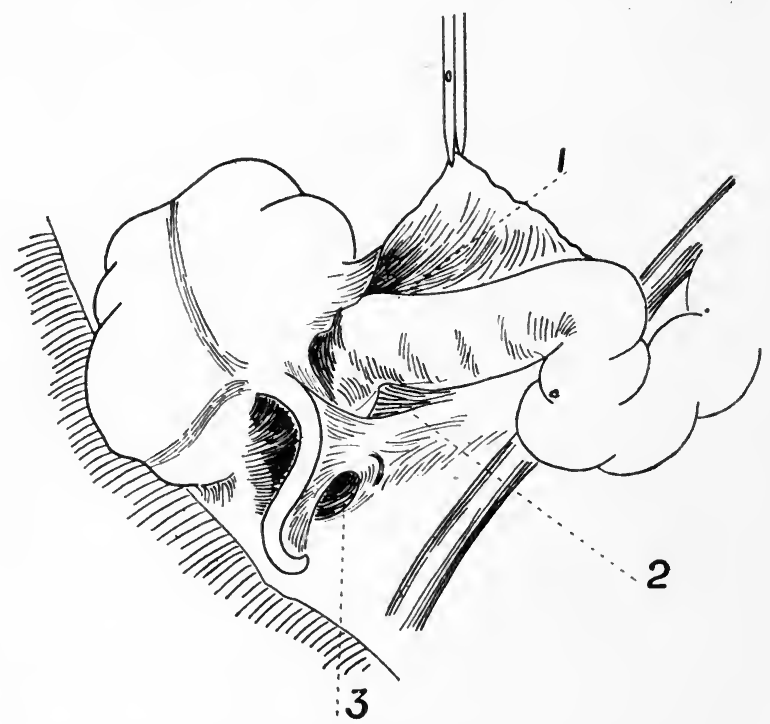

Fig. 1. The Peritoneal Foss about the Appendix. 1, Ileocolic. 2, Ileocæcal. 3, Retrocæcal.

The ileocæcal and retrocæcal fossæ can only be seen on raising the cæcum, and both are probably in more frequent relation with the appendix, wholly or in part, than is generally supposed. The practical importance of the fossæ is indicated by the following points-

1. The appendix in one of these fossæ when inflamed may give rise to a small, circumscribed abscess which will give but little in the way of physical signs. From this may arise frequently 


\section{SURFACE MARKINGS}

recurring attacks of localized inflammation, or even general peritonitis if the abscess is not opened and drained ; or, again, if an operator fails to notice its presence, he may foul the peritoneum, and so set up a general peritonitis.

2. Several surgeons have discovered an appendix by examining the fossæ, which otherwise they had failed to find. If an appendix becomes chronically inflamed in one of these fossæ, it may become sheeted over by adhesions, giving the impression that it is retroperitoneal.

3. Though the appendix is not likely to be strangulated in one of these fossæ, the small intestine may be. ${ }^{1}$

\section{Surface Markings.}

It is not possible to map out with exactitude on the surface of the abdomen so variable an organ as the appendix. The best known of the landmarks is that of McBurney, which is supposed to mark the origin of the appendix from the cæcum and the most tender point during an attack of appendicitis. This point is about $1 \frac{1}{2}$ to 2 inches from the anterior superior spine of the ilium along a line joining that point with the umbilicus. Roughly, it is situated about the junction of the outer and middle thirds of the same line. Clado has given a far less known, but more accurate method, by means of which the base of the appendix is localized, by a line joining the two anterior superior spines, at the point of its intersection with the outer edge of the right rectus. Clado's point is below McBurney's, and we consider it the more accurate. Mr. Addison has established the relation of the appendix to the abdominal wall, but by a more elaborate method. Prof. Cunningham localizes the orifice of the appendix on the surface "by placing the finger upon the right Poupart line one inch below the intertubercular plane." That is to

1 Mansell Moullin, Lancet, 1889, vol. i. p. 897. 


\section{SURGERY OF THE APPENDIX}

say, one inch below the point of intersection of a line joining the tubercles on the crests of the ilia with another drawn vertically up from the middle of Poupart's ligament.

All these points are of very doubtful clinical value, and Cunningham's seems the most accurate, though doubtless the others are serviceable with particular dispositions of the organ. Little or no reliance should be placed on these signs, for it is frequent for the pain to be referred to the flank, umbilical or gall bladder region. And if a rectal or vaginal examination is made in regular routine, it will be found that the tender point is by far the most common high up on the right wall of the pelvis, this pain being frequently more marked than that elicited by abdominal examination.

The great importance of this rectal examination is emphasized by the fact that the ordinary anatomical situation of the appendix is internal to the cæcum, in which position it is frequently partly in relation to the pelvis.

A few words must be added with regard to the anatomy of the abdominal wall over the appendix region. Anatomists get a very erroneous idea from the condition of the rectus muscle as examined by them. Weeks or months have elapsed since death, and the parts are displaced by dissection, shrunken by the coagulation of their albumens, and perhaps desiccated in the process of examination. Hence the outer edge of the rectus is usually figured about half way, or a little outside that point, between the anterior superior spine and the umbilicus. This is the situation of the linea semilunaris. At death the tonic contractions of the external and internal oblique muscles are lost, and the linea semilunaris will no longer be pulled outwards. In living subjects the linea semilunaris is situated at the junction of the middle and outer thirds of the line joining the anterior superior spine and the umbilicus. And very frequently it is further still from the middle line, even reaching to within an inch of the bony process. This con- 
stitutes a great difficulty in performing the fibre separating or paramuscular operation of McBurney, as it gives very little room. The best way of enlarging such a wound is by opening the rectus sheath, drawing the muscle inwards, and incising the posterior layer of the sheath in the line of the fibres of the internal oblique. A weak spot may easily occur at the point where the linea semilunaris has been divided. The incision advocated by one of us (Mr. Battle) is the most suitable for this situation in that it may be easily enlarged and is perfectly safe from the after development of a hernia. The incision is carried through these tendinous layers, first that of the external oblique, then the anterior, and lastly, the posterior layer of the rectus sheath. The rectus muscle has been retracted, and falls back in its place when the three layers are sewn up. The layer formed by the external oblique is not generally known, and may be shortly described.

Above the umbilicus the tendon of the external oblique is inserted into the linea semilunaris, but below that, into the anterior sheath of the rectus 1 to $1 \frac{1}{2}$ inches from that line.

\section{Absence of the Appendix.}

Even experienced surgeons find cases from time to time in which, after prolonged and possibly repeated search, they fail to find the appendix. Probably the organ is smoothly and surely sealed off in a cæcal fossa, and it may defy all except postmortem attempts to find it. Real absence of the appendix is a very rare thing. In 1900 , Picquand ${ }^{1}$ reported one case, and found nine others in the literature recorded by Morgagni, Haller, Santorini, Hunter, Anteirieth, Meckel, Massa, Tarensky, and Lafforgue. In the same year Professor Fawcett and Dr. Blatchford ${ }^{2}$ in the course of their examinations found another

1 Picquand, Bull : et Mêm. de la Soc. Anat. de Paris, 1900. 68, 2, p. 602 .

2 Fawcett, Journal of Anat. and Phys., vol. xxxv. p. 123 and lii. 


\section{SURGERY OF THE APPENDIX}

case which they reported to the Anatomical Society. Roberts ${ }^{1}$ recorded a case in which a cæcum in the sac of a right inguinal hernia showed no trace of an appendix, though he could freely examine the point of junction of the three muscular bands of the cæcum. Swan ${ }^{2}$ has given an account of a thoroughly authentic case of absence of the appendix. This brings the number of reported cases up to thirteen. Since the above was written Dillard ${ }^{3}$ has published a fourteenth case.

\section{The Minute Anatomy and Histology.}

The appendix consists of four coats, the mucous, the submucous, the muscular, and the serous, all of which are directly continuous with the corresponding tissues of the cæcum. The mucous membrane which lines the interior throughout, consists of a single layer of columnar epithelial cells set on a delicate basement membrane and of tubular glands of the ordinary large intestine type (Lieberkühn's glands), between which are situated nodules of lymphoid tissue. In some situations this lymphoid tissue is aggregated into masses or follicles. In perfectly healthy normal appendices a thin muscularis mucosæ can be seen surrounding the glandular mucous membrane. It cannot be differentiated into layers like that of the other parts of the intestine, and is very commonly wanting where the lymphoid masses or follicles are situated. Outside the muscularis mucosæ is the submucous tissue, which consists of delicate connective tissue, vessels, lymphatics, and nerves. In the healthy appendix it is well marked, but very frequently both it and the muscularis mucosæ have disappeared in part.

The muscular coat consists of an inner circular and an outer longitudinal set of fibres. The circular layer is thicker and

1 Roberts, Annals of Surgery, 1896, xxiii.

2 Swan, Trans. of Path. Soc. Philadelphia, 1898, xviii. 38-41.

3 Dillard, Virignia Medical Semi-monthly, Richmond, 1903-4, 418. viii. 
denser than the longitudinal. The longitudinal muscular fibres of the outer layer are very irregular in their distribution, which irregularity has been thought to represent an attempt at the formation of muscular bands, as is seen in the tæniæ musculares of the cæcum and colon. Where the mesentery of the appendix joins that organ, both muscular coats may be deficient in more than one spot, giving a freer communication than usual between the submucous and subperitoneal lymphatics. Such a space is called a hiatus muscularis. The serous coat consists of a layer of flattened epithelium resting on a layer of subperitoneal connective tissue of approximately the same thickness as that of the longitudinal muscular fibres. The subperitoneal tissue is continuous with that between the ' layers of the mesoappendix and that of the rest of the abdomen.

\section{THE PHYSIOLOGY OF THE APPENDIX}

It has long been taught that this organ was functionless, and that most probably in this lay its apparent incapability to resist the attacks of disease. That it represents the terminal portion of the cæcum that has become differentiated in development is undeniable. But that it is functionless is undoubtedly untrue. Like the rest of the intestinal mucous membrane, that of the appendix has numerous glandsLieberkühn's glands-but the secretion is more viscid than that of those of the small intestine owing to the presence of a larger amount of mucus. Apparently the fluid has a slight insignificant digestive action on all foods ; and it seems most probable that it is of more importance in aiding absorption than promoting digestion. In common with the other parts of the alimentary tract, the appendix may aid in this function. Very little; if any, contents of the cæcum can ever enter a normal appendix, whose peristaltic action would be opposed to it. Hence, any digestive action it has must be of the 


\section{SURGERY OF THE APPENDIX}

smallest. On the other hand, its absorptive action may be supposed to be a more important rôle.

In the small int etine the products of digestion are fluid, and are being continuously passed onwards by peristalsis. The cæcum is the first great "resting place" for these products since they have left the stomach. The large intestine, as its name implies, is of greater calibre than the small, and is more suited for the passing on of solid than of liquid fæces. The cæcum being rendered less mobile owing to its peritoneal attachments and having to work semi-fluid material against gravity, fæces have to remain in it until their consistence is such as allows of their passage. The change in consistence is much aided by the mucus of the glands of the large intestine. As a result, the cæcum of the child loses its tapering form and becomes larger by the development of pouches (see Fig. 2) between the three muscular bands. In bipeds, in whom the erect attitude has been assumed, gravity will be opposed to the passing upwards of the intestinal contents. During this period further quantities of digested material will be deposited in the cæcum and dilate that structure, possibly delaying the despatch of the first fæcal contribution. Hand in hand with these processes will go the fermentative changes of myriads of micro-organisms, to whom the temporary fæcal stagnation offers the opportunity of multiplication. Hence the far greater quantity of bacteria in the large than in the small intestine. The temporary delay in the passage of intestinal contents, the absence of a secretion like that of the stomach, the presence of large quantities of active micro-organisms and their products, point to the necessity for the development of a protective armament on the part of the host to counteract them. Hence we find the large development of lymphoid tissue in the terminal and most dependent part of the cæcum, i.e. the appendix, and its specialization, not degeneration. The need of the presence of this "intestinal tonsil" is apparent in the cæcal 


\section{DEVELOPMENT OF THE APPENDIX}

region. The comparative absence of lymphoid tissue in the rest of the large intestine is explicable in that in the cæcum the fæces have been brought up to the proper consistence to allow of their passage along the gut, and in consequence there is an absence of periods of "rest," and as the fæces become dryer and harder, there will be less fermentative action.

In this way may be seen the harmony between the condition of man and other animals, and the conclusions arrived at by Mr. R. J. A. Berry, of Edinburgh, ${ }^{1}$ may be reproduced-

1. Lymphoid tissue is the characteristic feature of the cæcal apex. The vermiform appendix of man is represented in the vertebrate kingdom by a mass of lymphoid tissue, situated most frequently at the cæcal apex.

2. As the vertebrate scale is ascended, this lymphoid tissue tends to be collected together into a specially differentiated portion of the intestinal canal, the vermiform appendix.

3. The vermiform appendix of man is not, therefore, a vestigial structure. On the contrary, it is a specialized part of the alimentary canal.

\section{DEVELOPMENT OF THE APPENDIX}

The development of this organ can be studied both in the individual and in the animal series. That of the human fœtus will be given first.

Up to the third month of intra-uterine life the diverticulum of the primitive intestine, which subsequently becomes the cæcum, is of similar calibre throughout. At this period the growth becomes unequal, and the terminal portion fails to increase in size at the same rate as the proximal. The inequality thus established becomes more and more marked as time goes on. At birth the terminal part of the cæcum, or the appendix as it is now called, forms a tapering prolongation of the cæcal diverticulum." It is not until later that a definite

1 Journal of Anat. and Phys., 1901.

2 Keith, Human Development and Morphology. 


\section{SURGERY OF THE APPENDIX}

line of demarcation is to be seen between the cæcum and appendix. The fœtal type of cæcum is, therefore, an inverted cone whose apex imperceptibly shades off into a prolongation of the appendix. It will now be seen that the appendix represents the terminal portion of the original cæcum.
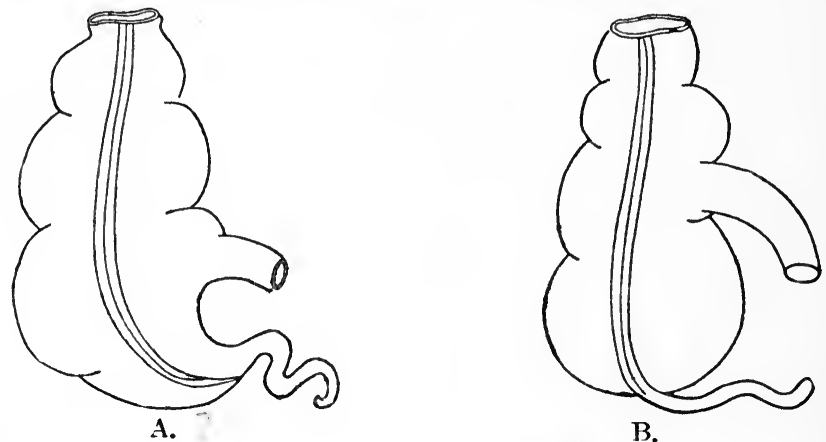

B.
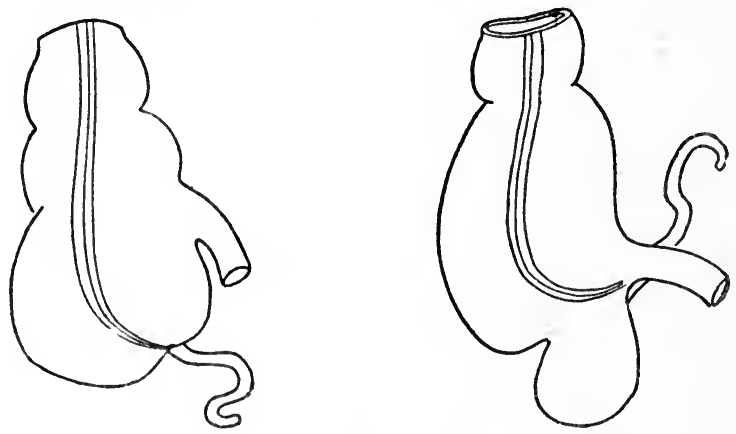

C.

D.

Fig. 2. Types of Development of the Cacum.

A, The Fœtal type. B. C. D., showing varying directions of development of the lower part.

Turning to the animal series for suggestions to aid in the explanation of this phenomenon, it is found that a distinctly marked appendix is only seen in man, anthropoid apes, lemurs, and the opossum. On the other hand, it is common to find a lymphoid structure at the distal portion of the cæcum, which undoubtedly represents the appendix. ${ }^{1}$ In the latter series

${ }^{1}$ Berry, Journal of Anat. and Phys., 1901. 


\section{DEVELOPMENT OF THE APPENDIX}

of examples the cæcum and its lymphoid appendage conform to the human foetal type. It is quite a later phase both in ontogeny and phyllogeny that the cæcum loses its conical form by the development of pouches (Fig. 2) between its three muscular bands. And it is by these means that the appendix becomes distinctly separated from it.

As age advances the appendix undergoes changes both in position and structure. In the fœtus it is situated along with the cæcum under the liver, and later it descends into the iliac fossa. Sometimes it remains in its fotal position, or it may be arrested at any part of its descent. An examination of the appendices for different decades shows that they are both relatively and absolutely longest and thickest between the ages of ten and thirty. The walls during this same period are stuffed with lymphoid nodules. After the latter age it begins to undergo a slow involution both in length and thickness. The lymphoid nodules disappear also, though more slowly and uncertainly. Berry ${ }^{1}$ looks upon obliteration of the lumen of the appendix as a physiological process occurring in middle and late life. The characteristic tissue of the appendix is the lymphoid tissue, a tissue that is known to undergo atrophy after a certain period of life, and it seems most probable that this atrophy goes hand in hand with the obliteration of the lumen. Ribbert ${ }^{2}$ also shares this view, and it is certainly more common to find obliteration in appendices from people between sixty and seventy than in earlier decades. In these cases it is impossible to decide how much is due to physiological changes of age, and, considering the great frequency of minor follicular appendicitis, how much is due to pathological conditions. Robert Morris ${ }^{3}$ has directed

1 Journal of Pathology and Bacteriology, 1895, p. 163. Anatomischer Anzeiger, 1895, Band x. 767.

${ }_{2}^{2}$ Ribbert, Virchow's Archives, 1893, p. 66.

3 Morris, Am. Journ. of Obstetrics and Gynaecology, 1901, p. 439. 


\section{SURGERY OF THE APPENDIX}

attention to the association of pain, intestinal and general symptoms with this involution. The change is certainly of a chronic inflammatory character such as would be expected to occur in nearly every one who has an appendix, and is the result of the continued irritative action of bacteria throughout years.

\section{Abnormalities.}

It may be of interest here to note several abnormalities. Rolleston ${ }^{1}$ has recorded a case in which the tip of the appendix pierced the plica vascularis, a fold of the peritoneum formed by the spermatic vessels, and underwent an attack of inflammation. Similar conditions arise where the appendix pierces the mesentery of the small gut, the cæcum, or its own. It seems very improbable that these arrangements can be congenital, especially considering the phenomenon of the descent of the cæcum. But rather does it appear that these various peritoneal holes are pathological in origin, and are the result, not the cause, of the primary attacks of appendicitis. Doubtless later they would cause recurrent attacks.

Dixon ${ }^{2}$ has reported a most interesting case that exemplifies the descent of the cæcum from under the liver of the fœtus. The appendix was removed postmortem from a woman of fifty years of age; it lay behind the cæcum and ascending colon with its body much attenuated and pulling on the cæcum, whilst a constricted bulbous end was in relation to the liver. The total length was sixteen centimetres. The probable explanation was that there was some fotal peritonitis that enclosed the tip of the appendix near the right kidney and liver. With descent of the cæcum the appendix was stretched into the condition in which it was found.

1 Rolleston, Journ. Anat. and Phys., 1897-8, p. 64.

2 Dixon, Ibid., 1896-7, xxxi. p. 442-5. 


\section{ABNORMALITIES}

Reference must also be made to the presence of the appendix on the left side of the abdomen. This is most common in children on account of the greater mobility of the cæcum at that age. Cases of left-sided appendicitis and appendicular abscess have also been described, and the presence of the appendix has been noted in umbilical, left inguinal, and left femoral hernias. 


\section{CHAPTER II}

Ætiology : The Pathology of Appendicitis: Chronic, Acute (Non-Suppurative; with Local Suppuration; with Peritonitis)-the Structure and Formation of Concretions-Leucocytosis-BacterioLOGY

\section{ÆTIOLOGY}

IT has been the custom to subdivide cases of inflammation of the Appendix in the St. Thomas' Hospital Reports into Appendicitis, Appendicitis with local abscess, and Appendicitis with general peritonitis. Consequently it is under these headings that statistics can be obtained, and as generally speaking only the more severe types of disease are brought to Hospital, the severity and frequency of complications may be regarded as a maximum.

During the years 1894-1901 inclusive, 525 cases were brought to the Hospital suffering from appendicitis of the apparently non-suppurative variety, 150 of the localized suppurative variety, and 108 with "general" peritonitis. Almost all with general peritonitis were in their first attack. Generally speaking, abscess occurred in later attacks, but a good number happened during the first.

Taking all the cases together about which details were ascertained, 70 per cent. were in their first attack, 20 in their second, and 10 in their third or more. The percentage of those which had a second or more attacks is therefore 30 . The mortality, however, was very different; that for the first attack was 25 , that for the second 7 , that for the third 2 . There has always been considerable difficulty in differentiating 


\section{ETIOLOGY}

\section{NUMBER OF}

\section{CASES.}

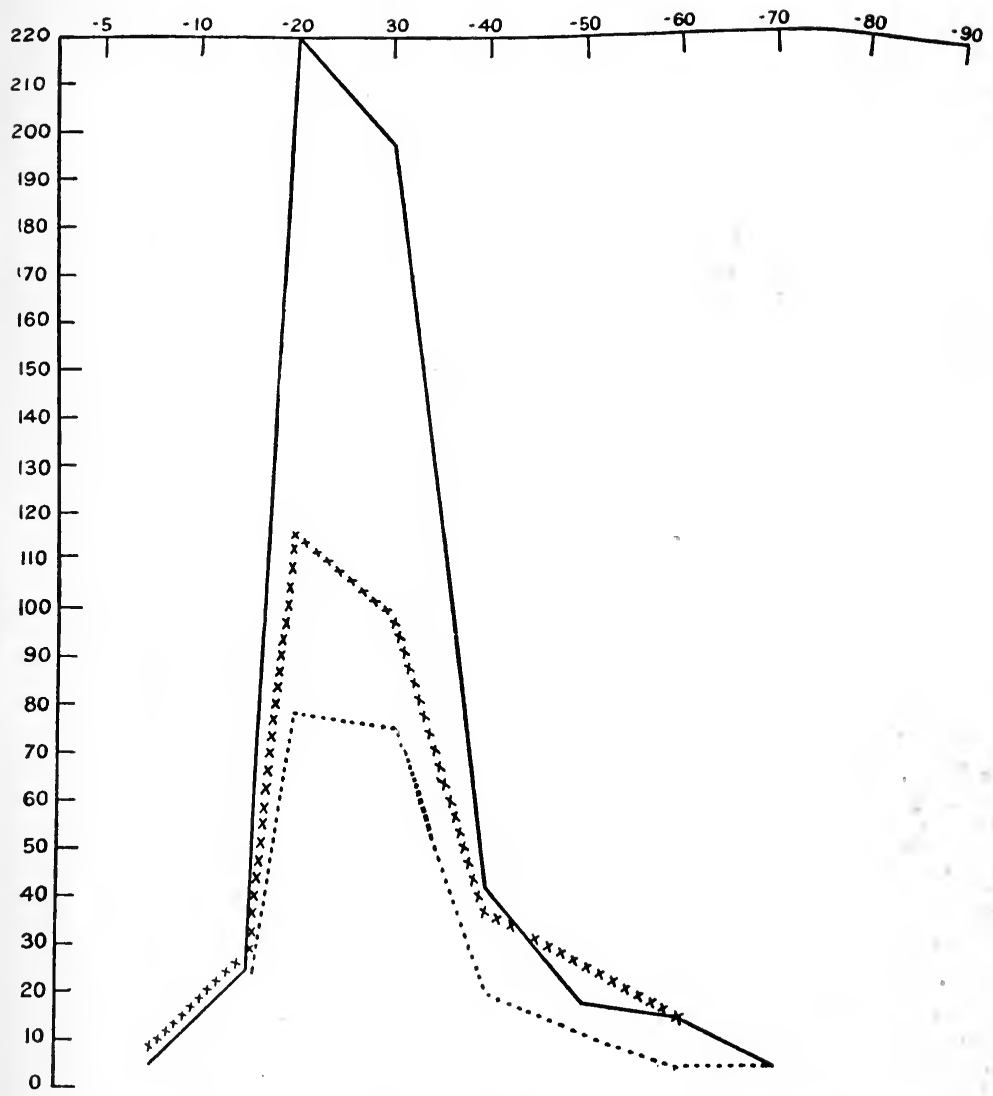

ACE INCIDENCE OF APPENDICITIS; WITHOUT SUPPURATION WITH LOCAL ABSCESS $x x x x x$, WITH DIFFUSE PERITONITIS.

Fic. 3. 


\section{SURGERY OF THE APPENDIX}

between cases of appendicitis that have had a second or further attacks, and those which have fresh outbreaks every few weeks, as to which are meant by the term "relapsing appendicitis." According to the St. Thomas' figures, one in every three will have another attack.

Appendicitis is rare in babies, although it has been described in a baby of seven weeks old. It is most common in all its forms between the ages of ten and twenty, then between twenty and thirty. Compared with the frequency of its incidence in these two decades of life, that in the first, fourth, fifth, sixth and seventh decades is insignificant. The oldest recorded sufferer from appendicitis was eighty. With regard to the two great complications, namely abscess and peritonitis, they are most common in the second decade, but in the third the latter is relatively more common than in the second.

After this the curves decline in equal ratio until at the seventh decade they become equal. Another and more important rule can be deduced. The relative proportion of cases in which suppuration takes place increases most markedly with age, until in the fifth and sixth decades the suppurative cases, abscess or peritonitis, are absolutely more frequent than the apparently non-suppurative cases. Under the age of ten, suppurative appendicitis in both its forms is absolutely more common than the non-suppurative variety.

With regard to sex, males are more frequently the subjects of appendicitis. The St. Thomas' figures for non-suppurative cases are three males to one female ; for local abscess, less than two males to one female; for peritonitis, rather over three males to one female. Interpreting these results in words it may be said that relatively, peritonitis is a little more common in men, but abscess is much more common in women.

Occupation and pregnancy seem to have little effect upon the incidence of appendicitis. Spring and autumn seem to be the 


\section{CHRONIC APPENDICITIS}

seasons when this disease is most evident. In fact it seems most frequent at the rise and fall of the leaf.

A number of the cases give some history of constipation or trouble with the bowels, and it is not possible to separate those cases in which the appendicitis may have been so brought on, from others in which the bowel trouble is secondary to a subacute appendicitis. It is certain that the two conditions may be related. The incidence of the attack is not unfrequently attributed to some transient dietetic indiscretion, or exposure to cold. Byrom Robinson has attributed the incidence in a large number of cases to the action of the psoas muscle. But to whatever cause it may be attributed the chief active agents in its production are the organisms in its lumen.

\section{PATHOLOGY}

To this subject a great deal of attention has been given; and it is consequently far from easy to be able to give a short, clear and concise account of it. Firstly, it will be best to consider a chronic form of appendicitis, whose existence and importance are as yet scarcely recognized clinically. Afterwards, the pathology of the more acute, and, therefore, clinically recognized attacks can be discussed.

\section{Chronic Appendicitis.}

It is a matter of more or less common knowledge that as years pass on changes are found in the appendices of people who have never had a clinically recognizable attack of appendicitis. How much these changes are physiological it is impossible to decide, and in this connection it is not so important, because, whatever they are, they will interfere with the normal performance of the work of the appendix, and so lead to evil results. One of the best known of the changes already mentioned is the disappearance of the lymphoid follicles that are so plentiful in early life. Is this disappearance pathological or 


\section{SURGERY OF THE APPENDIX}

physiological ? Yet if sections are cut of appendices removed from people over sixty, or even older, these follicles can often be found in abundance. The persistence in these cases may be due to individual variations in the rate of ageing, or perhaps more probably, they indicate that the disappearance is of more pathological than physiological import.

In dealing with the physiology of the appendix, it has been shown that the cæcum is the first great place of rest for the products of digestion after they have left the stomach, and that the appendix was a tube which served as an index to the chemical and bacteriological processes that occurred in the cæcum. One well-known property of lymphoid tissue is that its constituents are actively protective to the organism against foreign invaders. Situated in a blind tube in whose lumen are represented types of the organisms of the large bowel and their fermentations, the lymphoid follicles must have a great deal of work to do, more even than the tonsils to which the appendix has been likened. It is well-known that the tonsils undergo fibrosis or chronic inflammation without the occurrence of an acute attack. In a like manner do similar changes occur in the appendix, but unfortunately here there is a very marked difference. Fibrosis of the appendix interferes with the completeness of its peristaltic action, and as a result the tube will become incapable of emptying itself. By these means a vicious circle is established in that more pabulum remains within for the bacteria to flourish upon, and the more bacteria flourish, the more likely is it that the chronic inflammation will progress, and the organ become still more incapable of performing its own evacuation. Such a condition may be aptly called appendicular constipation. The inspissation of the contents will lead to the formation of an appendicular calculus or fæcal concretion, which may be likened to scybala elsewhere in the large intestine. It may thus be assumed that the presence of concretions indicate 


\section{CHRONIC APPENDICITIS}

the co-existence of pathological change. And their association with chronic appendicitis explains the fact that they are frequently found by the surgeon and the pathologist in cases that have never had a recognised attack of appendicitis.

The results of chronic appendicitis may be and are far more extensive than this. The fibrosis and its subsequent contraction leads to the disappearance of the lymphoid follicles and the further impairment of the muscular action. The appendix consequently becomes the inert breeding ground for bacteria, and if the products of their action escape into the cæcum the multiplication is continued there. The cæcum will be affected sooner or later by the continual escape into it of toxic material elaborated in the appendix, and as it is necessary that the fæces remain there until they are of suitable consistency to be passed on into the ascending colon, those products will become still further elaborated and a chronic typhlitis arise. Again there is the vicious circle in that the inflammatory condition will impair the contractile power of the cæcum, and so lead to further bacterial growth and further chronic inflammation. In this way a colitis may be established throughout the whole length of the large bowel. Such a condition can of course arise from changes in the large bowel alone, although in any case the appendix must be regarded as a most important factor, if not the originator. If the cæcum contracts adhesions its power of perfect evacuation will be still more impaired, and it will allow more easily of the production of an inflammatory condition such as the above.

It is then easy to see the association and interdependence of colitis, typhlitis, and appendicitis. Clinically these cases are extremely interesting and important, and doubtless many of the instances of dyspepsia, constipation, mucous colitis, abdominal discomfort, etc., are of this origin. In fact, chronic appendicitis is one of the most common of all diseases, but 


\section{SURGERY OF THE APPENDIX}

at present it is impossible to say in what number of cases it produces symptoms, and in what number removal of the organ will produce amelioration.

Acute Appendicitis.

Apparently Non-suppurative. All cases of appendicitis are probably of septic or bacterial origin, and there are no lines of distinction between the various elinical courses or pathological varieties. In dealing with chronic appendicitis, attention was drawn to the anatomical position and the physiological functions of the organ, and from these its pathology derived. And it was surmised that the inflammatory changes arose primarily in the lymphoid nodules, and were soon followed by chronic inflammatory conditions of the mucous membrane, cellular tissue, muscle, etc., leading to fibrosis of the organ. In the more acute cases, which are, therefore, easily recognizable clinically, the origin is probably more complex. And it will be easier to consider the apparently non-suppurative or resolving cases first, and the obviously suppurative second.

The first recognizable attack arises either in a normal appendix, or, as is probably more common, in one that is already the subject of chronic appendicitis and may contain concretions and so forth. When the latter conditions are present there is a tendency for the case to become of the suppurative variety. The distinction of apparently and obviously suppurative classes is convenient, though quite arbitrary, and both have a similar septic origin.

If the acutely inflamed appendices are removed very early after the onset of the attack, three varieties at least can be recognized-

1. When the mucous membrane is uniformly red and the changes are confined to that part, the peritoneal surfaces showing little alteration. 


\section{ACUTE APPENDICITIS}

2. When the mucous membrane is red, but the inflamed lymphoid follicles stand out very prominently.

3. When the inflammation is more in the submucous cellular tissue than elsewhere.

The first of these may be an endo-appendicitis comparable to the enteritis elsewhere; but differs so much from it in its clinical symptoms that one turns for another explanation. The likeness of these cases, both clinically and pathologically, to erysipelas had led one to regard many attacks of acute appendicitis as examples of capillary lymphangitis of the mucous membrane, or erysipelas, just as cutaneous erysipelas is a capillary lymphangitis of the skin. When it is remembered that the lumen of the appendix contains cultures of the many and various organisms of the cæcum, it cannot be surprising if such attacks occur frequently.

The second condition in all probability arises in the lymphoid follicles-i.e. a folliculitis-and is comparable to an acute tonsilitis. And it seems probable that far the greater number of so-called catarrhal cases would fall into this category.

The third subdivision is a definite cellulitis of the appendix, that is to say it is a lymphangitis of the submucous and cellular tissue which may rapidly spread to the mesentery, and by causing secondary arteritis and phlebitis, i.e. angeitis, followed by thrombosis, results in gangrene of part of the appendix.

In the above the case may have been primarily an acute appendicitis, but quite commonly the acute attack occurs on the top of chronic inflammation. It is especially prone to do this on account of the resulting facilities for bacterial growth and action, the presence of retained secretion and concretions and so forth. Although these processes arise in the action of micro-organisms, they are not always obviously suppurative. Doubtless pus and small abscesses are more often present than is thought and these discharge into the 


\section{SURGERY OF THE APPENDIX}

bowel, the pus being in small quantity and passed unnoticed per rectum.

The results of an attack of appendicitis are many, and are of sufficient importance to be considered in detail. One of the most important is the effect of the condition left behind as regards the probability of the occurrence of further attacks. As this question is bound up with that of the gross changes that may result, these will be considered first, and so lead up to the larger question.

In constructing this catalogue, no attempt has been made to separate the attacks which have originated in the various ways suggested, but only those of a clinically recognizable type are detailed.

Resolution. As the onset of the attack was brought about by an exaggeration of what may be termed the natural septic condition of the contents of the appendix, those contents will tend to prevent resolution occurring and the complete disappearance of the inflammatory changes. But in cases that have been of the folliculitis or the erysipelatous character the acute conditions may subside and leave the peritoneum and other structures unaffected. In this way those cases, which every surgeon is familiar with, in which after one or more definite attacks yet a normal looking appendix without adhæsion is found, become intelligible. This would especially be likely to be the case with erysipelatous cases because, as is known, there is little tendency for cutaneous erysipelas to affect the deeper structures. And along with this there may be constitutional symptoms of all degrees of severity.

Chronic Appendicitis. The organisms naturally present in the appendix will tend to keep alive the inflammatory process. The natural result is that an attack of appendicitis will in all probability leave that structure in such a condition as will impair the normal performance of its functions. Fibrosis 
is always the great feature of chronic inflammation, and will hinder the peristalsis of the appendix.

Fibrosis. Sclerosis of the appendix in part or generally is the result of a process of chronic inflammation which will render that organ stiffer and less flexible than normal. This fibrosis will make it impossible for the appendix to perfectly evacuate its contents, and will therefore be a great predisposing condition for the further growth of bacteria; and in such a way may bring about a further attack, or a chronic toxæmic condition. The fibrosis may be general or local. In the former case one of two conditions may be produced-

1. A tendency to general or partial diminution of the size of the lumen.

2. Or, the walls being stiffened, the appendix may be held open, perhaps in its dilated condition, i.e. appendicectasis.

Ulceration. Should the irritant that is causing the inflammation of the appendix continue to act beyond the stage of mere multiplication of the connective tissue cells, the process cannot cease, but a further step must be taken; and the cells nearest to the irritant will undergo a process of molecular death, commonly called ulceration or suppuration. Such a condition belongs really to the suppurative class of appendicitis, but is far more common than is obvious suppuration. In the apparently non-suppurative cases the discharge is carried off by the bowel and is unnoticed. The evacuation of the abscess may take place through the perforation which has caused it.

Stricture. This exists in more than one variety and may be divided into two great classes, the organic, when there is gross change in the appendix narrowing its lumen, and the functional, in which the appendix itself is not grossly diseased.

In the first class the stricture is most frequently due to ulceration of the mucous membrane which has subsequently healed and the scar contracted. In a less common variety the stricture is due to a cellulitis of a segment of the 


\section{SURGERY OF THE APPENDIX}

appendix, where the mucous membrane is not destroyed. In this case the fibrous tissue is between the mucous membrane and the peritoneum.

In a third variety the narrowing of the appendix may be due to the contraction of peritoneal adhesions, or to the pressure of pathological processes (e.g. carcinoma of cæcum), which have secondarily involved the appendix.

The cases in which the lumen of the appendix is narrowed in one place, though the organ is healthy, fall into two great classes. In the first the appendix is bent or kinked, which functionally narrows its lumen, though it is not apparently diseased. Oblique insertion of the appendix into the cæcum also comes under this head. In the second class the appendix is pressed on from without, and its contents dammed up, as in carcinoma of the cæcum.

Mucoceles and Cysts. These arise from the damming up of the secretion in the appendix behind a stricture. They are commonly seen in the bulbous ends of strictured appendices. They may hold as much as four ounces.

Empyema. These are less common and result from the retention of pus behind a stricture. The pus formation which occurs is due to a later attack (or chronic ulceration), than that which gave rise to the stricture. Usually the pus can escape into the cæcum.

Appendicitis Obliterans. Obliteration of the cavity of the appendix is said to be the normal result of increase of age. Such change is in the highest probability the result of pathological processes. Chronic appendicitis leads to general sclerosis of the tube and by contraction of the fibrous tissue to diminution of its calibre. If by a process of ulceration the mucous membrane has been destroyed, two layers of granulations will be firmly apposed by the contracting fibrous tissue. If the débris arising from such a process be discharged into the cæcum complete or partial obliteration of the appendix may 
take place in a case of apparently non-suppurative inflammation. The contraction of fibrous tissue in the submucous laver may also lead to impairment of the nutrition of the epithelium, and its consequent shedding.

Appendicular Myositis. This is seen in the degeneration of the muscular coats, general and local, and their replacement by fibrous tissue. A name has been given to it because it emphasises the fact that appendicitis leads to inability of the organ to contract so as to completely expel its contents.

Appendicular Peritonitis (or Peri-appendicitis). The adhæsions that surround an inflamed appendix arise from this. How often this occurs it is impossible to say, as adhæsions are known to clear up in a truly marvellous manner.

Lymphangitis and Inflammation of the Mesenteriolum. This is one of the most common and least appreciated complications of appendicitis. The enlarged lymphatics run in the mesenteriolum and can be felt as cords of thickening. Lymphangitis is naturally associated in degree with all forms of appendicitis owing to its septic origin, and the subsequent contractions of the inflammatory products in the mesentery account for the curious twists which kink the appendix, so causing a functional stricture.

- Lymphadenitis. Infection of the lymphatic glands must be common in appendicitis, and if they are felt during the operation in acute cases they will be found to be enlarged and succulent, especially the one situated at the ileocecal junction. Yet suppuration is uncommon, and generally this is so for all the abdominal glands. Septic processes are exceedingly common in the abdomen, and yet it is very uncommon to find suppurating glands. It has been shown that bacteria. taken by the mouth do not multiply much until the ileocæcal junction is reached. But the lymphatic glands that drain the alimentary tract will be more or less inured to the products of bacteria, and especially so those connected with the large 


\section{SURGERY OF THE APPENDIX}

bowel. This may account for the rarity of suppuration in them.

The pathological results of a "non-suppurative" attack of lymphangitis have been briefly reviewed, and the question arises as to in what way the conditions left behind may affect the possibility and probability of future attacks. If there had been only a mere shedding of some cells from the mucous membrane, the organ is left in a precisely similar condition as regards a further attack as it was before. These very mild attacks will have been scarcely if at all noticed by the patient. In attacks of appendicular erysipelas the organ may similarly be but little changed. In fibrosis the chief effect will be that the appendicular peristalsis will be interfered with, and the organ unable to effectively expel its contents. Thus further opportunities are left for fermentation and the formation of concretions. Ulceration will doubtless lead to the formation of a stricture which will cause retention of some of the contents and most probably organisms behind it, and either a mucocele or an empyema may result. The appendicular myositis will lead to imperfect peristalsis, and a condition like that resulting from fibrosis. Periappendiceal adhæsions about the appendix may lead either to this or to those of stricture. The lymphangitis, if it does not properly resolve, will produce irregular contraction and crinkling of the mesentery with consequent kinking and tortuosity of the appendix itself. Any result of lymphadenitis is unlikely to affect the appendix. It will thus be seen that it is most unlikely that one attack of appendicitis will confer protection against a future attack, but rather that further trouble may be expected. The frequency of recurrence has been estimated variously at from 16 to 100 per cent. ${ }^{1}$ Appendicular colic will not usually be a primary affection, but the result of the formation of a concretion, stricture, kinking (relative stricture of the appendix), etc.

Pathology of Suppurative Appendicitis. In considering the

${ }^{1}$ pp. 198 and 199. 


\section{SUPPURATIVE APPENDICITIS}

pathology of the forms of suppurative appendicitis it may be again pointed out that only the obviously suppurative conditions are meant, such as abscess or peritonitis. In many cases abscesses discharge into the lumen of the appendix itself, less commonly into the cæcum or small gut. The pus in such cases, will be passed in a more or less altered condition per rectum unnoticed. The particular shape which the process takes in different cases depends upon a number of factors of which may be mentioned the anatomical situation of the appendix, the presence or absence of adhæsions, the virulence of the infection and the site of the gangrene, or perforation, etc. The channels along which the infection progresses are of importance for the understanding of the different manifestations which may be exhibited. But before giving an account of these it may be of interest to recount the steps by means of : which the perforation or gangrene of the appendix arises.

The inflammatory process begins in the mucous membrane and spreads thence to the submucous tissue. Here it follows in the main two courses, rimely along the lymphatics and towards its best blood-supply. As a natural consequence sooner or later a set of vessels will be reached and their lumens narrowed. by the pressure of the inflammatory exudation. This will be speedily followed by an inflammation of both veins and arteries, a general angeitis, and the final stoppage of the bloodstreams in them by thrombosis. The organisms then act on tissues deprived of their blood-supply, and speedily cause gangrene. The process should be called an acute infective necrosis. In the majority of cases the terminal half inch or so of the appendix is involved, and for two reasons. First and most important, the tip of the appendix is the part which peristalsis is the least competent to empty, and organisms will collect and thrive there. Secondly, it is the part that usually has no mesentery, and so, no direct blood-supply. Hence it will necrose when the vessels in the distal part of the mesentery 


\section{SURGERY OF THE APPENDIX}

become affected. If perforation occurs elsewhere it will usually be associated with the presence of a fæcal concretion at that spot, with its additional initiative action and that of the organisms which congregate upon it. It is unusual to find a concretion in the distal segment of the appendix, though one can be not infrequently easily displaced there.

The most frequent position for the appendix to be in is internal to the long axis of the cæcum, where it may be partly or wholly in relation to the pelvis. Probably therefore the first structures to be involved in the inflammatory process are the adjacent coils of small intestine. This is very important, because general peritonitis may involve a very large area of the peritoneum covering the coils in a very short time, any damage to the small intestine causes greater shock and more severe symptoms than in other regions, the area injured may be at an early date very large, several blocks in the intestine may be established by portions of several coils becoming paralysed by toxic inflammatory action, therefore abdominal distension may be great and early. So it may be seen how rapidly peritonitis of appendicular origin becomes serious and fatal unless immediate operation is proceeded with.

After the small intestine, the omentum forms barriers of adhæsions more easily than the other structures in the abdomen.

As the appendix is situated always below the level of the mesentery of the small bowel, the tendency of the infection is to spread to the parts of the abdomen downwards towards the pelvis rather than to the abdomen above. This and the fact that the appendix is frequently in a more or less degree in the pelvis indicates strongly the need and importance of rectal examinations.

If by chance the pelvis is first affected the rapidity of spread of the infection along the small intestine seems less, and the coils tend to adhere and roof in the purulent collection. As a result the 


\section{SUPPURATIVE APPENDICITIS}

process may spread along the rectum to the left iliac fossa, causing either a local abscess or one of the most interesting phenomena in this disease, namely, a left-sided peritonitis with left-sided pain, tenderness, and distension producing marked asymmetry of the abdomen and so forth.

Every operator in cases of general peritonitis knows how often in early cases the most affected coils lie in the pelvis, where they have apparently not had time to subside on account of their distension with fluid. Abscesses frequently track towards the pelvis so that they seem to be incompletely shut off in that direction. By this tracking is explained also the occurrence of pelvic abscesses, abscesses under the left rectus, in the left iliac fossa, left loin, and even under or over the spleen or in front of the left kidney. The occurrence of such abscesses after the appendix symptoms have subsided gave rise to the idea of a remote or distant peritonitis as their cause.

If the appendix lies in a peritoneal fossa or under the cæcum the abscess is very likely to be walled in by the omentum and small bowel. It is only when the appendix lies in a specially superficial condition as in the iliac fossa, or in front or along the outer surface of the cæcum, or the abscess is a large one whose drainage has been neglected, that the infection spreads along the outer side of the ascending colon, to the subhepatic, lateral hepatic, suprahepatic or subdiaphragmatic spaces. Secondarily from this channel of infection along the inner side of the colon, and the hollow in front of the right kidney become infected. But it is distinctly uncommon for infection to spread primarily along the inner wall of the cæcum, crossing the termination of the ileum en route. By this means explanation is afforded for the right lumbar, subhepatic, subdiaphragmatic and prerenal collections of pus. 


\section{SURGERY OF THE APPENDIX}

The Forms of Peritonitis Associated with Appendicitis. Inflammation of the appendix will practically be always associated with inflammation of the peritoneum round it. It is conceivable that though all inflammations of the appendix are of septic origin, yet a simple inflammation may precede that of the septic in its progress. Thus it seems possible that there may be an acute and chronic simple peritonitis. The former will be considered first.

The existence of an acute simple peritonitis which is associated with effusion is a certain clinical fact, yet considering the splendid culture medium that peritoneal fluid offers for organisms, it seems improbable that the peritonitis can remain simple for long. On the other hand, its presence is vouched for by many eminent and reputable observers. And those who have tried know that one does fail sometimes to obtain cultures from the early, and sometimes copious, effusion due to appendicitis. It has also been suggested that in localised collections of fluid the originating organism may have died out, or the peritonitis may have been tubercular, rheumatic, or gouty in origin. These latter remarks apply most forcibly to chronic simple peritonitis with effusion whose existence is well authenticated by continental surgeons. Far the most common is the chronic peritonitis that leads to the formation of adhæsions. It is possible, or rather probable, that in many cases the primary onset of the peritonitis was accompanied by some local effusion. But considering the capabilities of the peritoneum to remove and to deal with both fluid and organisms, it would be unwise to hazard a guess as to the character of this fluid, simple or septic.

Purulent peritonitis may be diffuse or localised, acute or chronic. The spread of the infection, its various opportunities and paths have been already discussed. The most important agents in diminishing the spread of a collection of pus are firstly the coils of small intestine, and secondly, the omentum. From the common position of the appendix these structures 


\section{SUPPURATIVE APPENDICITIS}

together form the greater part of the abscess wall, and are matted by an adhæsive peritonitis. These abscesses have been located in five chief situations, under the right rectus, in front of the cæcum, in the right loin, in the right iliac fossa, and in the pelvis.

Between the situation of the abscess and that of the appendix there must be some causal relationship. But it is very uncertain work to attempt to diagnose the position of the appendix from that of the abscess. The position of the appendix, in far the majority, is internal to the cæcum. Yet clinical experience soon teaches one that that is not so commonly the situation of an abscess. And it appears that persons whose appendices do not lie internal to the cæcum have amongst them a higher percentage of localised abscesses than their more normal brethren. The possible reason for this lies firstly, in that with the outward direction of the appendix its insertion into the cæcum is apt to be more oblique and valvular than in the more common disposition ; and, secondly, if suppuration occurs, as it seems more likely to with the external or retrocæcal arrangement, a localised abscess will in all probability result. Consequently, although people with externally directed appendices are more liable to appendicitis and abscess, they enjoy some compensatory immunity from general peritonitis.

The fate of the abscess is not of such importance nowadays with early surgical intervention as it was formerly. Still of abscesses left to themselves as collected from the literature and hospital reports the following table gives approximately the directions in which they are likely to burst :-

* Through the abdominal wall

* Into the cæcum

,, ,, peritoneal cavity

, , pleural cavity

, ,, ascending colon

,, , bladder

The rest into the ileurn, vagina, uterus, etc.

45 per cent.

36 ,,

5 , ,

4,

2 ,

3 ,

2 , 


\section{SURGERY OF THE APPENDIX}

Of this table the first two, marked with an asterisk, are undoubtedly too low; and there are omitted, as there must be, the abscesses which discharge viâ the appendix itself or the small bowel, the opening in which subsequently heals. (The above statistics were compiled by Dr. A. O. J. Kelly, and are published in Dr. Deavers' Treatise on Appendicitis, 1900, p. 119.)

The pathology of diffuse suppurative peritonitis is a subject that need not occupy much space. Diffuse suppurative peritonitis generally results in patients in whom the appendix is situated internally to the cæcum. The process starts as a local one, though it is not localised. It spreads to the peritoneum covering the small intestines, and so involves a far larger extent of peritoneum in a given time than is the case with other positions of the organ. The involvement of so much highly sensitive peritoneum with great power of absorption, the impairment of intestinal movements in several segments of the bowel, etc., indicates how severe and fatal a toxæmia this complication must produce unless timely intervention occurs. Clinically there are many types: from the slow insidious serofibrinous form to the most fulminating and hæmorrhagic varieties. The intermediate cases between these two extreme classes are the most common.

Peritonitis may become diffuse or general as there are no limits put to it. But one of the most serious forms which exists is when the inflammation arises through the leaking of an imperfectly circumscribed abscess. The diffusion of septic material over so large an absorptive surface may lead to the most profound toxæmia; so that some patients succumb while there is yet comparatively little peritonitis, yet others exhibit the greatest tolerance. The coils of intestine become infected, distended, and full of fluid, which stagnates owing to the toxic paralysis of the bowel, the fluid within the gut teems with organisms, and soon the patient is being poisoned 
from within as well as from without the bowel. In this lies the rationale of the treatment of these cases by purgation and infusion, to dilute the poison and replace the fluids lost viâ the bowel.

The purulent fluid in the peritoneum varies considerably in colour, amount, and consistence. In many cases it is copious, watery and malodorous; whilst in others, apparently of slightly better prognosis, it is thicker and more creamy, though perhaps very offensive; yet sometimes it is thin and of a brownish colour, due to some hæmorrhage into it.

\section{THE RÔLE OF CONCRETIONS IN APPENDICITIS.}

The earliest observers who detected concretions in the appendix regarded them from their shape and appearance as various foreign bodies, such as cherry-stones, orange-pips, grape-stones, etc. During the last decade it has been proved that the presence of such substances is in reality distinctly uncommon, and that appendicular concretions are really inspissated fæcal matter. In experimentally isolated loops of intestine, or in ligatured appendices, after a time the contents are found to be pultaceous and fæcal in appearance. It is the inspissation of such materials secreted by the appendix which leads to the formation of appendicular calculi. Normally it is very doubtful if any of the more solid contents of the cæcum enter the appendix at all. And it seems probable that the authentic accounts of the occurrence of hairs, pins, seed, etc., in the appendix may be in reality secondary to a previous inflammation, which has lessened its power of muscular contraction.

It is well known that such lymphoid structures as the tonsil may become tough and fibrous without the occurrence of any acute inflammatory attack. So will it be to some extent with the appendix, and the results of such chronic inflammatory change will be, at least, impairment of the completeness of peristaltic action. As a result, cæcal contents may possibly 


\section{SURGERY OF THE APPENDIX}

enter the interior of the appendix from above, and the material already in that organ will be imperfectly expelled. This may be aided or produced by the anatomical way in which the appendix is inserted into, or originates from the cæcum. And the common state of affairs to find is a functional stenosis of the lumen such as that arising from kinking or obliquity of the appendix, i.e. not an intrinsic, but an extrinsic stricture. The retained appendicular secretion will later become inspissated. The presence of concretions or calculi may therefore be regarded as evidence of some pathological change. They represent in the appendix the acme of constipation, such as is found elsewhere, and are formed by a process which may be called appendicular constipation.

Structurally, these calculi sometimes have for a nucleus some foreign body, but much more commonly a harder fæcal mass, which grows by the inspissation of mucus, etc., deposited on it by the tubular glands. It is very probable that many of these concretions are formed as the result of the action of micro-organisms as in the case of gall stones. This outer coating of moister fresher material offers to the organisms which are present, or which gain access from the cæcum, a suitable nidus for their growth. The presence of a concretion is therefore a danger, and a sure indication of the advisability for the removal of the organ. Fæcal concretions seem equally common in the two sexes, and what is significant is that they are most common between the ages of fifteen and thirty, i.e. the period of the greatest frequency of appendicitis, especially in its more severe forms.

The subsequent history of a calculus depends upon its bacteriology, present and future. It may remain quiet, and this is especially so in older people in whom the lymphatic nodules may atrophy, and the glandular secretion become more scanty. The concretion then becomes drier and harder, 
and unsuitable to the growth of bacteria. Again, an appendix whose secretion has long been retained undergoes physiological and anatomical atrophy. Thus the surgeon may discover, whilst exploring the abdomen of an elderly patient, an appendix with thin translucent walls containing one or more black hard calculi that shine through the tissues. These are quiescent and may be left alone.

Sir F. Treves has reported an instance of some interest as showing the slight amount of irritation sometimes caused by these concretions, in which one was found lying free in the peritoneal cavity, having escaped from the appendix some long time before the operation during which it was discovered.

On the other hand, it is very important to appreciate that the association of concretions, especially with the more severe forms of appendicitis, is an undoubted fact. To illustrate this the figures given by Dr. Deaver may be quoted :-

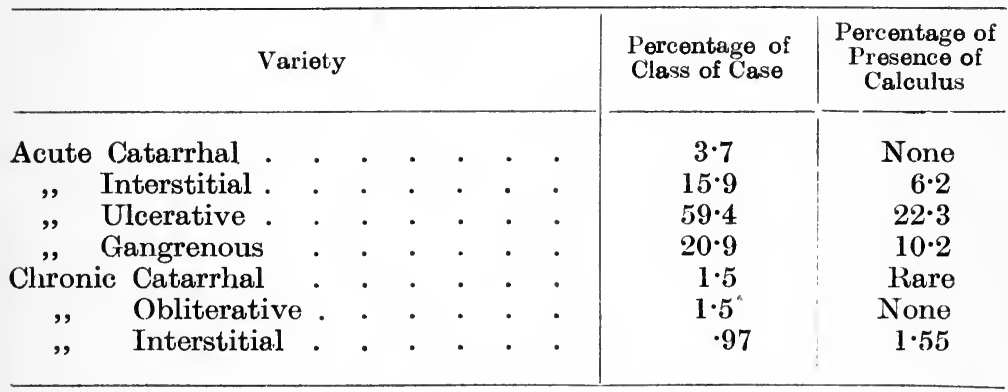

In the acute ulcerative and gangrenous cases the above figures represent the minimum, as numerous calculi are doubtless lost at the operation.

One of the most striking features about this table is the relatively high percentage of cases of interstitial appendicitis in which fæcal concretions have been found to be present. These appendices on account of the fibrosis of their walls cannot expel their contents by muscular contractions. 


\section{SURGERY OF THE APPENDIX}

Besides fæcal calculi and foreign bodies, worms are occasionally found in the appendix, such as oxyuris and ascaris. These are, however, distinctly uncommon, and may be reckoned as curiosities. Some authorities regard the appendix as the natural breeding ground of some of the parasites of infants.

THE VALUE OF LEUCOCYTOSIS IN APPENDICITIS.

By leucocytosis is meant an increase in the leucocytes in the blood, and it is estimated by a " blood count." The white corpuscles are augmented in number in many illnesses, but especially so when pus is present. Consequently the value of a blood count in appendicitis was expected to yield extremely useful indications of the clinical progress of a case. Unfortunately, the method has not turned out to be as valuable as was hoped. The following brief résumé embodies the main points at issue.

1. The leucocyte count is valuable in the prognosis; the greater the increase the greater the likelihood of obvious purulent complications.

2. An increasing, or a stationary and high leucocytosis demands operation.

3. A decreasing, or a stationary and low leucocytosis is safe. The import of the last two points must always be taken in conjunction with clinical symptoms.

4. There may be no leucocytosis with mild inflammations, chronic inflammations, extremely fulminating cases, extremely chronic and localised abscesses, etc.

5. It serves as an indication of the necessity of operation from the patient's point of view, and so is of value in deciding the question of immediate operation.

Though a leucocyte count may be of some value, especially to those of limited experience of these cases, the clinical 


\section{BACTERIOLOGY}

features form by far the most expressive and reliable indication for operative treatment. ${ }^{1}$

\section{THE BACTERIOLOGY}

A great deal of work has been done upon the bacteriology of this affection, though many problems remain to be worked out. Taking all the published series into account, the following brief summary may be made : Primarily the chief organism is the bacillus coli communis under one or other of its many forms. The bacteriology of the different clinical varieties exhibits certain differences that are well worthy of study in that they may afford some clue as to those conditions found.

\begin{tabular}{|c|c|c|}
\hline & $\begin{array}{c}\text { Acute } \\
\text { Appendicitis }\end{array}$ & $\begin{array}{c}\text { Chronic } \\
\text { Appendicitis }\end{array}$ \\
\hline 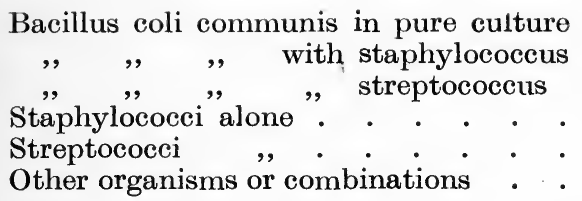 & $\begin{array}{l}70 \text { per cent. } \\
15 \text {," , } \\
7,, \quad, \\
4 \text { ", , } \\
\text { Very rare } \\
4,, \quad,\end{array}$ & $\begin{array}{l}90 \text { per cent. } \\
6,,,,, \\
\text { Very rare. } \\
1 \text { ", ," } \\
\text { Very rare. } \\
3,,,,\end{array}$ \\
\hline
\end{tabular}

It is thus seen that septic organisms are more common in acute than in chronic cases. These results are very deceptive, as a strong and hardy organism such as the colon bacillus is known to kill off its fellow bacteria. In this way its great frequency in pure culture is explained. Such might not be expected from the mixed community of organisms found in the disordered intestine. Streptococci are well known to be easily killed off, and are notoriously difficult to keep alive in the presence of other organisms. Hence their general and comparative absence. The staphylococci form a hardier group, and are not unfrequently found.

In the more difficult task of continuing the lines of Deaver's classification into the bacteriology of the different clinical

1 French, The Practitioner, 1904, p. 829-848. 


\section{SURGERY OF THE APPENDIX}

sub-classes, it is seen that septic organisms are found more commonly in cases of ulceration with perforation than without perforation, and more frequently still in cases of gangrenous appendicitis.

These remarks apply to both streptococci and staphylococci. Using this as a "straw to show the direction of the wind," it seems not improbable that the origin of the acute cases of appendicitis lies with the septic organisms, which injure the tissues and so give the colon bacilli their opportunities. The latter then very ungallantly over-ride and kill off their pioneer cocci.

This explains the frequency of pure cultures of the colon bacilli that are obtained, and also suggests that the presence of the colon bacillus in our intestines is to some extent a protective measure. And it is in our natural intestinal "backwater," the appendix, that the septic organisms, when they do obtain access to it, do the damage.

Other organisms are occasionally found in pure or mixed culture, such as the bacillus pyocyaneus, proteus vulgaris, bacillus prodigeosus, bacillus aerogenes capsulatus, etc.

Coverslip preparations made at the time of an operation for appendicitis with general peritonitis are of some slight value in helping to give a prognosis. The outlook is much worse according to the number of cocci or pyogenic organisms present. The clinical picture is obviously of far greater value.

It has also been shown ${ }^{1}$ that organisms multiply and increase in virulence, and their products in toxicity when enclosed in the appendix, but in some cystic appendices the organisms die out, the contents being found sterile.

1 Dieulafoy and Caussade, Bull. and Mém. de la Soc. de Méd. d'Hôp., Paris, 1897, 38, xiv. pp. 1356-8. 


\section{CHAP'TER III}

Non-Suppurative Appendicitis: Acute, Chronic; Symptoms, Diagnosis, Treatment-Appendicitis from Torsion-Appendicular Colic

\section{ACUTE APPENDICITIS WITHOUT APPARENT SUPPURATION}

AN attack of appendicitis is frequently ushered in by certain premonitory symptoms, amongst which the most frequent are a general feeling of being " not up to the mark," with headache, abdominal discomfort, irregular gastro-intestinal troubles, anorexia, dyspepsia, looseness of the bowels, or constipation. These symptoms are amongst those which chronic appendicitis may give rise to. And the more subacute the attack the more do the symptoms approximate to these. In point of fact, both pathologically and symptomatically the acute and chronic cases are examples of a similar septic process. In all diseases the acute cases are recognized first, and we are only just beginning to appreciate subacute and chronic appendicitis. Doubtless numbers of these are still put down as biliousness, etc. The associated condition of the bowels has always been of considerable interest, and has given rise to great difference of opinion. Constipation is associated with a drier than normal consistence of the fæces without abnormally great (or perhaps even less) bacterial growth. The colon is, therefore, merely irritated mechanically by the presence of scybala. Diarrhœa, on the other hand, is associated with moist fæces and luxuriant growth of organisms. 


\section{SURGERY OF THE APPENDIX}

Hence it is to be expected that inflammation of the appendix will be found more frequently in people who have attacks of diarrhœa rather than in those habitually constipated. This view serves to explain the greater frequency of the disease in men rather than in the other sex. The bacterial growth will set up a catarrhal colitis, which, as has been previously shown, will affect, most of all, the cæcum and appendix, in which an acute inflammation will be prone to occur; whilst in the colon only, chronic inflammatory changes are common. It has been recognized clinically that diarrhœa with appendicitis frequently signifies a more severe - i.e. more septic type of disease. And it may be pointed out that both are the consequence of the same septic process.

It is no easy task to give a clear and concise account of the clinical phenomena of a disease so various in its characters as appendicitis. Clinical observations and methods are at best coarse when compared with the fineness of the pathological. And as time has to elapse for the latter to act upon the general condition of the individual, and so become clinically recognisable, the former succeed the latter. Then, individuals react to different pathological changes in different manners. Consequently, it seems foolish to expect to be able clinically to recognize any but gross pathological differences. From these considerations we have been led to adopt the broad clinical classification of chronic appendicitis, acute appendicitis without apparent suppuration, acute appendicitis with local abscess, acute appendicitis with local spreading peritonitis, acute appendicitis with diffuse peritonitis. The types can be clinically recognised, and depend upon disease of wide differences of pathological degree. Beyond these simple limits pathological differences are not taken into account in the clinical classification.

The apparently non-suppurative class of appendicitis is by far the largest, and is extending more and more as chronic and subacute cases are becoming recognized. Examples of 


\section{ACUTE APPENDICITIS}

chronic appendicitis are discussed under their own heading, whilst only the subacute or acute cases are considered here. To begin with, it must be most strongly urged that generally it is impossible to form any sound judgment at the first, and that the most severe symptoms may crop up suddenly in apparently the mildest cases; it must also be remembered that there are some which resemble the changeable month of March, and "begin like a lion whilst they go out like a lamb." In only a small percentage of cases can the attack be definitely and reasonably attributed to an exciting cause, such as injury, exertion, etc. Such events usually merely draw the patient's attention to the local condition that has been already, perhaps only sub-consciously, in existence.

The first definite symptom to appear is undoubtedly pain in the abdomen, which varies considerably in character. In most cases it is described as being of a dull, cramping, or griping character, but may be so sharp and acute as to simulate renal or biliary colic. Generally, the pain is at first referred to the umbilical region, and in twelve or twenty-four hours' time becomes located in the right iliac fossa. The gall bladder and the epigastric regions are not uncommonly referred to as the sites of the pain. Before the patient localises the pain in the right iliac fossa, tenderness can almost always be obtained at McBurney's, or better Clado's point. Vomiting may occur once or twice a few hours after the onset, especially if the attack follows some dietetic indiscretion. In an uncomplicated case no further vomiting should take place. During a first attack this symptom is apt to be the most severe, and is most probably due to the involvement of peritoneum which has never been affected before. The bowels may act once, twice, or three times, and generally speaking diarrhoa indicates a more severe type of the disease. After this no further action occurs. The tongue, which is the great index to the condition of the alimentary tract, becomes rapidly covered with thick white 


\section{SURGERY OF THE APPENDIX}

fur, and remains moist. There is complete loss of appetite, and the breath is offensive.

The temperature is a character on which considerable stress has been laid. And though some general points may be inferred, yet in many instances it is most irregular and misleading. In the cases under consideration the temperature rises a little before pain is complained of, and may reach, within a few hours of the onset of symptoms, to a height of even $104^{\circ}$ or $105^{\circ}$. It is very unusual for rigors to take place, though occasionally they do, and then do not always seem of the serious import to be expected. The fever in these cases is usually at its highest at the end of the first or second day, and from that time on, it speedily declines. Unfortunately, an examination of the temperature charts reveals the fact that the fever in these cases is most irregular in type, and offers little criterion as to their future progress. In a few, defervescence takes place by crisis instead of the more typical lysis. These irregularities are easily understood if the fact is remembered that appendicitis is essentially a septic disease, and that these variations in the decline of temperature are very likely due to the perfect or imperfect discharge of an abscess into the cæcum, appendix, etc.

The pulse is raised correspondingly with the temperature to 90 or 100 , and even quicker, on the first day. The respiratory movements are almost entirely costal, and are increased in frequency, but not unduly so. The physical signs to be relied on are imperfect movement of the abdominal wall, at first general, later localised to the right iliac fossa, with tenderness and rigidity, soon followed by impairment of resonance in that region, and later by the presence of an inflammatory mass, at first soft and indefinite in outline, becoming harder and more distinct.

Together with these local signs are the general ones of raised temperature, pulse and respiration rates, the general appear- 


\section{DIAGNOSIS OF APPENDICITIS}

ance, the furred tongue, constipation, etc., which render the "diagnosis" in the male fairly clear. In the female, the right Fallopian tubes are the chief source of doubt. But never let there be omitted a rectal examination. When it is remembered that in far the largest number of cases the appendix lies internal to the cæcum, and in most of these is in more or less relation with the pelvis, the importance of this examination is clear. It is also emphasised by the not infrequent presence of the symptom of pain on micturition. A mass in the rectovesical pouch may be found, a tender mass on the right side, or perhaps only a tender point high up.

The progress of an uncomplicated case is usually that the pain and fever begin to abate by the third or fourth day, and both cease during the second week. The inflammatory mass may take several weeks to disappear; this long period is very likely due to the presence of some small quantity of pus amongst the adhæsions. The bowels, that were obstinately constipated at first, begin to act, the tongue cleans and the appetite returns.

Indicanuria is frequently to be found in these cases. The presence of indican in the urine is significant more of the presence of a chronic attack of appendicitis upon which the more acute attack has been grafted, than of the latter in a previously healthy appendix. Indicanuria seems merely indicative of fermentative processes in the intestinal tract, and may be associated with any lesion, gastric or otherwise, being in no way pathognomonic of appendicitis. It is found in ordinary constipation of the bowels.

Albuminuria and hæmaturia occasionally occur in the same attacks. Glycosuria is very rare, but subjects of chronic appendicitis will very likely have their saturation point to glucose lowered as in other septic diseases. (Mr. C. S. Wallace, Lancet, 1899, December 23.)

The Diagnosis is very far from easy in acute cases, and that 


\section{SURGERY OF THE APPENDIX}

of the most acute will be left to the section which will be devoted to the subject of acute diffuse general peritonitis. The more subacute, i.e. those which are apparently nonsuppurative, may be briefly considered in this place. The onset of acute appendicitis is exactly like that of any other acute abdominal disease, whether it begins in the stomach,

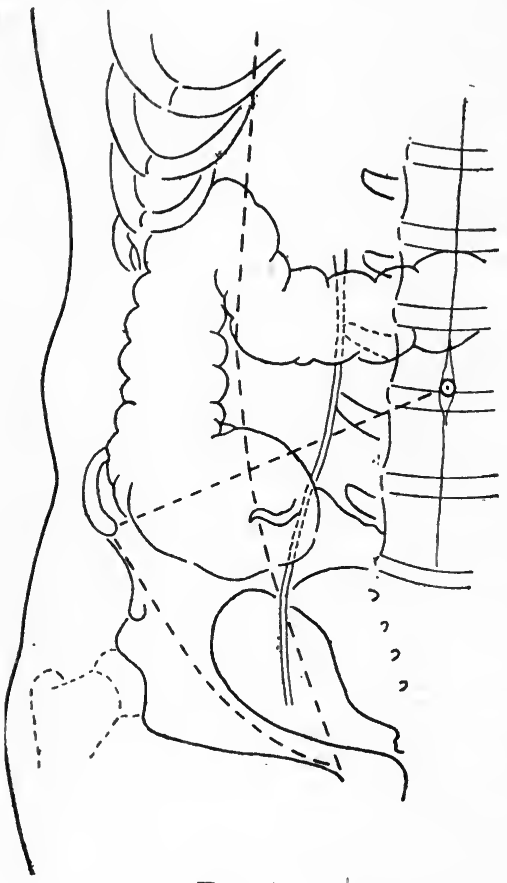

FIG. 4.

Representing the relationship of the appendix to the position of the ureter and abdominal wall. duodenum, or otherwise, and is characterised by a class of symptoms which Sir Frederick Treves has called peritonism, consisting in the main of shock, abdominal pain, and vomiting. At this stage of the diagnosis the origin of the disease is mere guesswork. Appendicitis is the most common of all severe abdominal diseases, and the majority of subjects are between 10 and 30 years of age. The pain and tenderness soon localise themselves in the right iliac fossa, and afterwards a resistance, at first indefinite, later becoming. a defined mass, can be made out. It is this class of case with which we are now concerned. As Prof. Howard Marsh in his Clinical Essays has said, "Probability is often the basis of a sound diagnosis," so may it be stated at once that the appendix is responsible for far and away the majority of such cases. Intestinal obstruction, especially in an imperfect degree, such as that which arises from adhæsions, bands 


\section{DIAGNOSIS OF APPENDICITIS}

chronic intussusception, etc., gives rise to similar symptoms, and may be distinguished by the persistence of the symptoms, the abdominal distension with visible peristalsis, the absence of fever, etc.

The diagnosis in subacute cases is frequently impossible. Examination of the pelvic viscera should always be made, as the right ovary and appendages often do enter into the diagnosis. Right salpingitis, right pyosalpinx, tubo-ovarian abscess, pelvic peritonitis, and perhaps parametritis, ovaritis, inflammation, prolapse or torsion of small ovarian cysts, tubal gestation, leaking or rupture and others have been diagnosed as appendicitis. (Fig. 4.) Diseases of the right kidney and ureter are of great importance, the most frequent of which to be mistaken is calculus in the right ureter. When in the ureter proper renal colic usually clearly indicates itself. But the mere movement of the stone whilst retained in the pelvis of the kidney gives rise to spasms of pain that are more easily mistaken. For instance, a boy was said to have had 20 attacks of appendicitis, and when operated on, the appendix was normal, but there was an oxalate of lime calculus the size of a marble in the pelvis of the right kidney. Subsequent inquiries elicited the fact that once the urine had been dark after an attack. Amongst other affections of the kidney may be mentioned movable kidney, early hydronephrosis especially if intermittent, small pyonephrosis, etc. An examination of the urine in these cases is of the greatest importance.

In a like manner stones in the gall bladder or ducts may give rise to attacks extremely like those of appendicitis, whilst on the other hand the symptoms of this disease may be referred to the region of the gall bladder. It may also be borne in mind that the fœtal position of the cæcum is under the liver, and that its subsequent descent may be delayed at any part of its course. Certain digestive troubles referable to the 


\section{SURGERY OF THE APPENDIX}

stomach may give rise to symptoms like those of appendicitis. In most of these cases the presence of a definite mass in the iliac fossa will practically settle the diagnosis. Besides the above, certain medical diseases such as typhoid fever and colic below the diaphragm, and pneumonia, pleurisy, etc., above the diaphragm, must be borne in mind. The extreme importance of the "respiratory" diseases is patent to all who have held hospital appointments in the last few years, and has been further emphasised by Mr. Barnard, in the Clinical Society's Transactions.

Treatment. It has been, and is, the custom to treat cases of appendicitis with milk, beef tea, etc. as food; rest in bed, fomentations to the abdomen, doses of opium. With regard to the opium, the least possible amount should be given, as it masks the symptoms, and gives a feeling of perhaps false security to the patient, the friends, and the medical man.

The custom with regard to the bowels has been generally to leave them alone for some days without any action. It is not an easy question, because in some cases the purgative has been blamed as doing harm, and in others, attacks have certainly been aborted by them. If the case is seen shortly after the onset, and the vomiting allows of it, a purgative is a good thing. It is almost commonplace to remark that after abdominal operations patients are much better and more comfortable after their bowels have been well open. And it is the custom in cases of peritonitis to do all that is possible to obtain free purgation. After the end of the first day the purgative must only be given with caution, and what is far more important, and what we should like to recommend as the most important line of treatment in all cases of appendicitis, is to watch carefully the signs. For at this time, within the first forty-eight hours, is what may be called the period of election for early operation. To arrive at this decision of operation or nonoperation is no easy matter, and if the practitioner can make 


\section{PROGNOSIS OF APPENDICITIS}

up his mind surely that no operation is needed, then a purgative may be given. But the considerations are such that an opinion may either be easily formed one way or the other, or else none can be. In the last case, that is when in doubt, it is safer from the patient's point of view to advise surgical intervention. To continue watching is sound, but it must be clearly formulated that early operation may be more valuable from the patient's point of view than continued observation, and the prognosis changed from good to bad by the loss of time.

If operation is called for after the third day, it cannot but be felt that the case would have been far better treated had it been so submitted earlier. Whilst we do not urge immediate, and therefore clinically indiscriminate operations, as the diagnosis will be in some instances most uncertain, we do urge that the decision for surgical procedure should be formed, whenever possible, within forty-eight hours of the onset, and that should be the aim and endeavour of the practitioner.

Of course this is difficult, and implies that the case will have been seen perhaps more than once within forty-eight hours of the onset, conditions which friends do not by any means always give the practitioner. Yet frequent examinations must be insisted on in these grave abdominal lesions, and no excuse for slackness permitted to any one. It would be most instructive if a series of cases could be published in which a decision was so made, and the further result of the cases recorded. In this way we should obtain a better and clearer knowledge of the probabilities of the disease than we have at present, where one set of men see mainly mild cases, and hospital and consulting surgeons chiefly observe severe ones.

Prognosis. The outlook for a case of appendicitis is a very difficult subject on which to express an opinion, and in the early stages any statement must be guarded. It is well known that the majority recover under medical 


\section{SURGERY OF THE APPENDIX}

treatment. And we also recognise that any of the complications, even of the most fatal kind, may arise in apparently mild cases. It is impossible to deny the possibility of almost any of these. An opinion can only be formed by watching the case and the progress of the signs and symptoms.

The first attack is the one in which the gravest complications are most prone to arise. The most important symptoms to watch are the pulse, the persistence of vomiting, and the progress of the abdominal signs. These suggestions are the result of considerable clinical observation, which has also bred a distrust of the temperature variations in many cases. Of course the normal pulse cannot always be known for each case, and in one man a pulse of 90 is equivalent to a pulse of 120 in another. Still, the character and rapidity of the pulse, with persistence of vomiting, and well marked abdominal signs, are the best indications that we have of the gravity of the case ; and in modern times may be regarded as indications for immediate operation. Far be it from us to recommend anything like indiscriminate operations, as then doubtless many cases of other abdominal disease or even pneumonia would be subjected to a needless appendicectomy. But in some eases it is better to advise early operation than to risk waiting. Naturally the earlier that this operation is undertaken the better the prognosis. If operation is not resorted to in the first three days, it should probably not be needed, unless an abscess occurs, or another attack supervenes, until the case has quieted down.

\section{SYMPTOMS THAT MAY RESULT FROM APPENDICITIS AND ESPECIALLY FROM THE CHRONIC FORM}

It has now become recognized that chronic inflammatory changes in the appendix may lead to far-reaching consequences which are only now attributed to their rightful source. To sum matters up shortly, the appendix, through chronic inflammation, becomes unable to completely empty itself on 
account of the resulting imperfect peristalsis. As a consequence it becomes a culture tube and incubator to all the organisms of the cæcum. This may cause clinically recognisable symptoms in two chief directions.

Firstly, from absorption of the toxins elaborated by bacterial action, primarily in the appendix, is associated a chronic toxæmia with lassitude, depression of spirits, heaviness of mind, general depression of health, of mind and body, with dyspepsia, constipation, colitis, etc. The liver, to which the toxins are primarily carried, becomes sluggish and torpid, and the conjunctivæ may assume a slightly icteric tint.

Secondly, the cæcum will become infected by the organisms and their toxins elaborated in the appendix. Owing to the "rest" of the products of digestion in the cæcum until their consistency becomes such that it can by its muscular contraction propel them onwards, the fermentative processes initiated in the appendix will proceed to further stages in the cæcum. As a result, a secondary subacute or chronic typhlitis is started which leads to interference with the muscular action of the cæcum and further retention of the contained fermenting fæcal mass. In this way a vicious circle has been started, and the processes and their results may extend along the colon from segment to segment. The clinical consequences of these changes will be diarrhœa and constipation in alternation. The more acute the process is the more will the former predominate, and vice versâ. Ultimately this results in colitis with the passage of mucus in the stools. A still further stage is ulcerative colitis. That this disease has been in many cases appendicular in origin has of recent years been proved. And it may account for the majority of such cases, though it cannot be urged as accounting for all. Even cases of apparently acute colitis have been reported as secondary to appendicitis.

This is, however, a very far-reaching subject, with a train of symptoms that may be brought about by other conditions 


\section{SURGERY OF THE APPENDIX}

than appendicitis, consequently it is impossible to be sure of, or to exclucie disease of that organ clinically. The modern tendency seems to be to operate more often than hitherto, and concurrently with this procedure comes the knowledge that. some cases are marvellously improved after operation, and on the other hand, some are not; and it is at present impossible to foretell which it will be. Unfortunately, the question of improvement depends upon multiple factors, primary and secondary local conditions and primary and secondary mental states. Hence the index for our judgment is unreliable, and it seems that a distinction must be drawn as to what is scientifically or empirically suited best for the material to be worked with. Of the cases with the general symptoms described which have definite tenderness on deep palpition of the right iliac fossa, and perhaps also on rectal or vaginal examination repeatedly made, these seem to yield the best results from operation. And it is well to remember the connexion between the appendicular and ovarian lymphatics through the appendiculo-ovarian ligament, and examine the right ovary for fibrous changes.

The treatment for this condition is not always easy or satisfactory. As the appendix may be in many cases the primary focus of the disease, and in every case it will be secondarily affected, so by its chronic inflammation and consequent organism harbouring capacity it will aid materially in keeping up or increasing the condition, that organ should be removed. In early cases great benefit may accrue from this. But unfortunately, cases, apparently more common in women, come up for treatment after they have been suffering several years; and in whom the involvement of the large bowel, the typhlitis and colitis, is such that the involvement of the appendix, though primary, is now a mere incident in the disease. Still the appendix is primarily or secondarily a fons et origo mali, and in all operative treatment for these complex 
conditions should be removed. The mere removal of that organ followed by prolonged medical treatment in the shape of diet, attention to the bowels, etc., is frequently sufficient to yield great improvement in health. But where the affection of the large bowel is not amenable to medication, etc. further operative treatment is needful, such as establishing a temporary artificial ileostomy through which the large bowel may be flushed out and irrigated, or if the appendix has not been removed, and admits of it, the stump may be sutured to the skin and used as the means of washing out the colon, as advocated by Dawbarn.

\section{APPENDICITIS BY TORSION}

When the appendix has its distal end free and movable, it may be so carried by the movements of the coils of small gut that it becomes bent on itself so sharply that the distal part sloughs. Such an event is very uncommon, and clinically presents all the characters of an attack of acute or even fulminating appendicitis. The appendix may be rotated upon its long axis as well as upon itself. The process may be briefly described as the first stage of a knot into which the organ may be tying itself. The most recently described case is that by Lilienthal, ${ }^{1}$ which was characterised by two severe hæmorrhages from the bowel, which he correlated with the torsion of the appendix, although the connexion is not quite clear. It was supposed that the hæmorrhage took place into the proximal part of that urgan, and was so discharged per rectum. The bleeding certainly ceased after the removal of the appendix. These hæmorrhages were not noticed in the cases recorded by Routier $^{2}$ and Broca. ${ }^{3}$

\section{APPENDICULAR COLIC}

It was for a long time considered that the appendix could

1 Lilienthal, New York Medical Journal, Feb. 7, 1903 (Case II).

2 Routier, Bull. et Mém. de Soc. de Chirurgie, Paris, 1901, xxvii. 631-2.

3 Broca, Gaz. Hebdom. de Méd., Paris, 1896, xliii. 1026. 


\section{SURGERY OF THE APPENDIX}

not give rise to attacks of colic unless there was, or had been definite change in its structure or position, almost invariably the results of inflammation. That such is not always the case is proved by the following case under the care of Dr. Minchin, on which one of us operated. The patient, a boy aged fifteen, had suffered from attacks of pain in the abdomen at intervals since the age of four. Some of these attacks had been sufficiently severe to necessitate his keeping to his bed, but not the attendance of a medical man. They generally developed after some unusual exertion. The last attack continued three days, and he complained of aching pain of a severe paroxysmal character. There was no vomiting; the temperature was normal, and nothing abnormal could be detected in the right iliac fossa. The diagnosis of an abnormally large appendix was made, and at the operation it was removed and found to be eight inches in length, with well developed muscular coats, but without trace of disease. After removal it disposed itself in a curious snake-like spiral round the mesentery as a central support. The patient has had no further attacks of abdominal pain. Dr. MacDougal has described a case in which a most painful and alarming attack followed the impaction of a grain of barley in the appendix. In one case sent to the hospital by Mr. Boxall, of Rudgwick, the patient, a young girl, stated that she had suffered from at least a hundred attacks of severe pain during the previous three years. From her account some of these had been of a more serious nature than mere colic. Yet the appendix only presented evidence of chronic catarrhal inflammation after removal.

When the appendix is diseased such attacks may be common, and will be associated mainly with chronic inflammatory changes. As our knowledge of the pathological conditions of the appendix extends so must we realise that appendicular colic is not an uncommon occurrence. It is scarcely ever 


\section{APPENDICULAR COLIC}

primary and usually secondary to some functional stenosis of the lumen due to kinking by contractions of the mesentery, and so forth. Its association with the presence of concretions is not so common as is thought, these foreign bodies being so generally associated with a fibrotic condition of the muscular coats. Strictures are almost always close to the tip of the appendix, and therefore unable to give rise to colic, but those near the cæcal end may give rise to pain. An oblique insertion of the appendix into the cæcum will render the mucous membrane at that spot valvular (the valve of Gerlach), which may give rise to painful contractions in the attempts to pass the contents into the cæcum. The existence of tortuosities of the appendix are rarely congenital, and almost always secondary to some slight inflammatory attack which leaves little or no obvious changes behind it. And if they are associated with a long appendix, such as in the case related, colicky pains are extremely likely to arise. The advent of an acute attack is likely to so injure the coats of the appendix that colic can no longer occur.

The cases under discussion are most frequent in persons who have no leisure to attend to more than the most serious forms of illness, and unless they are brought to account by a severe and perhaps even fatal attack, they escape medical notice. 


\section{CHAPTER IV}

The Various Methods of Operation for the Removal of the Appendix Vermiformis in the Quiet Period

IT is, of course, not possible to mention all the modifications that have been suggested in the operation of removal of the appendix, or as some term it, appendicectomy. We have endeavoured to summarise the more important of them.

This subject may be considered under two headings-

(a) Entrance of the abdominal cavity.

(b) Amputation of the appendix.

The incisions made through the abdominal wall may be divided into three groups: (1) simple incision through all the structures in a direct line without regard to the planes of muscle; (2) incision of superficial structures, separation of the muscular fibres of external and internal oblique muscles, with incision of deeper structures ; (3) incision of the superficial structures, incision of sheath, and temporary displacement of rectus muscle.

1. Simple incision. Sir F. Treves was the principal advocate of this method, which is described as follows in his Students' Handbook of Surgical Operations, page 385 -

"The incision should be made obliquely from above downwards and inwards over the cæcal region, its lower extremity ending just external to the epigastric artery. It is as a rule most conveniently placed about two fingers' breadth internal to the anterior superior spine of the ilium. The incision should not be always made directly over the appendix, or over the dullest regions. If it be so placed a number of adhæsions will 


\section{METHODS OF REMOVAL}

probably be encountered, and the demonstration of the peritoneal cavity might be difficult. The cæcum or appendix might be actually adherent to the anterior abdominal wall. The incising of the peritoneum should therefore be conducted with the very greatest care. It is well that the parietal cut should open the abdomen at a point just beyond the diseased area where no adhæsions exist."

Another method is to incise the abdominal wall through the rectus muscle. This presents certain disadvantages compared with others, which have prevented its employment on anything like a large scale.

2. Incision of superficial structures, separation of muscular fibres. Of the methods under this heading the operation of McBurney ${ }^{1}$ is probably the most widely known and practised. It is a good method, and under the name of the gridiron operation has met with large success-

"Beginning at a point one inch above the line drawn from the anterior superior iliac spinous process to the umbilicus, passing obliquely downwards, an incision is made which crosses that line at a point $1 \frac{1}{2}$ inches internal to the spinous process, and corresponding as accurately as possible in direction to the fibres of the external oblique muscle and aponeurosis. Separation of the fibres of the external oblique is now made in a line corresponding to the skin incision, and no tendinous fibres are cut. Excepting in fat subjects, the incision just described need not be of greater length than two or three inches. The edges of the wound in the external oblique aponeurosis are to be pulled apart with retractors, when the surface of the internal oblique muscle will be seen, the fibres of this latter crossing the superficial incision nearly at right angles. At a point opposite the anterior superior spine of the ilium, the muscular sheath of the internal oblique is to be divided with scissors in

1 International Text-book of Surgery, vol. ii. p. 415. 


\section{SURGERY OF THE APPENDIX}

a line parallel with the muscular fibres. With blunt-pointed scissors or the handle of a scalpel the fibres of the internal oblique and transversalis muscle can now be separated, without division, in a line parallel with their course-that is, nearly at right angles to the incision in the external oblique aponeurosis. When the separation is complete, the fascia transversalis will be seen lying at the bottom of the wound. Blunt retractors should be introduced through the line of separation, and the edges pulled apart until a considerable area-an inch or more in diameter-of fascis transversalis can be distinctly seen. If the opening through these two muscles appears too small, it can readily be enlarged by introducing the two forefingers, and by direct traction still further separating the muscles to almost any extent desired. The outer surface of the fascia transversalis, with the peritoneum closely adherent to it, now comes readily into view. With fine-toothed forceps these membranes may be lifted, either separately or together, and an opening made with the scissors. Through this opening one blade of the scissors is introduced, and the opening enlarged, first towards the middle line of the body, and then outward toward the anterior spine of the ilium. Excepting from the small vessels of the skin and subcutaneous tissue, no bleeding will occur, and no muscle nerves of any size will have been injured. The appendix having been removed and its stump disposed of, the wound in the peritoneum and fascia transversalis is to be closely sutured with fine catgut. Although the fibres of the internal oblique and transversalis muscle fall

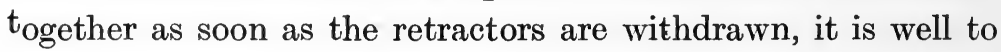
make their apposition complete throughout the whole depth of the wound with a few fine catgut stitches. The incision in the external oblique is also to be sutured from end to end. Complete restoration of the elements of the abdominal wall is thus made; and if aseptic healing takes place, absolutely no impairment of the strength of the abdominal wall will result. 


\section{THE " GRIDIRON" OPERATION}

The patient may leave his bed on the thirteenth or fourteenth day."

Wyeth (Medical Record, 1902, pp. 892-3), in considering the various methods for the removal of the appendix, says that he uses only two, McBurney's and the clean cut through the abdominal wall. He is of opinion that after the former operation the patient should spend two to three weeks in bed, and after the latter five to six weeks in bed, before being allower to get up.

Elliott (Boston Medical and Surgical Journal, 1896, p. 433) has this modification. He begins with a horizontal incision half an inch inside the anterior superior spine, and carries it to the linea semilunaris. He cuts in the same direction across the external oblique, and makes a great point of not stripping this up. He splits the internal oblique and transversalis. If this incision is not large enough he extends it either way along the linea semilunaris.

Fischer (Annals of Surgery, 1897, p. 624) recommends an incision one inch above and parallel to the iliac crest, beginning at the outer edge of the external oblique and going forward to a point corresponding to the anterior superior spine. He splits the external and internal oblique muscles.

Fowler (Medical News, New York, vol. lxxvi. p. 34) writes of " a new improved method of entering the abdominal cavity in the ileo-cæcal region." He advises a curved incision, which commences just above the anterior superior spine, extends horizontally to the edge of the rectus, then parallel to this downwards. The external oblique is divided and the anterior sheath of the rectus. This muscle-the rectus-is retracted, then the internal oblique and transversalis separated along the course of their fibres.

Weir ${ }^{1}$ (International Medical Congress, Sect. de Chirurgie Générale, Paris, 1901 ; Compte Rendus, pp. 801-4) describes

1 Also Med. News, New York, lxxvi, 1900, p. 241. 


\section{SURGERY OF THE APPENDIX}

his modification of McBurney's operation, which, he thinks, fails for want of room. If necessary, he strips up the external oblique as far as the rectus sheath, which he opens, retracts the muscle, and then opens the posterior sheath transversely, sewing them up afterwards. This is practically the method of Dr. Morris (Lectures on Appendicitis, 1897).

W. Meyer (Journal American Medical Assoriation, vol. xxxiv., 1900 , p. 385) suggests a hockeystick-shaped incision, the full account of which is not quite clear; but he commences half an inch above and midway between the anterior superior spine and McBurney's point and carries the incision nearly down to the position of the femoral artery, separating the fibres of the internal oblique, etc. By this means he considers better access is given to the pelvic organs on the right side, and diseased structures can be removed without further incision.

Beck (Sammlung Klin. Woch., p. 98) favours an oblique incision which runs in a straight line and course from the end of the eleventh rib towards the os pubis.

Sonnenburg operates as for ligature of the external iliac artery.

3. Temporary displacement of the rectus abdominis. The operation which we advocate is one founded on the anatomical structure of the abdominal wall in front. In this operation the rectus muscle is temporarily displaced, and after the appendix vermiformis has been removed the muscle mechanically returns to its normal position as regards the other structures. It may be taken for practical purposes that the inner threefifths of the distance between the anterior superior iliac spine and the umbilicus is covered by the rectus muscle, that muscle extending much further outwards than is generally recognised. The distance from the anterior superior iliac spine to the outer margin of the rectus sheath is really only about two inches, and if there is any important adhesion of the appendix, or it is much displaced, or there are any complications, such as 


\section{TEMPORARY DISPLACEMENT OF MUSCLE}

adherent omentum or disease of ovary or tube, they cannot be dealt with easily, if at all, through an incision which, without invading the sheath of the rectus, is of such limited extent as that in McBurney's operation.

The average distance from the anterior superior spine of the ilium to the centre of the umbilicus, as measured in patients of both sexes from the age of twenty to thirty, is $5 \frac{1}{2}$ inches. These measurements were taken by Messrs. Downes, Spurrier, and Hudson, from a series of 100 cases in St. Thomas' Hospital.

This operation is performed as follows, after the usual preparation of the patient: An incision is made through the skin, and subcutaneous tissues down to the sheath of the rectus; this section is made obliquely downwards midway between the anterior superior iliac spine and the umbilicus, is about four inches in length, vary-

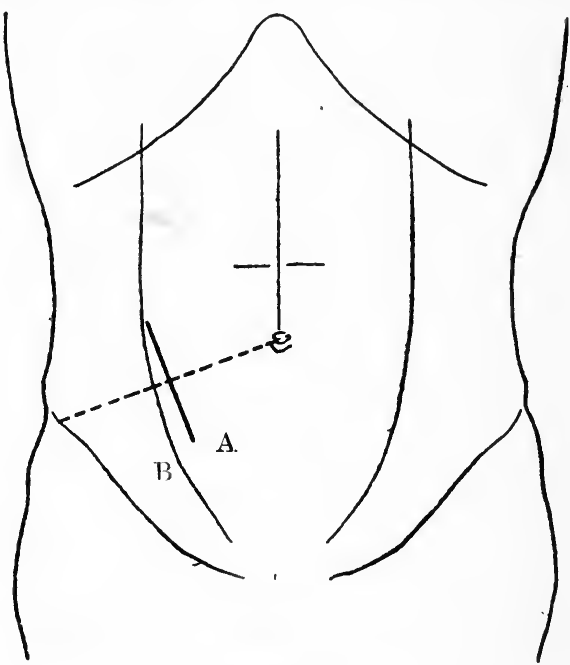

FiG. 5. ing with the deposit of fat in the abdominal wall of the individual, To illustrate the position of the superficial incision with regard to the rectus muscle in this operation. A, line of incision. B, linea semilunaris.

and is placed so that it is equally extended above and below this line. The outer margin of the rectus sheath can now be easily defined, and during inspiration the direction of the action of the external oblique aponeurosis seen. The inner margin of the wound is retracted about half an inch, and the anterior sheath of the rectus opened close to the retracted inner margin for the full extent of 


\section{SURGERY OF THE APPENDIX}

the incision. The rectus muscle is now drawn inwards, being separated from the outer part of the sheath with a few touches of the knife. Occasionally one of the lineæ transversæ requires to be separated from the sheath. Running across the posterior layer of the sheath can usually be seen the dorsal nerves, with accompanying vessels. The deep epigastric artery and vein run upwards under the retracted muscle to the inner side, and it is well to define these vessels, otherwise the vein may be punctured in the later suturing of the sheath. Another anatomical point of importance is the level of the transversalis fascia as regards the wound, for it strengthens the posterior layer of the sheath and makes the hold of the sutures much firmer, if the opening into the peritoneal cavity can be confined to the part covered by it. In most instances the incision can be made through the posterior sheath between the nerves and through the fascia. We have not, however, seen any ill-effects from division of these nerves, although it has been suggested that partial atrophy or paralysis of the rectus muscle might ensue. If the case is uncomplicated by any adhæsions, and the appendix is lying in a normal position, the operation can be completed through an inch-long incision of the posterior sheath; but if there are complications, and it is considered necessary to do so, the wound, both superficial and deep, can be extended to any required distance. After the appendix has been removed by the method which is considered best, and the peritoneum cleansed, the posterior layer of the sheath and peritoneum can be sutured in the usual manner-a continuous suture of silk ( $\cdot 00)$ or sterilised catgut in the case of females; whilst in the male, from the more fixed character of the peritoneum, it is frequently necessary to use silk (or sterilized catgut) of a larger size and interrupted sutures. During this suturing, the omentum must be prevented from becoming entangled in the sutures, which it has a great tendency to do. The uninjured rectus is now permitted to 
fall back into its usual bed; the anterior layer of the sheath is closed by three or six interrrupted ( $\cdot 00)$ silk sutures, and the skin and superficial wound by a continuous horsehair stitch. Union takes place throughout by first intention, and the horsehair stitch is removed on the eighth day.

This method of operation is most valuable in cases of removal of the appendix " $\grave{a}$ froid," i.e., in the quiet period, and from a large experience of it the authors can affirm that it answers admirably. It gives ready access to the caput cæci, and from it the appendix is easily reached and removed. It has been -wrongly, we think-called the indirect method by Dr. McDougall, for it is not uncommon to find the appendix show directly the incision through the peritoneum has been made, and when traction is made on it and upon the cæcum the base of the former is directly under the middle of the wound. It was first performed by one of us on January 1, 1895, for the removal of the appendix in a patient (of Dr. W. R. Macaulay), in whose case it was essential that no weakness of the abdominal wall should remain.

So far, in our hands we have had no loss of life in the absence of previous suppuration about the appendix, and have seen a hernia follow this operation in only one case-that of the wife of a medical man, in whom there was extensive local suppuration after the removal of a very adherent appendix which necessitated a trying operation. The cause of the suppuration was not evident, but was probably to be ascribed to a continuous catgut suture which was employed, the sterilisation of which was possibly not satisfactory.

The patients are allowed to get up on the tenth day after operation, and usually leave on the fourteenth. They require no belt afterwards, or other means of support for the abdominal wall. The scar is slight, and in a few months can hardly be seen.

The method of operation by displacement of rectus muscle is referred to by Mr. C. B. Lockwood, in his monograph on Surgical 


\section{SURGERY OF THE APPENDIX}

Diseases of the Appendix, as the method recommended by Mr. A. A. Bowlby. There are two records of interest in this regard-one in the Clinical Journal of November 28, 1894, in which operation at the linea semilunaris is advocated, which is not the same by any means; and another in the St. Bartholomew's Hospital Journal, April, 1896. Here he advocates this method, having carried it out successfully on December 30 , 1895 , almost a year after the date referred to above. The result of this operation had previously been demonstrated by one of us at a meeting of the Clinical Society in the spring of 1895. We understand that he now makes the incision as near the middle of the anterior layer of the sheath as possible. This, we think, is a mistake, because the muscle is displaced with greater difficulty, and it requires a more extensive disturbance to make sufficient room. The incision should be about an inch to the inner side of the linea semilunaris.

Jalaguier, of Paris, in that year (1895) did a similar operation, not knowing at the time that one of us had previously done it. This method is now known in France as la méthode de Jalaguier.

The operation which Schüller performed in 1889 was apparently founded on the same idea of the value of displacement of the rectus in abdominal operations. In his operation the incision was, however, made parallel to the outer border of the rectus, and ran one finger's breadth to the inner side of the centre of a line spanning the anterior superior spine and the symphysis pubis. He does not appear to have troubled about the anterior layer of the sheath.

Kammerer (Annals of Surgery, 1897, p. 224) has suggested an operation which is practically the same as the one performed by one of us in 1895 .

Lennander (Centralblatt fur klin. Chirurgie, 1898, p. 90) is later in the field, and others have followed him.

Quite recently it has been put forward as a new operation, 
but from careful investigation we think it had its origin in St. Thomas' Hospital.

When the peritoneum has been opened a finger should be passed into the abdomen to find out the position and condition of the appendix and the surrounding structures, also the presence or absence of adhæsions and undue swelling, such as might indicate the presence of a small collection of pus. Should the appendix be free in the abdomen and easily brought to the surface, a strip of gauze should be placed in the wound and over the exposed visceral peritoneum, only enough being brought outside to permit of safe manipulation.

Usually the surgeon's first consideration is the division of the meso-appendix. This structure varies very much in its thickness and length, and also in the amount of fat enclosed in its layers. In general, if the inflammatory changes have been slight, it is easy to pass a double ligature through the angle of the mesentery and tie above and below, afterwards dividing between with scissors. If this does not appear wise on account of the thickness or unusual length of the appendix, it should be ligatured off in segments, two or three usually sufficing. Sometimes it may be better to apply forceps to the bleeding points whilst the surgeon snips through the mesentery from the proximal attachment, the various points being afterwards ligatured with 00 sterilized silk or catgut. It is very usual to find an unsecured artery running close to the muscular layer of the appendix at the reflection of the peritoneum, which requires ligature. We have described the method which we consider the best at page 83 .

Not infrequently on first inspection the appendix appears to be without a mesentery, the peritoneum crossing from side to side like a transparent cloak, through which a limited part of the appendix can be seen, the remainder being buried in adhæsions, the result of a former attack of inflammation 


\section{SURGERY OF THE APPENDIX}

\section{B. Amputation of the Appendix}

The methods of amputating the appendix are numerous, and must receive consideration, though it is hardly possible to do more than mention some of them. Still, in these days of activity, unless the more important are mentioned, we may any day find a description of a supposed new method which has been tried and abandoned years ago. In the early attempts, after due precautions had been taken to prevent infection of the peritoneum by the contents of the appendix, a simple ligature was applied to its base, and, after it had been cut off, some strong antiseptic or Paquelin's cautery was applied to the mucous membrane. This led to a number of cases of perforation during the after-treatment, and a high mortality.

A second method is inversion of the ligatured stump into the caput coli. Then Van Hook has practised amputating between a temporary ligature and distal forceps with cauterization of the stump with carbolic acid, and the application of a row of Lembert sutures tied after the removal of the temporary ligature.

Dawbarn recommended the use of a purse-string suture, applied a quarter of an inch from the appendix base in the serous and muscular coats. The opening into the cæcum from the stump was then stretched with forceps and the stump invaginated; the suture is tied as the forceps is withdrawn. In some cases of thickening of the cæcum this method would be both difficult and risky, the suture tearing out.

Another plan is to cut through the peritoneal and muscular coats, excise the mucous membrane, and suture the opposing muscular and serous coats.

McBurney's method of cutting away the appendix very close to the colon, then pushing in the margins of the opening with a probe, over which a double row of catgut or silk sutures is passed, is well known. The coat-sleeve method of Mr. A. E. Barker is one which has received much support in this country. In this a circular incision is made through the peritoneal coat 
about a quarter-inch from the cæcal junction, and a cuff of peritoneum turned down; a ligature is then placed round the other coats near to the opening into the cæcum, and the amputation completed. Afterwards, pure carbolic acid is applied to the stump, any excess washed away, and the peritoneum and muscular coat ligatured over the inner part.

Deaver cuts off flush, and sews up with two layers of sutures the edges of the wound and the peritoneum.

We quite agree with Dr. Haggard (Transactions American Medical Society, 1898), when he says the proverbial untrustworthiness of the thermocautery has ever been the bete noire of the practical surgeon, and join him in condemnation of its use in this and other abdominal work.

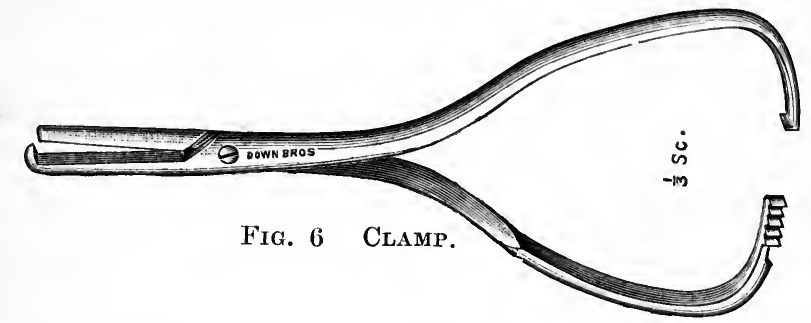

Formerly the method we used, as a rule, was the coat-sleeve method of Barker, sometimes scraping away redundant mucous membrane with the sharp edge of a knife from the ligatured stump before invaginating it, and then suturing. We now, and for two years have employed a plan which necessitates the use of a special clamp, as follows.

The appendix having been freed and its mesentery secured with one or two pairs of artery forceps, the clamp is applied to the base of that organ at its junction with the cæcum. The mesenteriolum is then ligatured, after which the large clamp is removed, and it will be found that it is possible to see through the appendix where it has been compressed. A fine silk ligature is tied as close to the cæcum as possible, and the appendix cut away with scissors. A piece of tissue scarcely larger than that 


\section{SURGERY OF THE APPENDIX}

ordinarily ligatured with an artery is left to be sewn into the cæcum. The rationale is much like that of ligaturing an artery with rupture of its coats. The clamp divides the mucous membrane and muscular coats, leaving the peritoneal surfaces in apposition for about one-third of an inch. The orifice into the cæcum is plugged by the ruptured coats. It may be claimed for this method that-

1. It is the quickest, neatest, and most aseptic method of removing the appendix.

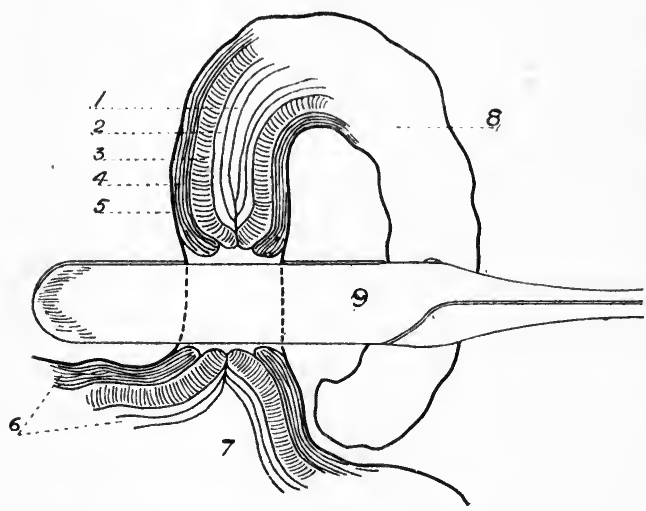

Fig. 7. The Extent of the Application of the Clamp on the Appendix.

1, Mucous coat. 2, Submucous. 3, Muscular. 4, Subperitoneal. 5, Peritoneal. 6, The divided and retracted lavers to the proximal side of the clamp. 7, The cæcum. 8, The Appendix. 9, The Clamp.

2. It leaves behind the finest of ligatures and the minimum of strangulated tissue to be buried in the cæcum, and this should, if possible, always be done.

3. It is especially applicable in cases of inflamed appendix where the organ is swollen and the walls of the cæcum are too sodden to hold stitches well.

4. It is of alnost universal applicability which other methods are not, and dispenses with the "drop of pure carbolic."

Sometimes, when the appendix is brittle in consequence of change throughout, and the peritoneum is no longer elastic, the clamp may cut across it almost like a knife ; this rarely happens, and then the stump can be treated by one of the methods mentioned above. 


\section{METHOD RECOMMENDED}

If the wall of the cæcum surrounding the part to be invaginated is very stiff, great care must be exercised in tying any sutures, for they readily pull out, and may give if any strain is put on them. Sometimes it is not possible to invaginate without excision of a portion of the wall. 


\section{CHAPTER V}

The Complications of Appendicitis

LOCALISED PERITONITIS WITH THE FORMATION OF PUS. CIRCUMSCRIBED SUPPURATION. APPENDIX ABSCESS. PERITYPHLITIC ABSCESS

IT is not possible to say in what percentage of cases of appendicitis suppuration will ensue, for there are many which do not come under the observation of the profession. Many cases are not recognized and still more never apply at a hospital for treatment, and are not, therefore, available for statistics.

More cases of localized suppuration are sent for admission now than a few years ago, but the proportion which this class bears to other varieties of the disease is not apparently higher. The following are the figures for St. Thomas', of cases treated on the surgical side of the hospital-

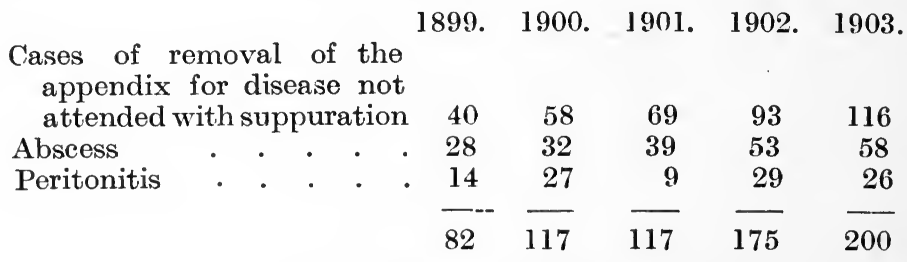

On one day in the year (1903) Dr. Hector Mackenzie had no less than four cases admitted for treatment, in each of which there was, or had recently been, suppuration.

The conditions of the appendix which give rise to localized suppuration in the peritoneum near are found to be "ulceration due to the presence of a concretion, often culminating in necrosis of the appendix wall; and (2) an acute necrosis of 


\section{DIAGNOSIS OF ABSCESS}

the wall consequent upon a chronic catarrh. Here commonly a simple chronic catarrh without necrosis or a cystic appendix may prove sufficient to excite the local formation of pus (Hawkins, p. 84). It is extremely probable that the explanation given by Dr. C. R. Box ${ }^{1}$ for the formation of gastric ulcers applies equally to some of the ulcerations which are met with in the appendix - that they are due to microbic infection of lymphoid follicles.

The apparent cause of an attack followed by localized suppuration is the same as that which occasionally appears to be responsible for an ordinary attack-unusual exertion, the jolting of a patient during a motor ride, a sudden strain when lifting, an indigestible meal, possibly a violent aperient, exposure to cold and wet, etc.

Although a case in which a very acute appendicitis has developed is more likely to end in suppuration, still it is not possible to say, even in a case of mild onset, that an abscess will not form. As we have already said, there are no certain means of telling.

The diagnosis of abscess will depend on signs with most of which the surgeon is familiar when dealing with suppuration in other parts of the body, modified by position and surroundings. The way in which the illness commences-a sudden attack of pain in the abdomen, which becomes localized to the right side in the iliac fossa, accompanied by fever and probably by vomiting, and followed by the formation of a swelling, persistence or an increase of symptoms instead of a decline after thirty-six or forty-eight hours, rigidity of the overlying muscles. The swelling is very tender, and to it the patient refers the pain. Very rarely an abscess forms quickly and in two or three days fluctuation may be demonstrated, but frequently in the early stages the only local sign of value as indicating the presence of pus is excessive tenderness, and the

1 Brit. Med. Journ., Feb. 8, 1902. 


\section{SURGERY OF THE APPENDIX}

surgeon would be wrong to wait for the evidence of fluctuation before deciding that an abscess is present. It must be remembered that rupture of an abscess in consequence of too much handling is not unknown.

The use of an exploring needle has been advocated, but British surgeons agree with Sir Frederick Treves in his condemnation of its use ; it is dangerous and unreliable in this disease as in other abdominal conditions when used as an aid to diagnosis. It is much more satisfactory to administer an anæsthetic and examine the patient when he is under its influence. A little gas and oxygen will usually suffice. In all cases the rectum should be examined with the finger.

If the iliac swelling persists and there is hectic fever, although fluctuation cannot be made out, pus is assuredly present.

Again, when a case of iliac phlegmon has developed an unusual rise of temperature, in an otherwise normal course, suppuration should be suspected,or a fresh attack of appendix inflammation.

If when the patient is seen for the first time there is œdema of the abdominal wall, with redness of the skin, and even bulging, there can be no doubt of the diagnosis, but such an extreme state of things is rarely met with at the present time. Appendix abscess nowadays rarely perforates the abdominal wall ; it is, however, occasionally seen, the pus forming a water cushion under the skin, and when displaced by pressure of the finger, a rounded smooth opening leading to the appendix region through the muscles can be felt.

Sometimes there is a history of rigors, of more or less severity, and in the case of longer illnesses, a history of night sweats.

When the suppuration is of limited extent, especially if pelvic in position, or forms part of a large exudation, it may be overlooked, and the first suspicion of its presence may be a discharge of pus from the rectum, and a sudden fall in the temperature to normal. 


\section{DIAGNOSIS OF ABSCESS}

Flexion of the thigh on the abdomen is a sign which occasionally is found as a consequence of contraction of the psoas muscle. This may result from an irritation of the muscle, or from interstitial inflammation of its substance. Dr. Hawkins gives an illustration of the microscopical appearance of a portion of muscle so affected. Lameness, with flexion of the limb, may persist for some time after recovery, even in cases where there has been a simple inflammation only. In children left-sided symptoms are not unknown.

Occasionally the abscess is concealed, that is, there are none of the signs present on which we rely to show that there is a collection of pus. After an attack of inflammation in the region of the appendix the temperature may become normal, and the general and local conditions greatly improve. But there remains some swelling which is firm and not particularly tender. This swelling may contain pus and before operation it may be quite impossible to distinguish it from some of those cases in which there has been an intense local inflammation with thickening of the surrounding structures. It is common to find a small amount of dry caseating pus when operating for the removal of the appendix after some of the more severe attacks. The larger kinds of concealed abscess are found behind the cæcum, in the subcæcal fossa, running up along its outer side, and in the pelvis. Examination per rectum has sometimes shown the presence of an abscess in the pelvis due to disease of the appendix which has caused symptoms mistaken for those of typhoid.

An accumulation of pus in the appendix region may sometimes pass into the general peritoneal cavity in consequence of the giving way of some adhesions which were not firm enough to withstand a sudden strain, possibly as the result of vomiting. A sudden collapse, followed by the signs of suppurative peritonitis, with a rapidly fatal ending, would usually be the result. In the majority of cases when the abscess bursts spontaneously, 


\section{SURGERY OF THE APPENDIX}

it discharges into the larger bowel, the rectum is the most common part selected, but the cæcum and ascending colon are sometimes chosen as well as the smaller gut. In the female it may point in the vagina, and either be opened by the surgeon or burst unexpectedly.

Bladder symptoms, frequent micturition and pain, are not infrequent as a result of extension to the wall of the bladder of inflammation, having its origin in the appendix, especially when that structure is pelvic in its position. Sometimes when the other symptoms have subsided, these remain for a time in consequence of adhæsions. The discharge of the contents of an abscess into the bladder may cause much pain and disturbance, passing into a definite cystitis.

The pus may perforate the abdominal wall, present in the lumbar region, make its way into the thigh, or find its way along the spermatic cord into the scrotum, and require opening there. A man who had recently had this experience was admitted under the care of Dr. H. Mackenzie in 1903, and his appendix was removed after a further attack of inflammation.

The result when an abscess opens into the bowel is not unfavourable-most cases recover. But if the escape of pus is not free, and this discharge is accompanied by fever, abdominal distension, and hæmorrhage from the bowel, the resemblance to typhoid fever will be very close, and there is great risk that the patient will die from exhaustion.

When an exudation remains stationary (as far as can be judged) for an unusual period of time, or shows evidence of increase in size, also, if instead of becoming less severe, the pain becomes worse, then pus should be suspected. The influence of the nervous element must not be overlooked at times, and undue importance attached to the presence of pain and tenderness, for inflammatory swellings of the omentum or intestinal coils near the origin of the disease are often slow in resolving.

These abscesses are not often allowed to reach the surface, 


\section{SYMPTOMS OF ABSCESS}

but occasionally they do so, and present all the classical signs of pointing, there being usually considerable odema of the abdominal wall and great tenderness. There may be tympanitic resonance on percussion, pointing to the presence of free gas in the swelling; this and the dullness due to fluid will be found to vary with the position of the patient, and there may even be a splashing or gurgling sound.

The position of the appendix in relation to the other structures near, the direction in which it is lying when it becomes diseased, will influence to a great extent the site which will be occupied by an abscess. This varies considerably from a point below the liver to one in the pelvis. When it is lying over the brim of the pelvis, an abscess will form according to the part of the appendix which gives rise to the suppuration, a localized lesion near the base may cause an abscess in the iliac fossa and one nearer the tip, either at the pelvic brim or in the pelvis. The pus finding its way by gravitation and yielding of imperfect adhesions to the bottom of the Pouch of Douglas, until a very large abscess may be formed with few indications of its size. The irritation of the surrounding tissues gives rise to the formation of the pus, and it does not accumulate like water falling from a leaky tap into a bucket, as suggested by one writer. Appendix abscesses are frequently of irregular shape, with pouchings in various directions.

When the appendix has been lying behind the cæcum and ascending colon it is still an intraperitoneal organ, but as a result of acute inflammation and sloughing, pus may form in the cellular tissue behind the peritoneum and a large abscess be formed. It is not possible to say when this abscess is out. side the peritoneum and when it is within, lying to the outer side of the colon, and if the patient is very stout it may be altogether overlooked. The origin of the abscess can usually be correctly named when the pus is being evacuated, either from its offensive odour or from the presence of a concretion. 


\section{SURGERY OF THE APPENDIX}

Several questions need answering when we consider the treatment of this kind of abscess after a diagnosis has been made.

In the first place it is necessary to decide whether it is best to open the abscess at once, although it is small, or whether we should leave it until it has got nearer the surface and of a considerable size.

In the earlier days of the surgical treatment of diseases of the appendix, when the opening of the peritoneum was seldom done purposely, being regarded as a very dangerous procedure, there was an agreement to wait until it was probable that adhesion to the abdominal wall had taken place and the peritoneum been shut off. 'There can be little doubt, from what one remembers of the results of abdominal surgery of the time, that this was the safer practice; but with greater experience and improved methods we no longer fear infection of the peritoneum being able to prevent it.

By operating on the abscess early we may anticipate its rupture within the general peritoneal cavity, remove the appendix, and probably avoid a fatal peritonitis. We prevent the formation of a large abscess, the escape of the contents of which into one of the hollow viscera may give rise to prolonged and exhausting illness.

The effect of suppuration in a wound of the abdominal wall on the subsequent formation of ventral hernia is well known and it is advisable to make the incision in such a manner that the muscular fibres are separated and not cut across. In a few instances it will be well to treat the abscess as an abscess elsewhere would be treated, and cut down on the most prominent part, where in fact it is pointing. Round this spot, which may be very minute and varies much in extent, the viscera will be adherent to the parietal peritoneum, and directly the latter is incised pus will escape. As the layers of the abdominal wall are encountered they will present evidences of in- 


\section{TREATMENT OF ABSCESS}

flammatory exudation, and it may be difficult to recognize the deeper layers from the amount of matting and œedematous infiltration affecting them. Care must be exercised at this stage, for if the knife is used, under the impression that the peritoneum has not been reached, the adherent bowel may be opened. The better plan is to use the finger in the deeper parts of the wound, the end of a pair of closed Spencer-Wells artery forceps, or the point of a director. When the abscess, where it is pointing, has been opened, we sometimes wash out the cavity gently with sterilized saline solution, and search for concretions, or a sloughed appendix. A perforated rubber tube of adequate size is then introduced.

It may be asserted that these patients will make a satisfactory recovery if the tube is kept in long enough to thoroughly drain the abscess. It is inadvisable to look for the appendix if not found in the pus, for although not doing so will necessitate a second operation, a search is attended with unnecessary risk to the patient, and may be unsuccessful in the end, especially if placed towards the pelvis. In the case of very large abscesses, or when anterior drainage is not sufficient, a posterior incision should be made in the lumbar region, and a second drainage tube introduced.

When examination shows a tender codematous bulging of the wall of the rectum towards the front, the incision should be made here. The patient having been placed in the lithotomy position, and a speculum introduced into the rectum, a longitudinal incision is made over the most prominent part dividing the mucous membrane, the deeper parts being opened up by the passing of a director along which a closed pair of Spencer-Wells forceps is passed, opened and withdrawn. This will suffice to make an opening large enough for the escape of pus and the, necessary drainage. After the evacuation of the abscess and cleansing of the parts as much as possible, a strip of antiseptic gauze is introduced and left in for forty-eight 


\section{SURGERY OF THE APPENDIX}

hours. Before operation the relations between the bladder and the abscess must be ascertained and carefully noted.

A "similar method of operation may be required should the abscess be found to point in the vagina.

From the very offensive character of the pus in appendix abscesses, it is advisable to wear rubber gloves when operating, otherwise great difficulty will be experienced in getting rid of the offensive smell, which is annoying and persistent. (Peroxide of hydrogen is useful to remove this.)

Most frequently the abscess points above the outer part of Poupart's ligament, and the incision required runs parallel with that structure.

In dealing with an abscess rising out of the pelvis the surgeon must remember that there is a danger that the bladder may be placed in the middle line in front, covering or partly covering the swelling caused by the matted intestine, and omentum, having been carried up with it as it became larger. Operation in the middle line over the most prominent part of the abscess would probably lead to damage of the bladder, possibly to its perforation. In such cases it will be best to make the incision in the right linea semilunaris low down, avoiding or tying the deep epigrastic vessels. A catheter should be passed in all such cases, not only to empty the bladder, but to define its position.

The possibility of a rupture into the rectum, intestine or peritoneum during the manipulations preceding the operation, or as a result of examination per rectum, must be borne in mind. Should it have occurred, a sudden change in the tension of the abscess sac will be noticed, and renewed examination of the rectum by the finger of the assistant may show the presence of pus there. In one case of the kind in our experience, the patient made a good recovery, the abscess bursting into the rectum during the examination on the operation table after the anæsthetic had been given. No operation was then 
performed, but the appendix was removed at a later date with most satisfactory result. He is now able to do a hard day's work in the saddle, and has had no further trouble.

If when the parietal layer of the peritoneum has been incised, examination shows that the abscess is not adherent to it, and all that is found is a lump covered in by coils of adherent omentum and intestine, the incision should at once be made of adequate size, and the area which it is proposed to attack carefully solated by strips of sterilized gauze or abdominal sponges. The finger should then be gently insinuated between the nearest coils of bowel, or if the omentum is covering the abscess, from the direction of the anterior superior spine or from the iliac fossa towards the centre of the swelling. By this means the edge of the omentum is raised and none of the covering of the abscess disturbed. Much care is required in this separation if the adhesions are of a firm character. The pus will present the usual characteristics, and the cavity should be treated by washing out and cleansing with small sponges; for whilst it is usually possible to reach all parts in a small cavity, it is not so in a larger one where there are irregular pocketings, and ill-regulated pressure might easily separate adhesions, and start a fatal spreading inflammation of the peritoneum. We cannot speak too strongly of the necessity for careful isolation by packing before search is made for the pus. After cleansing the cavity and the parts within, the barrier of gauze is removed and the parts again washed. In some earlier cases the parietal peritoneum was then sutured round the opening to the underlying bowel, or omentum, but this is not necessary if gauze is carefully packed round the tube, as it passes from the abscess, to shut off the general peritoneal cavity. Search should be made for concretions; if left in the wound they may cause a persisting sinus, and also for a sloughed appendix, although this is not often found in a localized collection of pus. 
In these cases it is permissible to make a definite attempt to remove the appendix, for the area of operation is under control and there is less risk of doing harm. Much will depend on the size of the abscess and the general condition of the patient.

The gauze strips should be kept in situ for forty-eight hours and then removed, the abscess cavity being treated by daily syringing with 1 in 2,000 perchloride of mercury solution at each dressing, or with some other antiseptic solution in which the surgeon has confidence. Wood-wool pads will be useful to receive the discharge. It is a good plan to use a drainage tube with the gauze, for if there is bleeding, the plugs become saturated and will fail to act as drains, and prevent rather than expedite the escape of fluids, as the coagulation of the blood in the meshes of the gauze prevents any capillary action.

In January, 1902, one of us published a paper on the advisability of removing the appendix vermiformis after suppuration caused by appendicitis.

For some time the question had occupied the authors' attention as to whether the appendix vermiformis should be removed after suppuration had been caused by its disease--that is, in cases where, for some reason or another, it was not removed at the time when the operation for the evacuation of the pus was performed. The opinions held which govern the treatment of the appendix in these cases differ greatly. The first is, that if a patient has an abscess as the result of appendicitis there is such a profound destruction of parts that the surgeon need trouble no more about the case-when once the abscess is closed the patient is cured, for the appendix is obliterated. This view is rather generally held.

Not long ago, a well-known surgeon, Sir W. Mitchell Banks, is reported to have said, ${ }^{1}$ when taking part in a discussion on appendicitis: "As soon as the suppurative stage has been diagnosed the pus should be evacuated when the appendix was 1 The Lancet, February 23, 1901, p. 554. 
practically destroyed, and such patients had no return of appendical trouble." Dr. McBurney (International Text-book of Surgery, vol. ii. p. 422) says : "The appendix is frequently completely destroyed by the suppurating process. Prolonged search for it in such cases would, of course, be purely harmful. If it has not been destroyed, it is frequently obliterated by the subsequent process of repair. Not infrequently have patients been sacrificed to excessive zeal in handling the cavity of these abscesses, and this is especially true of children. The only very important point is to give free exit to the pus; and after this has been done, the less the operation is prolonged the better."

The second view held by some is exactly the opposite of the first, and may be summarized as follows : that in every case of perityphlitic abscess the appendix should be removed at the time of the operation. This is strenuously advocated by Dr. O'Connor, of Buenos Ayres.

Mr. Rutherford Morison ${ }^{1}$ has devised a method of operating which he has successfully practised on several occasions, by means of which he considers this should be done, and of which the mortality is said to be small. It gives free access to the iliac fossa, and the area of operation is well under observation, so that search is made for the origin of the disease under the best circumstances. The operation involves an extensive incision of the abdominal wall, and even to surgeons of large hospital experience appears somewhat formidable, whilst one cannot regard it as free from great risk of subsequent hernia. There is however one thing admitted which tells against it more seriously, and it is this, that in pelvic cases it may not be possible to find, much more remove, the appendix. In other words, the operation may be unsuccessful and a serious risk uselessly undergone, for it is not possible to select the successful cases beforehand.

Again, other surgeons hold the opinion that whilst it is advis-

1 Ibid., p. 533. 


\section{SURGERY OF THE APPENDIX}

able to remove the appendix when the abscess is opened, there are cases in which such a procedure would involve unnecessary risk to the patient. There are many conditions, any one of which in a particular case may appear so important as to induce the operator to postpone the removal of the appendix. For instance, the patient may be too ill to permit such a prolongation of the operation as might be required; the appendix may be so placed that it cannot be found; it may be considered that removal would involve too rough manipulation of bowel already softened by inflammation, the adhæsions being old and very firm ; or some areas of healthy peritoneum might be unnecessarily and without knowledge opened to infection from the abscess cavity.

There appears to be a want of unanimity regarding the necessity for getting rid of the appendix if it has not been excised at the first operation, but some cases described below strongly emphasize, we think, the advisability of removing it. Moreover we have heard of some very painful cases in which the appendix was not removed and a later attack of peritonitis has proved fatal. Again we have not met with a pathologist who could say that he had not been able to find the appendix after a careful search, nor have we ever seen an instance of its obliteration as a result of suppuration outside it.

For a long time it was the authors' impression that the advocates of the first view were correct, but circumstances have convinced us to the contrary, and the appendix should be removed as soon as possible after the healing of the abscess. Faith in the value of the general statement that removal is unnecessary was shaken, in the first place, by the formation of a second abscess (some months after the first had been treated by one of us) in the case of a boy, aged 16. The first abscess was a large subacute one with much surrounding exudation, and the pus was already making its way through the 


\section{REMOVAL AFTER ABSCESS}

abdominal wall when the patient was first seen and the abscess incised. Relying on the prognosis which had been taught as the correct one, his parents had been given the assurance of his freedom from further trouble of the kind. Some time after this, a young female patient was being operated on in the hospital. As the expression of her face indicated acute abdominal mischief, further inquiry was made. She had had a perityphlitic abscess opened six months before in a large hospital. The appendix was now in a condition of acute inflammation with sloughing, but contained no concretion. There was diffused septic peritonitis secondary to this state of the appendix, and she died some hours after the operation. A valuable life would have been saved had the appendix been removed in the quiet period. It is not right to wait for symptoms.

Even if the appendix does not induce a second attack of suppuration it may cause a renewal of attacks which will make the patient's life a burden, and prevent his following his employment.

It may be considered that if a fæcal concretion is found in the abscess cavity the removal of the appendix need not be so strongly urged. It must, however, be remembered that there may be more than one concretion in the appendix, and all of them may not have escaped out of the ulcerated opening. Of the average number of concretions in an appendix we have, so far as we know, very little information : there may be two or three. The difficulty once met with in getting a patient well was explained ultimately by the presence of three concretions : two were removed from the abscess with the pus, but a third remained behind in the appendix, and caused recurring abscesses.

A labourer, aged 40, was transferred from a medical ward to the surgical side of St. Thomas' Hospital, on July 10, 1899. It was noted that he had been taken ill on June 28, with abdo- 


\section{SURGERY OF THE APPENDIX}

minal pain and vomiting. There was also a history of attacks of pain in the side of the abdomen, which he called "stitch," for a period of four years. On admission a swelling was present in the right iliac fossa, evidently an abscess. At the operation an incision was made through the right rectus sheath and the muscle displaced inwards. The abscess extended up behind the cæcum and the ascending colon, being shut off by adhesions from the general peritoneal cavity. Two fæcal concretions were removed from the abscess cavity, which was irrigated and drained. The appendix was not found. He had still a sinus when sent to a convalescent home on July $26 . \mathrm{He}$ was readmitted in September, 1899, and a stitch was removed; the sinus was also scraped. The track closed, but he was readmitted in November with another abscess, which was opened. He was again admitted on February 2, 1900, for a sinus which had followed a recent abscess in the old situation. On February 22 the appendix was excised; it was adherent to the pelvic wall and was separated with some difficulty, its walls were much thickened and in some parts cicatrized, and it contained a fæcal concretion. The wound was closed without drainage and healed without suppuration.

In another case a concretion left in the appendix was found to have been the cause of repeated attacks of suppuration, two of which were not clearly localized, and both were very dangerous. The recovery from the first attack without sinus, with an appendix left in situ, containing a fæcal concretion, over which ulceration and perforation had probably taken place and then healed, is especially worthy of notice. It should certainly have been removed at the first operation, but the surgeon had probably good reasons for not prolonging it, and his decision was apparently justified by the recovery of the patient.

A girl, aged 14, was admitted into the Beatrice Ward of St. Thomas' Hospital, on December 21, 1900. It was stated that she had had an operation for peritonitis in May of that 


\section{REMOVAL AFTER ABSCESS}

year, that on recovery from this she was sent down to a Brighton convalescent home, where in July she had an abscess of the right side of the abdomen. She was taken ill again four days before admission to St. Thomas' Hospital, with abdominal pains and vomiting. The pain had not been very severe nor was the vomiting distressing. The abdomen was not rigid or distended and moved well on respiration. She said that she had pain which was referred to the right side of the lower abdomen underneath the right rectus; this pain was increased on pressure and there was some rigidity of muscle induced by firmer pressure. The pulse was good, the temperature was $100.2^{\circ} \mathrm{F}$., the tongue was clean, and the bowels had acted after a dose of castor oil. There were two scars in the lower abdomen-one made at the first operation in the middle line below the umbilicus, the other in the right linea semilunaris. With such a history, and in spite of the slightness of her symptoms, immediate operation was indicated. About two hours after admission the appendix was removed through an incision to the inner side of the outer of the two scars, the rectus muscle being displaced inwards. On opening the peritoneum a quantity of thin pus of dirty yellow colour and fæcal odour escaped. The appendix, which was bound down with old adhesions, was somewhat difficult to find and to separate; it contained a fæcal concretion and over it there was a ragged ulcer communicating freely with the peritoneal cavity. There were no recent adhæsions, the neighbourhood of the appendix was washed with saline sterilized water and the abdomen closed. The old scar in the linea semilunaris gave way a week later and discharged a small quantity of offensive pus for about a week, otherwise convalescence was not retarded.

If the concretion which first caused the outbreak of suppuration remains in the appendix it may be the cause of a fæcal sinus, as it was in the following case. It is rather interesting, however, to note that the ulceration had healed, and the sinus 
did not lead directly into the appendix at the time of operation. Yet who can doubt that other attacks of inflammation and possibly suppuration would have followed the presence of the concretion, had it caused fresh ulceration, when there was in addition such a definite stricture of the appendix near the opening into the cæcum.

A soldier, aged twenty-two, was admitted into St. Thomas' Hospital, suffering from intestinal fistula, on July 31, 1899. He had had attacks of appendicitis in March and August, and later, in November of 1898, had been landed at Port Said from a troopship and treated in the Lady Strangford Hospital for abscess. The appendix was not found when the abscess was opened. The following day the dressings were saturated with fæces and pus. A fortnight later he also passed pus by the rectum. Convalescence was retarded, but with the exception of the fistula he was in fair health when he left the care of Dr. E. Cuffey (who kindly supplied his notes). The discharge from the fistula, usually small in amount, was profuse and yellow after exercise or excess in diet. A hard, collar-like lump could be felt under the scar in the right iliac fossa, and to this the probe directly passed. The operation was not performed until other methods had been tried without benefit. The appendix contained a fæcal concretion, of about the size of a cherry-stone, situated about a quarter of an inch from the base, whilst to its proximal side there was a complete stricture. The wound was sutured in layers so as to remedy the weakness of the scar which showed itself as a result of the prolonged suppuration, and he made a rapid recovery, the abdominal wall being without weakness some months later.

Should the concretion escape from the appendix but not come away with the pus it may cause the formation of a sinus which will not heal until it is removed. Some years ago one of us operated on a pelvic abscess in a young 
girl and could not find the appendix or any cause for the abscess. A sinus formed in the lower part of the wound, and persisted for some months. Thinking this was possibly due to a concretion which had been caught in the drainage tract, the sinus was carefully sounded. A concretion was struck, the urethral forceps introduced, and with some difficulty it was extracted in fragments. After this the sinus rapidly closed.

When a fistula which is not fæcal persists, it is most commonly the result of irritation from a ligature or suture, and removal of this by means of a curette with subsequent packing of the wound will suffice to cure it. Here there is not necessarily disease of the appendix. In the following case the sinus persisted until the appendix had been removed. The sharp bend in it caused by the angle at which it was adherent to the abdominal wall produced the same effect as that of a stricture of its lumen.

A carman, aged twenty-two, was transferred from the care of Dr. Hector Mackenzie to the surgical side in St. Thomas' Hospital, on March 1, 1901, for a large abscess in the right side of the abdomen, in which situation the patient had complained of pain for a period of six weeks. The pain had not been sufficiently severe to prevent his doing his work, although it had been fairly continuous until a fortnight before, when it became severe, and he had rigors and vomiting. The swelling extended from the middle of the iliac fossa to the lower ribs and backwards into the loin. It was very tender and fluctuating. Incision was made in the lumbar region and a large amount of very offensive pus was evacuated. The cavity was washed out with saline fluid and a drainage-tube was inserted. $\mathrm{He}$ left the hospital well on March 20, excepting for a sinus in the lumbar region, from which there was little discharge. $\mathrm{He}$ returned to have the sinus scraped in April, but little benefit resulted from this, and he was readmitted for removal of the appendix on May 15. The oblique incision with displacement 


\section{SURGERY OF THE APPENDIX}

of the rectus was used. The appendix was adherent to the abdominal wall about four inches above the anterior superior spine. The omentum was adherent to the iliac fossa. A bullet probe was passed to the point at which there was adhesion of the appendix, but did not penetrate the entire thickness of the abdominal wall. The adhæsions were dense and required a long incision to deal with them. The appendix was thinned where adherent to the wall, it was the subject of chronic catarrh, bulbous in shape, and curled up on itself. In order to remove it, it was divided near the cæcum, turned up, and dissected out. He had a good deal of vomiting after the operation and some stitches in the anterior part of the wound gave way, but he left on June 20, having quite recovered.

It will be observed that the opening at the tip of the appendix had closed before it was removed. This was after the prolonged rest in bed and careful dieting. Few doubted who saw the appendix that indiscretion in diet or over-exertion would have brought on another attack of inflammation.

We think there is only one exception to the rule that the appendix should be excised in all cases after the patient has recovered from the suppuration, if it was not found possible or advisable to remove it when the abscess was opened. This exception is when the appendix has sloughed off and is lying free in the pus or comes away during the healing of the abscess -not a very common occurrence when the suppuration is localized. There is sometimes more than one concretion, and there is no way of proving the exact condition of things without removal of the appendix and examination of its interior. These concretions are not faceted like gall-stones, so that it is not possible to say from the examination of one whether others are left or not.

It may be held, we think, that the evacuation of pus from an appendix abscess may be safely done through an ordinary 


\section{REMOVAL AFTER ABSCESS}

incision; that it is not necessary to complicate the operation by any procedure which increases the risk, and that the removal of the appendix at a second operation, when the parts are quiet, will give a better chance to the patient in many, if not most, of the cases than if a removal is unwisely attempted or persisted in at the first operation.

The adhæsions which form after the inflammation of the surface of the apposed coils of intestine are not necessarily numerous, strong or vascular ; indeed, it is a matter for surprise to find in many so little change from the normal condition of the parts. 


\section{SURGERY OF THE APPENDIX}

\section{CHAPTER VI,}

Appendicitis with Spreading Peritonitis-Value of Individual Symptoms

\section{PERITONITIS}

ALL forms of appendicitis are accompanied by inflammation of the peritoneum; in the majority of cases this is accompanied by an exudation which forms a distinct swelling about the appendix and is still called by some a perityphlitis. A second form is that already described, in which the exudation becomes purulent though remaining localized. The third is the condition which is meant when "peritonitis" is spoken of at the bedside. In this an inflammation spreads to a variable extent from the appendix to other parts of the peritoneum, and is a far more serious complication.

Acute peritonitis may be a direct consequence of a gross infective lesion of the appendix producing such an intense infection of the surrounding parts that any protective exudation is melted away and the general peritoneal sac involved. The condition may be due to gangrene or perforation associated with a concretion, or to a minute focus of suppurative disease of the wall of the appendix; and in some cases of early operation the appendix will present a brilliant array of colours, from bright red to green, purple and yellow, the result of an acute general involvement of that structure. Sometimes it will be tense, full of pus, and on the point of bursting, whilst at others it will be found floating as a mere slough separated from its former attachments. 



\section{SURGERY OF THE APPENDIX}

$A$, mid-point of longitudinal section to ex-A pose concretion shown in Fig. 9.

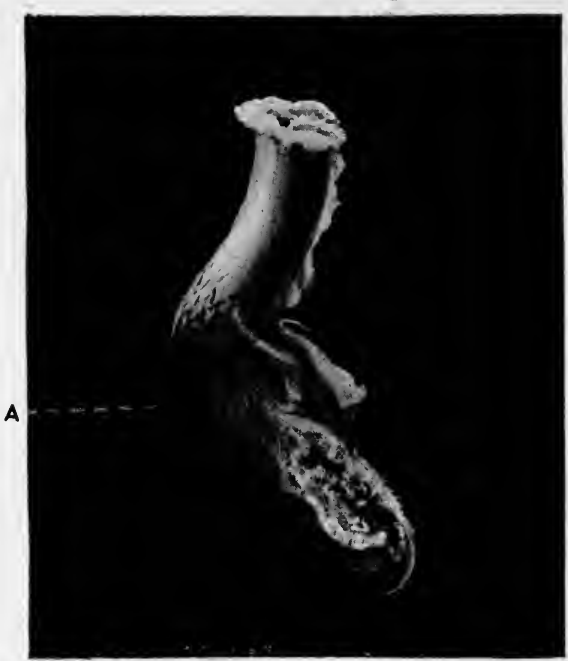

F1G. 8.-Appendix (half size) showing localized gangrene with perforation secondary to a fœcal concretion (see $p$. 109).

Removed from a child of $3 \frac{1}{2}$ years, in whom general peritonitis had followed localized suppuration.

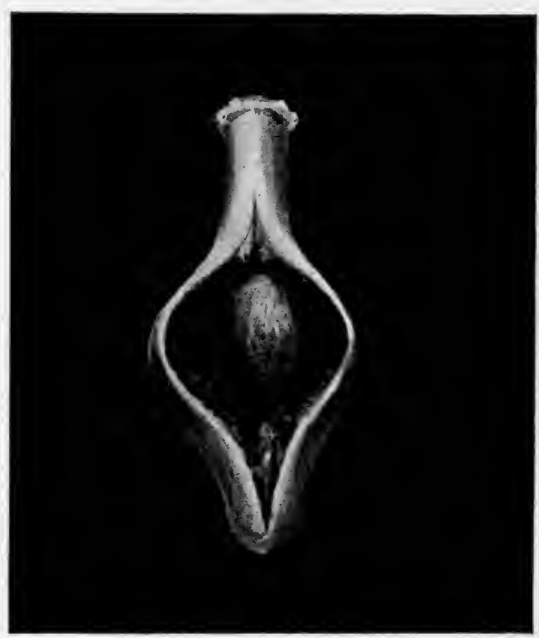

Fig. 9.- Position and relative size of concretion as shown by ircision at $A$, Fig. 8 . 


\section{PERITONITIS}

Fatal peritonitis may also occur in a secondary manner. A patient suffering from a localized suppuration in the appendix region, which is apparently progressing satisfactorily, and presents nothing unusual, although lying in bed surrounded by every care, may suddenly, without evident reason, complain of increased pain in the abdomen, and pass into a serious state of collapse from which the most prompt operation may be useless to afford more than temporary relief. Occasionally this giving way into the general peritoneal cavity may be of the nature of a gush of pus from an abscess, which floods the peritoneum to a variable distance. Here there may be a sudden cry of pain followed by collapse. Usually the change is more of the nature of a slow escape of germ-laden purulent fluid, and the symptoms are those of a general peritonitis gradually developing.

In our experience these cases of secondary spreading peritonitis are mostly those in which the appendix has become gangrenous, and it is not surprising, for the exuded fluids are then very septic, so much so that it says much for the protective power of the individual when pus resulting from a gangrenous appendix becomes definitely limited in its spread.

Most instances of peritonitis due to disease of the appendix are the result of a first attack of inflammation of that organ. The changes in the surrounding peritoneum probably account for the diminished fatality in cases where there have been previous attacks. There is also another reason, which is that the parents of a child in a first attack do not know what the symptoms indicate, and probably put off sending for their medical man until it is too late. We have known a case where the history given by the parents was that of bilious attacks, for which they did not call in assistance, and when a fatal attack of peritonitis came on with (in their judgment) the same symptoms, no medical aid was sought until forty-eight 


\section{SURGERY OF THE APPENDIX}

invaluable hours had elapsed after the onset of the illness. The so-called bilious attacks-(abdominal pain with vomiting) - had been mild recurring attacks of appendicitis.

The symptoms of a well-developed attack of peritonitis can scarcely be mistaken, but they are so liable to variation that two cases are seldom seen alike-and it must be remembered that peritonitis following a perforation of the appendix, does not differ in its essential characteristics from that due to a perforation in other parts of the intestinal tract, or from the giving way of an abscess of the pelvis or of other parts into the peritoneum.

The typical case of peritonitis presents local and general symptoms, the latter chiefly resulting from the absorption of bacterial products from the cavity of the peritoneum. In other words, there is a local inflammation affecting a large area of the body, one supplied to a marvellous extent with nerve ganglia and lymphatics. It is not a cause of surprise, therefore, that the progress of a septic inflammation in this part of the body is extremely rapid, nor is it strange that the resulting vital depression is profound.

The patient, in a case of appendicular peritonitis, will be found lying on the back with thighs flexed on the abdomen, and possibly the arms thrown up above the head; the expression is anxious, the eyes sunken and surrounded by dark rings. There is pain in the abdomen, and the abdominal wall is found to be quite fixed, not moving with respiration; there is considerable tenderness all over it, a touch on the skin causes an expression of pain, whilst the most gentle percussion is hardly tolerated. The muscles are hard and rigid so that nothing can be felt beneath them.

There is complaint also of frequent vomiting and inability to retain nourishment; the vomited material is watery, and of a greenish colour. The tongue is dry and furred, the breath foul, and the bowels are usually confined. There is restless- 


\section{PERITONITIS}

ness with inability to sleep, rapid, feeble pulse, and a rise in the temperature $\left(101^{\circ}-103^{\circ}\right)$.

In some cases the tenderness is greatest on pressure over the iliac fossa, and there is increased resistance in that part, or perhaps a defined swelling. Distension of the abdomen comes on, and increases in amount, whilst the abdominal wall is less resistant, and percussion may show the existence of free fluid. Restlessness, with a more anxious expression, thin, drawn, flushed face, sunken eyes, an effortless vomiting of large quantities of fluid is seen, the heart flags, and in time fails so that the pulse becomes small and uncountable, the extremities cold and clammy; free perspiration breaks out on the general surface of the body, the temperature becomes subnormal, and the patient, who has remained quite conscious throughout, suddenly expires. A cessation of pain and a drop in temperature, with some general improvement in the patient's condition not unfrequently takes place before the end, and gives rise to false hopes in those watching the case ; this improvement is, however, but temporary, and death is sometimes preceded by a period of excitement.

The surgeon had, for many years, chances of idly watching the progress of these cases of peritonitis, only too frequently called "idiopathic," for their nature was not understood by the physician, or the possibilities of relief by operation by the surgeon.

Amongst the numerous examples of the disease which present themselves in different stages at the present time, there are some which show that the main type still exists, and is extremely fatal. The late Dr. S. Fenwick stated that during a period of forty years only nineteen cases of "diseased appendix" were recorded in the post mortem registers of the London Hospital. ${ }^{1}$ Recent statistics show how much more material is now available for clinical study (see p. 86).

1 Lancet, 1884, vol. ii. 


\section{SURGERY OF THE APPENDIX}

The type suggested as common would probably cause no trouble in its recognition, and diffuse peritonitis would be immediately diagnosed and treated whenever found if all cases were alike. But it is not so, especially in the early stages; therefore it is necessary to consider carefully some of the more important modifications, and the value of certain individual symptoms. No disease can occasionally present a more insidious onset and progress, and it may not be until a sudden change for the worse reveals the extent of the mischief, and awakens (frequently too late) the unsuspecting friends and practitioner to a sense of the impending fate, that the real state of affairs is appreciated. We know of no disease which it is sometimes so difficult to appreciate, or in which more depends on a correct diagnosis, than peritonitis secondary to disease of the vermiform appendix.

In most cases the illness begins with an attack of acute pain in the abdomen, generally referred to the region of the umbilicus, and it may be that only after a few hours have passed will the pain be found to be referred to the iliac fossa, and a suspicion aroused that the appendix has anything to do with it. The fact that a sudden pain of considerable severity may result from so many conditions must always render the surgeon cautious in giving an opinion when this is the only complaint, even if accompanied by a state of collapse. In the early hours of the attack this pain is probably accompanied by a general tenderness which does not assist the examiner; but as the pain passes to the iliac region so the tenderness will be most marked there. It is seldom that an operation is required on account of pain alone, but a case under the care of Dr. Hector Mackenzie showed an exception to this rule. The case was that of a little girl, aged eight, who cried out and shouted with pain in the iliac fossa. She was placed under an anæsthetic, but the appendix could not be felt, or anything wrong detected on examination of the abdomen or rectum, so she was sent back 


\section{PERITONITIS}

to bed. Next day the pain was again severe, and after removal the appendix was found to be inflamed and surrounded by a localized peritonitis (not purulent). There was no concretion in the appendix, nor was it strictured. Here it would be well to state that in these cases very little, if any, benefit results from an examination under an anæsthetic when the periappendicular inflammation is becoming diffused.

Until we possess an instrument to measure pain, we must be cautious in estimating its value, for the power to bear it varies so immensely. There is nothing which relieves this pain so quickly as a hypodermic injection of morphia, but it should not be given without consideration, as its effect may be to mask symptoms and so prevent a due estimate of the severity of the case. The pain is generally accompanied by vomiting, and followed by a rise of temperature.

The amount of shock may be so great that the patient does not rally from it, dying in a few hours; or it may last for some days and then the patient gradually recover. In one case in which the patient suffered from an abscess which burst into the peritoneum the collapse was so great that for three days it was considered useless to take the temperature, as the patient appeared to be dying. When she ultimately rallied a large purulent collection was opened, and recovery followed. A diseased appendix was removed at a later date.

If the abdomen is examined early in the attack, the muscle will be found quite rigid and boardlike, and without movement on respiration. There is a protective contraction of the muscles best adapted to assist in the production of rest and to ward off injury from without. Sometimes the patient will attempt to take a full inspiration, but it is checked, the lower part of the abdomen not moving.

After a variable time this apparent contraction is succeeded by distension, due to intestinal paralysis and meteorism, and as this increases in extent the prognosis becomes more and 


\section{SURGERY OF THE APPENDIX}

more serious. This is especially so if vomiting is a persistent symptom. Distension with pyrexia and a history of sudden onset, may be taken as indicating peritonitis, which requires prompt surgical treatment.

During September, 1903, a case, seen with Drs. Hicks and Boden, gave a striking example of the importance of distension as proving the presence of peritonitis. The patient was a strong healthy man of forty-six, who had suffered from several attacks of abdominal colic, for which he had not found it necessary to call in a medical man, and when the present attack began he followed the same course as before-went to bed and used hot fomentations. As he was not better he consulted Dr. Boden on the second day. On the afternoon of the third day he had a sudden increase of pain and his temperature rose to $102^{\circ}$. The local signs were slight, and the pulse good. Twentyfour hours later, although his temperature was down, $99 \cdot 6^{\circ}$, pulse 60, full and strong, and the vomiting had ceased for the time, there was increasing distension and an anxious look. Operation showed a chronic inflammation of the appendix and a diffuse peritonitis, which proved fatal within three days.

Distension is a late symptom in intestinal obstruction from any cause, and should it suddenly arise in a patient the subject of malignant stricture almost certainly indicates a perforation. Occasionally the distension may be of a purely reflex character, as after abdominal injury or surgical operation for diverse conditions, but this is rare in our experience when there has been no actual exposure and injury of the bowel. A turpentine enema and a long tube should be used if there is any doubt or the amount of distension is causing discomfort. Should this distension subside and a swelling be felt in the right iliac fossa, the prognosis is good. If, on the contrary, little change takes place in the abdomen, it appears indeed to become more fixed, whilst the patient is anxious looking and restless, then the outlook is decidedly bad. 


\section{SPREADING PERITONITIS}

There are two kinds of oedema of the abdominal wall met with in peritonitis; one is the cedema which indicates the extension of a local inflammation directly through the structures of the wall to the skin and subcutaneous tissue. This is chiefly noticeable in cases of localized inflammation with suppuration. The second is a curious spreading œdema of the skin and subcutaneous tissues, which is of some importance but not often noted. Found only where the peritonitis is very intense, and associated with much subperitoneal exudation, the cedema spreads from the inguinal canals outwards, being limited in its course by the attachments of the deep layer of the superficial fascia in the same way as the spread of the fluid is controlled in cases of extravasation of urine. It passes into the scrotum and penis, but not into the thighs, and the invaded tissues are excessively sensitive to the touch. Since this condition was first described by one of us, Dr. Warmby Battle of Warrington, has kindly forwarded photographs which show clearly the appearance which this œedema produces on the surface of the body.

It is always some satisfaction to find an iliac swelling, because the diagnosis is thus rendered more certain; and it should be a routine procedure to make a rectal examination in all cases. But the surgeon will be guided by a careful weighing of all symptoms, and not by the presence or absence of any particular one in deciding on his course of action.

The importance of recognizing the earliest indications of spreading peritonitis due to disease of the appendix can scarcely be exaggerated. In quite a large number it is insidious in its onset. Few things in the life of the hospital surgeon give him more frequent cause for sadness than the hopeless cases of peritonitis which are brought for treatment, when all chance of relief is gone. It has been far too much the custom to blame the medical man in attendance, or the relatives, for the waste of time, but they are frequently only 


\section{SURGERY OF THE APPENDIX}

unfortunate and not deserving of censure. It is quite startling to see how very different from that of the onset of a typical case, is the inflammation in a class which is, we think, not uncommon. The history is the same in each whether given in hospital or private practice - that of a supposed attack of appendicitis of mild character, or a " simple stomach ache," and then a change for the worse or the rapid development of some unfavourable symptom, generally four or five days after the commencement of the illness. The abdominal wall is resistant, perhaps there is distension, but the important sign is dullness in the flanks and possibly above the pubes with a tympanitic note in front. This dullness implies the presence of a considerable quantity of free fluid, which shifts with the situation of the patient. Thrill is rarely found. If the abdomen is opened a large quantity of offensive semi-purulent or purulent fluid is evacuated, and a perforated or diseased appendix discovered; but whatever measures are combined with the operative treatment, life is rarely-very rarely-saved. The patient has absorbed a fatal dose of poison. We have specially drawn attention to this variety of peritonitis, because close attention should be given to the examination of all cases of abdominal inflammation from the earliest moment, to discover the presence or absence of this effusion.

The accounts of some cases seen in this early state are worth summarizing. Each was seized with abdominal pain and had a moderate elevation of temperature - in no case above $102^{\circ}-$ before operation. The pulse did not exceed 110, nor was the appearance of the patient indicative of serious abdominal trouble. Yet in each there was an exudation of considerable amount in the peritoneum within a few hours of the beginning of the illness; in one case the appendix was acutely inflamed, and contained pus, and was actually perforated in three others. Inflammation of the surrounding peritoneum had already commenced, and it is unlikely that it would have remained 


\section{INTRAPERITONEAL EXUDATION}

local. At operations within twenty-four hours of the commencement the fluid may not be offensive, but it contains abundance of the bacillus coli. If not evacuated it soon becomes offensive in smell and more purulent in appearance, whilst the deposit of lymph and formation of adhæsions between the coils of intestine limit its movement when the position of the patient is changed. The presence of free fluid is thus a fact of very great importance, and when found early in an attack of appendicitis is an indication of the presence of a grave lesion of the appendix, though it is of itself quite different in its effect to the sudden flooding of the peritoneum which follows rupture of an abscess, and is probably a protective exudation from the sub-peritoneal vessels. After a time, as the inflammatory changes spread in the parts surrounding the initial lesion, changes take place in the fluid itself, and the toxins produced by the bacilli growing in it are absorbed into the system. Ultimately, at a moment when things are apparently going on quietly and satisfactorily, a sudden change takes place, and the patient dies in a few hours. The dose of poison absorbed from the peritoneum has been too great. In some of these patients there has been very little sickness indeed, and until the final change there has been no symptom of sufficient gravity to attract special attention. We have known this dullness in the flanks to be mistaken for a loaded colon, and a loaded colon for the presence of fluid, also the change in note due to unusual muscular development has been ascribed also to the accumulation of fluid.

The first impression of a case is often important, for the expression of the patient may be a guide to the amount of the poisoning which has taken place. An anxious expression is often, in fact usually, seen in the early stage, but it is to the expression which comes later that we now refer. The face becomes flushed and drawn, whilst the eyes appear sunken and surrounded by dark rings. Sometimes it becomes purplish in 


\section{SURGERY OF THE APPENDIX}

colour, and the nostrils work quickly. We have known this appearance to be the only reliable evidence of the extent and gravity of the case, the local signs being entirely misleading.

As a rule early in an attack the tongue is white and thickly coated, and the breath foul ; later it becomes dry and brown, and the breath sometimes develops a sweetish smell. Yet in the early stage of many acute cases the tongue may be quite clean and moist. Vomiting is present with few exceptions in these attacks, but it may subside and not afterwards cause much anxiety ; if, however, the peritonitis is spreading, and has been left without operation, vomiting is certain to return, and is then a most serious sign, especially if the patient is at the time not taking anything by the mouth. Persistent vomiting is a very bad sign. When the vomiting is apparently effortless, simple pumping up, and the fluid brown and foul-smelling, the prognosis is most grave-such patients hardly ever recover. If the other symptoms indicate a grave amount of peritonitis, the surgeon must not hesitate, because vomiting is not for the moment a prominent symptom. For some hours before death it may entirely cease, the end being precipitated by the sudden expulsion of an enormous amount of material.

The bowels are not infrequently emptied soon after a perforation has occurred, and not uncommonly diarrhoea is present, especially in the more septic cases. Inaction of the bowel is the rule, but its relief is not often followed by much improvement.

The pulse may give warning of undoubted value, but sometimes it appears unaffected; in fact, we have known it quiet, full and steady on the third and even fourth days in fatal attacks of peritonitis.

A rapid pulse, one over 100, may be present in most inflammatory states, but if this pulse rate is exceeded and observations show that it is increasing in rapidity from hour to hour it is of grave import in peritonitis ; and if it becomes rapid 


\section{VALUE OF SYMPTOMS}

and small, out of proportion to the other symptoms, its warning must not be overlooked. Sometimes the appearance of the patient is calm, and excites no anxiety, but the rapid pulse and working nostrils give signals of danger not to be neglected. The heart muscle is affected by the absorbed toxins and it rapidly loses power, whilst to the running pulse is added a degree of breathlessness which is distressing, and a restless state difficult to control, and of grave significance.

All this with a clear intellect-in fact, the patient may be talking quietly at the moment of death-but is usually making some movement which throws extra strain on the heart, such as sitting up in bed, or striving to get out of it. A patient with general peritonitis with this restless condition should be as carefully watched and nursed as a patient in whom an attack of diphtheria is being treated.

We have already referred to the temperature in cases of appendicitis, and when this is complicated with spreading inflammation of the peritoneum the value of a temperature record varies very much. In a large number of the more acute cases the peritonitis is secondary to a gangrenous state of the appendix, a most acute septic process, and the variation in this condition is very great. Sometimes it is ushered in with a rigor and a high temperature ; at other times there is shock with a low temperature.

A most instructive and satisfactory case was one under the care of Mr. H. B. Shepherd-in this instance a youth of sixteen, who had had a slight attack of pain in the appendix region six months previously, was seized with a rigor in the evening, vomited, and complained of abdominal pain, not very acute. His temperature was found to be $106^{\circ}$. During the night the temperature came down, but in the early morning he had another rigor, and it again rose to $106^{\circ}$. The local and general signs independently of the temperature were not very marked, but sufficiently so to indicate inflammation in the appendix 


\section{SURGERY OF THE APPENDIX}

region, and to suggest that as the probable cause of the illness. The appendix was removed within twenty-four hours of the onset, found to be full of pus, with commencing gangrene of its walls, which were of very varying hue. There was early peritonitis in the parts adjacent and a considerable exudation of fluid in the pelvis, which was without odour and clear. $\mathrm{He}$ made an uninterrupted recovery.

Another instructive case was one under the care of Dr. Bowen Williams of Lewisham. A big stout man of forty-one had had symptoms of appendicitis for three days, and these of subacute character, with a temperature of $102^{\circ}$ on two occasions. When seen he was sitting before his fire, feeling much better, with a temperature of $99^{\circ}$, and not appreciating the severity of his illness-he was in the state of repose. His pulse was 80 and of fair strength, abdomen much distended without marked tenderness or rigidity of walls. Here there was a perforated appendix and diffuse peritonitis which proved fatal.

It is not necessary to give instances of a normal or subnormal temperature with suppurative peritonitis; the fact that it occurs is so well known that it is only necessary to warn a beginner against drawing a wrong conclusion from the fact. Most of us have had to meet the objection to a diagnosis of septic peritonitis, "but his temperature is normal," and then, " he does not look bad enough," when operation has revealed a hopeless condition of diffused suppuration. In many of these cases we get no history of the temperature before alarm was caused by the progress of the illness, and an opinion must be given on the state of things as found at the moment of examination.

In very rare instances a peritonitis of the septic kind, which up to a certain point was apparently spreading and likely to terminate fatally, has gradually subsided, and the patient recovered without proof of suppuration. The distension of the abdomen diminishes, the tenderness lessens, especially in 


\section{SUBSIDENCE OF PERITONITIS}

the upper part of the abdomen, the fever decreases and the pulse falls. Gradually the signs become more evident in the iliac region, then less troublesome and finally disappear. No surgeon would be justified in anticipating such a result in any case in which he had sufficient cause to diagnose peritonitis. We have seen two such cases, and been able to advise against immediate operation, because the symptoms were already improving when we saw them. It must not be forgotten that these are only two out of numerous others during a period of many years, and that the others all died where operation was declined.

Dr. Gerster (Annals of Surgery, vol. ii., 1893, p. 46) has published two cases, which were apparently in the last stage of illness from extensive peritoneal suppuration, when perforation into the rectum occurred, and recovery followed, but it is probable that these were really examples of large localized purulent collections. 


\section{SURGERY OF THE APPENDIX}

\section{CHAPTER VII}

\section{The Treatment of Diffuse Peritonitis}

\section{TREATMENT}

Is the treatment of peritonitis secondary to disease of the appendix, it must be remembered that we are not dealing with a localized suppurative condition in which the pus is confined by well defined boundaries. We are practically dealing with an abscess of indefinite extent and irregular outline, the contents of which are very poisonous if absorbed. The toxins developed in the cavity of the peritoneum from bacterial growth and changes are deadly, and much depends on the individual factor as regards power of resistance to their action; in some instances a few hours' absorption will be enough in which to ensure a fatal result, in others it is a question of days-absorption is less rapid, the poison less intense or the powers of resistance greater.

To mention two instances, both in young men, under the care of one of us at St. Thomas' Hospital, admitted during the same week, with evidences of acute localized spreading peritonitis of almost equal duration and extent. The treatment of both was similar : incision in the iliac region, removal of the appendix, careful flushing with sterilized saline solution, followed by gauze and tube drainage. In one, improvement was only temporary; he became extremely restless and excited with a rapid pulse, which was uncountable before death. In the other, who suffered much more from shock as a result of the operation, and only revived after saline infusion into the median basilic vein, recovery ultimately 


\section{DIFFUSE PERITONITIS}

resulted, but for some weeks, long after the abdominal trouble was over, there was an abnormally high pulse rate. In both of these patients the local extension of the disease was arrested by the treatment. Yet at the time of the operation it was thought that the case which ultimately proved fatal would do the better of the two.

Diffuse septic peritonitis due to appendix disease is usually an inflammation of an unchanged peritoneum, one with all its powers of absorption unimpaired, and its nerve endings acutely sensitive to stimuli. There can be, therefore, no surprise felt at the extreme gravity and high mortality of this disease.

In cases of the fulminating type it is bad to put off any surgical treatment in the hope that the patient will perhaps rally sufficiently to permit of operation later on. The condition is probably a mixture of shock and the result of absorption of toxins, and if the treatment of the shock be prolonged unduly, the patient will die from the poisoning. The prompt administration of a saline infusion, with rapid operation under ether as an anæsthetic, gives in many instances the only chance of saving life.

Some confusion has arisen in the past from the grouping of all forms of spreading peritonitis due to disease of the appendix under one heading. At St. Thomas', from a clinical point of view, it has long been recognized that this is not correct, and the disease is divided for statistical purposes into acute localized spreading peritonitis and general peritonitis. They are only stages of the same disease, but the result of operation in the two is very different, and if they are grouped under the one heading, that of acute peritonitis, the result of treatment of the more serious condition appears far better in one hospital than in another, or in the hands of some one operator, than is really the case.

The principles of treatment are clear, but the best way of 


\section{SURGERY OF THE APPENDIX}

carrying them out is still a subject of discussion. They are as follows :-

1. To open the abdomen in such a manner that the area affected can be thoroughly treated without undue handling.

2. To evacuate the pus and cleanse the peritoneum.

3. To remove the diseased appendix.

4. To provide adequate drainage.

5. To combat shock during the operation and afterwards.

6. To counteract the effects of the absorbed toxins.

It may be said, in the first place, that rapidity in operating is most important in these cases; the shorter the time during which the patient is exposed to adverse influences on the operation table the better. Much of the success of any operator will depend on his power of operating quickly on a certain definite plan, and on the efficiency of his assistants. Nothing is much more unsatisfactory than the hurried operation with repeated and inadequate examination of parts. Fvery time the gut is pulled into the wound when acutely inflamed further injury is done to it, and a fraction, not easily estimated, taken from the patient's chance of recovery.

The surface of the abdomen can only be properly cleansed after the anæsthetic has been administered, and care should be taken that no undue pressure is made in the process or useful limiting adhesions may be ruptured, and the pus be diffused into parts previously free from invasion.

The choice of anoesthetic is an important one. Shall chloroform or ether (preceded by gas) be given? We are in favour of the latter in those cases where the general condition is good, the disease localized, or where there is a tendency to cardiac failure, whilst if there appears to be any congestion of the lungs, or marked respiratory difficulty, the former would be advisable.

One argument in favour of the employment of chloroform 


\section{TREATMENT OF PERITONITIS}

instead of ether, is that the latter is much more likely to cause a cough to develop, which may be of very serious moment during the after treatment. Should the wound become septic (and this is not unlikely), much difficulty might be experienced in preventing the stitches from giving or tearing out, and this accident would permit of a protrusion of abdominal contents should the patient cough. The patient might recover but the process of recovery would be decidedly unpleasant and the management of the case tax the ingenuity both of the surgeon and the nurse.

In some, local anæsthesia might be a feasible means of preventing pain during the operation, and Dr. McCrae speaks highly of the use of cocaine in such cases as perforation of gastric ulcer, when of course the state of the patient is often as desperate.

Another very important question to decide in these cases is the time at which it is best to operate--and there is no class of disease amenable to surgical treatment in which the surgeon has more decided evidence of the value of early operation than he has here in cases of peritonitis due to disease of the appendix. In support of this statement we give below the table which was compiled by Dr. Berg.

The relation of cures after operation according to the previous duration of illness :-

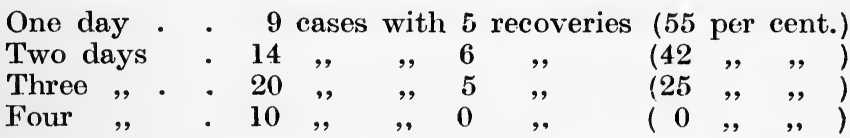

We have obtained successful results after operation at St. Thomas' Hospital later than this, even in most unpromising cases, but these are almost surgical curiosities, and the mere fact that it is ever necessary to operate at so late a stage implies discredit somewhere.

The earlier the operation the better the chance of the 


\section{SURGERY OF THE APPENDIX}

patient when suffering from this complication of appendicitis - a statement which applies also to perforations of the stomach and intestinal tract generally.

Incision.-There has been a difference of opinion as to the best line of incision to adopt in these cases : Should the surgeon open the abdomen in the median line below the umbilicus, or make his incision in the appendix region? We think the answer to this question should depend on the particular case requiring operation. If the inflammation has undoubtedly arisen from the appendix, and is still comparatively localized, then incision in that region will probably admit of quicker manipulation of parts diseased than one in the middle linethe appendix will be more easily reached and removed, and the region in which there is the greatest change will be under the direct examination of the operator. We consider that the best incision in this part is one through the rectus muscle, its fibres being separated but not in any way divided. These wounds nearly always become septic; if they do, there is a very great probability that a hernial protrusion will afterwards develop through the scar. If the incision is made through the muscle with efficient after-drainage this suppuration should be confined to the track of the tube; should hernia follow later the muscle can be repaired and united with sutures, and so a firm barrier placed to prevent further protrusion. There is the chance that suppuration may follow in the sheath of the rectus, and we would not minimize the danger to which that gives rise, but think that with care serious results may be prevented. One great advantage is that the wound can be quickly made any requisite length without increase of risk. An incision through the linea semilunaris is apt to gape widely if the stitches give, from traction of the muscles to the outer side, and whilst a cough would tend to bring the muscle edges together in the rectus incision, it would cause further dangerous opening or dragging 
on the edges of the wound in the other. A long incision through all muscular tissues would be very easily made, but the ultimate results would be very troublesome, from weakness of the abdominal wall.

Should the peritonitis be general, thus rendering it necessary to treat the whole of the peritoneal cavity, or should the case be a doubtful one, then a median incision should be made. Sometimes it is not possible to remove the appendix from the middle line; an incision should then be rapidly made over it, and any drainage carried out through the median incision only.

The method of making many incisions, over the pubes, in the iliac regions, and in the loins, does not commend itself. No better result is obtained and success does not depend so much on the after-drainage as on the perfection of the cleansing of the peritoneum done at the time.

The difficulty of operating will be very much increased if the intestines are distended and the abdomen large, as in the middle-aged adult. There is hardly anything which interferes so seriously with the operation than a thick fat abdominal wall. Where the intestine is distended the nearest coil should at once be brought out of the wound, surrounded with gauze and punctured with a trochar and cannula, or knife, the contents being received in suitable sterilized porringers or other vessels. The puncture with a knife enables the emptying of the gut to be done more quickly, whilst the bowel is more thoroughly under control. The surgeon knows how often a cannula slips out and fouls surrounding parts; theoretically it conveys intestinal contents to the porringer with safety, but practically it acts unsatisfactorily. An assistant should empty neighbouring coils into the section held; after this the operation should be repeated with other coils, as may seem best suited to the rapid emptying of the gut. The openings left after the punctures must be closed with a 


\section{SURGERY OF THE APPENDIX}

Lembert suture or two, and the gut cleansed before it is put back again. Some operators have taken advantage of the puncture and whilst the cannula was still in position have introduced sulphate of magnesia into the bowel. The value of the injection of aperients such as sulphate of magnesia into the bowel is not easy to decide. The salts are unfortunately injected in a concentrated form and not with a large quantity of water, when they act best. Forty-eight hours usually elapse before any evacuation occurs, and no action happens in the very instances in which there is most need. Shortly, the patient loses nothing by this procedure, and it may help to save a few doubtful or borderline cases.

It is not much use trying to cleanse the peritoneal cavity until the more distended coils have been emptied, for there is no room for movement of the hand, and if any sections of distended bowel escape without having been emptied, it may be impossible to bring the edges of the wound together later, or to reduce the escaped intestine.

A useful method of replacing the bowel which has been examined is to cover it with a thin layer of a long strip of gauze, allowing the gauze to enter the peritoneal cavity with the loop as it is returned. There is less injury to the peritoneal covering of the gut and the gauze takes up some of the peritoneal fluid, and brings it away when it is extracted towards the end of the operation.

In order to evacuate the pus and cleanse the peritoneum, it is necessary to have an opening of adequate size, that is, one through which it is possible to carry out the requisite manipulations without bruising of the edges of the wound, or the bowel which may require to be manipulated. It will save much time if this opening is at once made large enough to admit the hand of the operator. Much of the pus will be found in the pelvis and right flank, but it may also extend upwards to the liver on the right and the spleen on the 


\section{TREATMENT OF PERITONITIS}

left side, and some will make its escape during the early examination after the peritoneum has been opened. Probably very offensive, its amount varies in each case, and often when the surgeon thinks he has evacuated the full amount, a movement of the finger opens up some new loculus. We have already given advice as to the wearing of rubber gloves in these cases, and need not do more than remind our readers of it. They are apt to slip when it is necessary to draw intestine to the surface; but if gauze is used and interposed, this difficulty will be overcome. As a rule the marine sponge has been replaced by a sterilized one of absorbent wool in gauze, and this is light, yet effective; we think also that it acts better in separating adherent lymph. Some surgeons are satisfied with dry sponging, and do not use any solution to wash and cleanse the parts. This may be effective when the inflammation is localized and not very intense, but when it is extensive and there is much pus, we consider it better to use quantities of saline solution and thoroughly wash out the whole peritoneal cavity. We cannot approve of dry sponging of the peritoneum, as we hold it more likely to cause adhesions subsequently than if saline fluid is used. When it is desired to cause adhæsions in the peritoneal cavity in cases of ascites due to portal obstruction we employ a dry sponge. Occasionally it may be necessary to bring the whole of the intestines outside, performing an exvisceration, as it is called. Sometimes this method is most clearly the best to employ, and we fear has obtained unfair consideration because of the name applied to it. The advocate of exvisceration is rather held up as a kind of vivisector who does a dreadful thing. Now there can be no question that operation in these cases should be quickly performed, and if there is plenty of assistance and warm saline fluid, the parts may be cleansed efficiently in far less time than by pulling up the intestine and wiping it in sections; and we maintain that the shock is often less than 


\section{SURGERY OF THE APPENDIX}

that produced by the more prolonged operation. Too much time must not be expended in trying to wipe off lymph, especially if very adherent, and its removal causes bleeding from the surface to which it is attached, it is not necessary; but it is unwise, if it can be avoided, to leave any thick flakes, as they will prove convenient nests for the development of bacteria. We think the best plan is to keep a gentle stream of saline fluid (temp. $105^{\circ}$ ) flowing over the wound and exposed peritoneum during the manipulations, either from the end of a rubber tube connected with an irrigator, or by means of a gentle stream from a jug. Some of this fluid may be left in the peritoneum, but irrigation should be continued until the outflow comes away clear and without unpleasant odour.

The removal of the appendix should always be carried out as the first part of the operation and that by some method which is found efficient at other times, i.e., when no peritonitis is present. For instance, if the clamp and ligature be used, the stump must be sutured into the cæcum. If this is not done a fæcal fistula will almost certainly result, and may cause much trouble for a time in the later treatment. If the condition of the patient is, however, very bad, and the necessary delay likely to be very dangerous, a single ligature may be applied, but it is not a good practice, and only emergency can excuse it.

Cases of general peritonitis may get well even though the appendix be left in the peritoneum, but a continued infection must go on from it for a varying period and it will require removal later on.

After operation for peritonitis following disease of the appendix drainage should always be employed, and this may be done by means of a Keith's glass tube or a perforated rubber tube. Patients have recovered when the wound has been closed, but we are convinced that it is much more safe to drain the peritoneum, and that some might have recovered with drainage 
who did not do so when the wound was closed. The tube should be passed into the pelvis because this is the most difficult part to cleanse, and much of the affected bowel dips into it. We do not think that strips of gauze placed in the peritoneum are enough, as already explained, to carry off superfluous fluids, but placed around the tube, extending in different directions amongst the intestines, they undoubtedly take up a good deal of the earlier exudation from the intestinal wall, which is favourable to abundant bacterial growth. These plugs should be removed at the end of fortyeight hours, and an anæsthetic given whilst this is done and the dressing changed. Should a stitch have been left loose in order to admit of the passage of the plugs it may now be tied. Sometimes the condition of these subjects is so bad that the surgeon has no time to introduce sutures to close the wound; it nust be packed with gauze round the tube and the abdomen firmly bandaged in the hope that a rally may be made from the state of collapse, and the operation completed in a second stage. We are in favour of a mild antiseptic in the gauze used for plugging or drainage, as septic changes in the fluid and surrounding parts are less likely to progress in its meshes.

Antiseptic injections may also be required if the fluid which comes away from the tube is foul-smelling, but for ordinary syringing just to cleanse the tube during the time it is kept in, saline fluid usually suffices.

The usual methods to guard against shock should be taken, The arms and legs wrapped in flannel bandages, hot water bottles placed near the patient, and a hot water cushion under his back, that is, if it is not possible to get a hot water table, or one heated with electricity. Before the operation is commenced a warm brandy enema should be administered. During the progress of the operation everything should be ready for the administration of a warm saline infusion; this 
is not infrequently required, and should be given directly into a vein, median basilic of arm, or internal saphenous of leg. This solution should consist of one drachm of salt to a pint of sterilized water, and be passed slowly into the vein by elevation of the tube and glass funnel. Some operators combine brandy with the solution. It is not surprising to find the effect of this infusion to be so excellent when we recollect that in addition to the shock of the operation the patient is more or less "dehydrated" by frequent vomiting of fluids which it has not been possible to replace, excepting by absorption from the tissues. Subcutaneous injections of ether and of liquor strychnix- $m \mathrm{v}$.- (or even $m \mathrm{x}$.) are useful in extreme shock.

After operation, the lower end of the bed should be raised on blocks, and the patient wrapped in warm blankets outside which, but not pressing closely to the body, should be hot bricks wrapped in flannel or hot water bottles. A small brandy enema should be given at once, and for some hours enemata of saline at intervals, varying with the state of the patient. At first only teaspoonfuls of fluid should be administered by the mouth every halfhour if awake, but as the tendency to sickness passes off this may be increased.

As the saline infusion supplies fluid which has been lost to the body, as a result of the vomiting and possibly diarrhœa, so it prevents the necessity of further absorption, at the same rate as before, of fluid from the tissues to replace that lost, especially from the abdominal organs, which are more or less impregnated with toxin. Sometimes the vomiting will continue for a few hours as a result of the anæsthetic, at other times as a result of extension of the inflammation in the abdomen, and possibly as a result of the action of the absorbed poison. Here it is advisable to continue the supply of saline fluid over a period of time, as then it is very much needed. Enemata are useful, but a con- 


\section{TREATMENT OF PERITONITIS}

tinuous steady additional supply is required. For this purpose the plan of Kocher has great advantages. A cannula is placed in the subcutaneous tissue on the inner side of the thigh, and warm saline fluid allowed to pass slowly hour after hourthe cannula being moved to the other side of the body or to the axilla, if the skin has become sore where it was first placed. This of course requires the careful attention of a good nurse. The sudden injection under the skin of larger quantities of fluid saline, does not appear to have so satisfactory a result.

An early evacuation of the bowels is to be desired, and the administration of a saline purgative on the second day will usually prove efficient, a drachm of sulphate of soda, or the same quantity of sulphate of magnesia, repeated in three hours' time if necessary.

These patients require the most careful watching, and if there is much restlessness and a rapid pulse, injections at intervals of two hours, or even less, of liquor strychniæ must be given. If possible no morphia should be administered during the after treatment, unless it is absolutely necessary to quiet the patient or produce sleep.

A judicious stimulation is usually required.

Later all patients should be supplied with an abdominal belt and made to wear it regularly, and in some an operation may be required to restore the weakened abdominal wall. 


\section{SURGERY OF THE APPENDIX}

\section{CHAPTER VIII}

The Diagnosis of the Acute Abdominal Conditions which Require Surgical Treatment

\section{THE DIFFERENTIAL DIAGNOSIS OF ACUTE APPENDICITIS WITH PERITONITIS}

The differential diagnosis of acute appendicitis may be one of the most difficult of the diagnoses that a medical man may be called upon to make. The importance of his decision to the patient, and the soundness of the line of treatment adopted in consequence, are matters which frequently entail the life or death of his patient. Too much stress cannot be laid upon the subject under consideration. Fortunately the treatment of the majority of cases is much more easily decided on than the diagnosis can be. The most momentous question for the life of the patient is the recommendation, or not, of operation. As will be shown later, the adoption of this course when the peritonitis has been permitted to become diffuse has changed the mortality from almost nil in the former to 84 per cent. in the latter. The very ease with which this line of treatment can be advised implies that the decision should have been made earlier. The great gravity of the cases, the great desirability of the early recommendation and adoption of a line of treatment makes it imperative that the subject of the diagnosis should be made as clear as possible. To gain this end the following plan has been adopted with the consequent sacrifice of such details and cases as have 


\section{DIFFERENTIAL DIAGNOSIS.}

been found to obscure the subject in many monographs. The matter will be discussed under these headings :--

1. The importance of appendicitis in relation to acute abdominal disease in general.

2. (a) A general description of the "acute abdomen."

(b) Special points to be watched, which experience has shown to be the most trustworthy.

3. The differential diagnosis of cases not demanding immediate operation.

4. The recognition and importance of appendicitis with local spreading peritonitis.

5. The diagnosis of the cases demanding immediate operation.

6. Cases in which a diagnosis is impossible.

In order to obtain practical and clear ideas of the relative frequency of the various factors in the causation of acute abdominal disease, Dr. A. E. Russell and one of us have recorded the acute abdominal cases admitted to St. Thomas' Hospital during the years 1900, 1901 and 1902. In all, there were 456 cases, of which 168 , or 37 per cent., arose from disease of the appendix, forming the largest factor by far in this class of disease.

Acute Abdominal Cases.

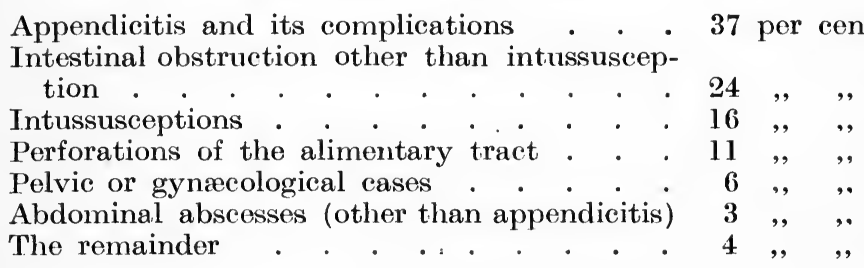

The enormous importance of the rôle which the appendix plays is clearly shown by this table. And it is still further emphasized by the fact that the mortality throughout was 56.5 per cent. The first four in this list form the great classes 


\section{SURGERY OF THE APPENDIX}

and also fall into age-epochs. Thus, intussusceptions are diseases almost entirely of the first year or years of life ; acute appendix trouble is most common by a long way between the ages of ten and thirty; alimentary perforations between the ages of fifteen and forty; intestinal obstruction (other than by intussusception) from the age of thirty onwards, with increasing frequency to a maximum between fifty and sixty. It will thus be seen that the age of the patient will give some probability as to the origin of the disease.

Before proceeding to the differential diagnosis of the cases it will be as well to mention the varieties of the disease of the appendix recognized in these cases. Of the 168 cases ( 37 per cent. of all)-

Appendicitis and local spreading peritonitis formed . . . . . . . . . . 18 per cent.

Appendicitis and local abscess formed . . 23, , , , and diffuse peritonitis . . . $59,$, ,

The most important lesson that study of these classes has shown us is in their relative mortalities. For the first two the death rate was nil, and that of the third 84 per cent. Nothing can show the evils of delay and procrastination as regards operation more than these figures. The terrible truth that they teach should be borne in mind in every case of appendicitis, especially those with acute onset.

The analysis with regard to sex is also of some interest.

In cases of local abscess the proportion of males to females is practically equal, or 5:5.

In cases of local spreading peritonitis the ratio is $5: 3$.

In cases of diffuse spreading peritonitis the ratio is $5: 1$.

The preponderance of males is in that subdivision of the disease which has the enormous mortality of 84 per cent.

(I.) A GENERAL DESCRIPTION OF THE "ACUTE ABDOMEN."

It is by no means easy to give a concise account of the clinical appearance of these cases. The general symptoms are 


\section{THE "ACUTE ABDOMEN"}

those of a person who is really ill, unless these are hidden by the injudicious administration of morphia. The face is almost always anxious-looking. The pulse is increased in rapidity, the respirations are slightly more frequent than normal, and shallower, the respiratory movements being chiefly costal. The abdomen, to which the attention is mainly called, is distended, more or less motionless, tender on palpation, sometimes also on percussion, and often presents the signs of free fluid, such as dullness in the flanks, which shifts on movement. Vomiting is almost invariably present and after that of the onset may pass off a little only to become distressing later. The tongue is always furred, and the breath often foul, though in cases of chronic disease on which an acute attack has been superimposed, a sweet and sickly smell may be noticed. The bowels are almost invariably without action both to frees and flatus. A wrong impression is created by the use of the word constipation in this sense, for the case is rather one of inaction of the bowel. In many cases the onset of the attack is accompanied by a diarrhœa, often offensive ; or a looseness of the bowels may be a late symptom, and is then called "septic diarrhœa." But there is always some obstruction to the passage of the contents of the alimentary canal, either by a growth, volvulus, band, segment or segments of paralysed gut, etc. A rectal examination should always be made, though in the majority of cases it yields a negative result. A further general sign which may often be observed in these instances is dilatation of the stomach. It is not usually an early, but rather a late sign, and depends partly on the general toxic condition and on the growth of organisms within it in consequence of the disordered condition of the digestive tract. The urine is usually scanty, at first normal, later it may contain albumen, but rarely blood.

The above description will appear to many short and perhaps inadequate, but it is very difficult to avoid obscuring 


\section{SURGERY OF THE APPENDIX}

the picture with details. Variations are naturally seen, often according as to the causation of the disease. Consideration of these variations constitutes the differentiation of the diagnosis and will be considered then. But the picture varies also with the individual and presumably the character of the infection. Thus a man with a perforated duodenal ulcer had a pulse of 80 , and a girl with a suspected perforation of a gastric ulcer (really non-existent) a pulse of 120. The temperature is nearly as often raised or depressed as normal. So that in one case, the general symptoms predominate and action must be taken on them; in another case, local signs predominate and the patient's general condition is good. It is the latter which constitutes the difficult position for the practitioner, and the question arises, is he to believe himself or the patient who says that he is "all right."

Thus the real state of the case often escapes his attention until too late to save life.

\section{(II.) SIgNS AND SYMPtOMS ESPECIALLY TO BE WATCHED.}

It has been our endeavour to select such indications as experience has taught us to believe to be most reliable. A lengthy discussion of the points, and other views, has been avoided, as such would defeat our object, namely to put the material as clearly and concisely as is possible.

At the onset, cases which begin with severe constitutional symptoms, and especially so if the abdominal signs are not equally prominent, should raise the suspicion of some acute infection with severe intoxication. Delay of operation in such cases is very fatal ; a sharp rise of temperature, especially if it is accompanied by a rigor, may mean little or indicate some severe disturbance, and the case must be watched carefully at short intervals. Diarrhœa, when present, may be regarded as of septic origin, and, therefore, indicative of a 


\section{THE “ACUTE ABDOMEN"}

more serious condition. The presence of blood in the stools or the vomit, such as "coffee grounds," are warnings that should never fail to raise suspicions. Cases in which the abdominal symptoms are most marked do not need special mention, as they declare themselves.

It must be borne in mind that the onset of the disease sometimes does not give any indication as to its subsequent course, and that the severest symptoms may arise in apparently the mildest cases. This happens much more often than that those with severe onset quieten down.

In estimating the condition and the changes which occur therein, attention is drawn to the following points :-

1. The Pulse.-Although it is but rarely that a patient's normal pulse rate is known, yet, after the onset, generally speaking, a patient with a pulse over 100 should be carefully watched and if the rate of beat reaches 115-120, in all probability operation is urgently called for. Some authors have modestly expressed their inability to appreciate the quality of the pulse and its relation to disease, yet changes in its quality must be watched, and we think should be correctly estimated by a doctor in charge of such a case. Surely every surgeon must have been aided in his opinions of abdominal cases by the character of the pulse! We have not attained the excellence by which small pathological changes can be estimated by us in the quality of the pulse. Yet our observations are such as to be of the greatest value in clinical work, which is in reality coarse as compared with pathological.

2. The Temperature is often most misleading, especially at first. Later, its variations become more sure expressions of the patient's condition. It should begin to fall on the second day. But as it may remain normal, or be subnormal, it should be read in conjunction with observations on the pulse. For instance, a subnormal temperature and a rapid pulse is "outspoken." 


\section{SURGERY OF THE APPENDIX}

3. Vomiting, after the onset, should cease; and its persistence rather than its severity is the more important in helping to form an idea of the pathological processes "going on inside." Vomiting is usually the most severe in first attacks, and it is almost always first attacks that lead to diffuse peritonitis.

4. After the above, the progress of the abdominal signs is most important, and special reliance must be laid on repeated rectal examinations. The onset of meteorism, increase in the area of rigidity, dullness, or tenderness, indicates an increase of the area involved by the peritonitis ; and this may be further emphasized if rectal examination for the first time reveals tenderness.

Changes in general condition and other points are a great help in forming an opinion of these cases. In many instances when all are reviewed together it becomes clear whether operation should be performed at once, or if further watching is justifiable. It may also be added, that any tendency to error should be on the side of recommending operation in acute cases, for it is far more dangerous to watch than to operate on them. Clinical manifestations are not completely understood, and always are seen later than the pathological changes which cause them.

III. THE DIFFERENTIAI DIAGNOSIS OF CASES THAT DO NOT DEMAND IMMEDIATE OPERATION.

In the first place, at the onset of all acute abdominal disease, whether it is the perforation of a gastric ulcer, volvulus of the intestine, etc., there are similar symptoms constituting what is known as "peritonism," i.e. shock, abdominal pain, vomiting, etc: Therefore from the signs found early in a case, no definite opinion of the cause can be formed. But the history of the case may guide; for instance, if a patient with a gastric ulcer or typhoid fever has an attack of abdominal pain with 
collapse, etc., there is reason to suspect that in reality it is a case of perforation and not of appendicitis. In such cases action can be taken at once, though it may be advisable to see the patient again in a few hours' time. Except as regards the history, it cannot be expected that anything definite can be found to point to the site of the lesion within the first twelve hours. And before recommending operation it is desirable that the practitioner should first endeavour to exclude all possible causes of confusion in which operation is not needed. With increased frequency of operation this has become of considerable importance, and a word of warning may be given at once. Although it is the abdomen and the abdominal signs which thrust themselves upon the notice, care must be taken to look further afield and especially to the condition of the thoracic viscera.

This group of cases is large and, like most things which are slowly becoming recognized, is growing.

One of the most common is colic; when this occurs in nervous people it may present features extremely like those of the "acute abdomen." The greatest difficulty is experienced sometimes in differentiating the two. An opinion can only be formed as the result of two or three examinations of the signs and symptoms. The administration of opium causes the greatest confusion in these cases, though if the diagnosis is sure it is a reasonable treatment. But it masks the symptoms so completely that on the one hand, perforations, etc., have been overlooked, important signs being suppressed, and on the other, healthy abdomina have been opened as seemed justified by the history and the fact that opium had been given freely, which prevented a true opinion from being formed of the local condition. When the colic has been caused by lead the blue line is found on the gums. But a similar discoloration is often seen in badly kept mouths. Again, let it be pointed out that these conditions have been 


\section{SURGERY OF THE APPENDIX}

known to coexist, e.g. appendicitis with peritonitis in a person subject to lead colic.

In rare instances acute indigestion may bring about a clinical picture somewhat like that of the "acute abdomen." The amelioration of the symptoms, the almost invariable history of a dietetic indiscretion, the relation of the taking of food to the vomiting, etc., all give clues as to the possible source of the trouble. But it must be remembered, as has been pointed out in the account of " apparently non-suppurative" appendicitis, the premonitory and initial symptoms are those associated with an ordinary bilious attack. It is, therefore, clear that only by carefully following the course of the signs and the symptoms of the case can the correct line of treatment be taken. The same will apply to ptomaine poisoning, acute gastro-enteritis and like alimentary disturbances.

In the course of typhoid fever and gastric ulcer crises may be noticed in which a call may be made for decision as to whether the case is one for operation or not. In the former, the cause of these attacks is believed to be some small perforation that has become plugged by its own mucous membrane, the omentum or contiguous coils of bowel. In the latter, the association of it with gastro-intestinal disturbances generally renders these attacks most intelligible as colic. In both, reliance must be placed on the pulse, the persistence of vomiting, if it is present, the progress of the abdominal pain, signs, etc. The most difficult of complications to exclude in typhoid fever is that of hæmorrhage into the bowel. In both this and perforation you have shock and collapse, abdominal pain, and perhaps vomiting, with a fall of temperature. One sign, which Dr. A. E. Russell pointed out in a discussion at the Clinical Society in 1902, is the progressive obliteration of the liver dullness in cases with undistended or even retracted abdominal walls. This sign is of great value, and in his hands 


\section{THE "ACUTE ABDOMEN"}

and those of Dr. Singer we have known it to lead to the correct diagnosis of intestinal perforation in typhoid fever within two to nine hours of the occurrence and before the fall in temperature was apparent. It may be added that in two of these cases, under the care of Dr. Hector Mackenzie, ${ }^{1}$ operation was successfully performed by one of us.

In dysentery and colitis attacks of abdominal pain occur which somewhat resemble a perforation. These generally mean nothing requiring urgent interference. Colitis in some of the neurasthenic cases sometimes gives rise to such intense abdominal pain as to cause almost maniacal outbursts.

Biliary colic occurs most commonly in women and in both sexes at an age after the period when appendicitis is most common, (i.e., between ten and thirty). Some cases are difficult to distinguish, especially when it is remembered that appendicular pain is not infrequently referred to the region of the gall bladder. The vomiting as well as the colic may be relieved, and often stopped, if the stomach is freely washed out with warm water. Cholecystitis and cholangitis are far from easily diagnosed in themselves, let alone their rare interference in the diagnosis of appendicitis. Whilst considering these hepatic factors in the diagnosis. mention may be made of embolism of the portal vein, pylephlebitis or inflammation of the portal vein, its tributaries, and their thrombosis. These are frequently septic complications of appendicitis.

The renal factors which enter into the subject under discussion are movable kidney, torsion of the vessels and nerves of the hilus and the ureter, renal colic, and such rare things as rupture of a hydronephrosis, etc. The movable kidney which gives rise to these attacks of pain is found in women, and frequently not long after a pregnancy. The pain is associated with some torsion of the pedicle. As these kidneys

${ }^{1}$ Lancet, October 10, 1903. 


\section{SURGERY OF THE APPENDIX}

are often displaced they may be found in the right iliac fossa or the pelvis, still further simulating acute appendicitis. When discussing the diagnosis of appendicitis alone and apparently uncomplicated, special mention and stress was laid upon renal colic, and particularly so in the connexion of pains which arise from movements of a stone in the pelvis of the kidney. In connexion with the diagnosis of appendicitis with peritonitis, pain associated with the passage of a stone down the ureter is of most importance. Attention to the character, the progress and extent of the pain, the condition of the urine, etc., will serve as the best guides to the recognition of the source. As was mentioned amongst the diseases connected with the liver which entered into the diagnosis of acute appendicitis, sometimes symptoms of the latter disease were referred to the gall bladder region; this is true in respect to the kidney. Renal irritation is sometimes seen with pain, frequent micturition, perhaps hæmaturia, albuminuria or pyuria. A concomitant inflammation of the ureter, ureteritis, may give rise to intense pain during the passage of urine through the inflamed segment, a condition like to renal colic. But these are rather complications of appendicitis, though their presence without it may be borne in mind.

Dr. James Mackenzie, in the British Medical Journal, 1903, ii. p. 66, pointed out that certain bladder symptoms may mask the diagnosis of appendicitis, and that these are of two kinds. The first consists of a reflex frequency of micturition, the act being unimpeded. The second is associated with spasm of the sphincter vesicæ and exhibits both frequency and difficulty in micturition.

The pelvic organs enter very largely into the diagnosis of appendicitis especially so as the latter frequently infects the pelvis. And in point of fact, as has been said when considering the pelvic complications of appendicitis, the exact interrelation of these two sets of diseases is not completely under- 


\section{THE "ACUTE ABDOMEN"}

stood. Disease of the internal generative organs of the female does not often give rise to acute general peritonitis, but rather to a subacute or chronic form. One condition may be especially mentioned which does not demand operation; this is the association of dysmenorrhæa with a bighly nervous temperament. The picture of peritonitis may be excellently reproduced. Far more common is the salpingitis and pelvic peritonitis which does not demand operation. Metritis and cystitis may be so acute as to occasionally confuse the diagnosis.

One of the most important subjects to which attention should be directed and which must be excluded, is the simulation of acute peritonitis by diseases of the thoracic viscera. Dr. A. E. Russell and one of us made extensive observations on this subject at St. Thomas' Hospital during the years 1901 and 1902. In the majority of cases it is only necessary that suspicions of the state of affairs should be aroused and the mind rendered alert for the differentiation to become easy. We observed this complication in pneumonia, especially of the right base, pleuro-pneumonia, diaphragmatic pleurisy, pleurisy, pneumo-thorax, pyopneumothorax; etc. Cardiac and pericardial diseases do not seem to cause this. Andral, as long ago as 1836, first drew attention to the similarity between the signs which may arise from thoracic and abdominal diseases. Pathologically the conditions arise from irritation of the last six dorsal nerves, the tenth and eleventh on the right side specially causing the simulation of appendicitis, and reflexly referred visceral pain along the seventh, eighth, and ninth dorsal nerves ${ }^{1}$ the visceral signs perhaps are due to irritation of the splanchnic nerves derived from the lower six dorsal, or directly of the trunks of the splanchnics. The abdominal signs and symptoms may consequently be found both in the parieties and viscera. The diagnosis is usually easy if sus-

1 Dr. Henry Head, Brain, 1896. 


\section{SURGERY OF THE APPENDIX}

picions are aroused as to the state of affairs. The most obvious thing is the rapidity of the respirations, over forty a minute. The pulse is usually not raised correspondingly in rate. The abdomen is rigid and tender, but may be noticed to become soft and unresisting for a few seconds every now and then, especially when the patient answers a question. Again, there are no localizing signs such as dullness, etc. And what seems a most important sign, the abdomen is more tender on palpation than percussion, which is usually the opposite in peritonitis. Firm deep palpation is often found to be pernitted. The temperature is high, say $103^{\circ}$, which it is not often in acute appendicitis with diffuse peritonitis. The sickness, abdominal distension and constipation may be the same in each set of cases. If attention is turned to the thorax no physical signs may be as yet discoverable, and they will only appear later.

In acute hæmorrhagic pancreatitis the patient will lie on either side, according to his desire to change position, whereas in appendicitis with peritonitis he will remain on the back with flexed thighs and legs.

\section{APPENDICITIS WITH LOCAL SPREADING PERITONITIS.}

The difference between appendicitis with local spreading peritonitis or diffuse peritonitis is purely one of degree. But the subject of its clinical recognition is of prime importance. As has been said already, the St. Thomas' Hospital figures for 1900, 1901, and 1902 have shown that operation when the peritonitis is localized to the iliac fossa had no mortality in some forty cases, whilst when the peritonitis was diffused the mortality rose to 84 per cent. The figures show clearly the large number of lives which were sacrificed by the non-recognition and proper treatment of the nature of the trouble early. It is in the power of medical men, if only the cases come under their care soon enough, to save a 
large number of most valuable lives. Recently published statistics of cases of recovery after appendicitis and "general peritonitis," and so forth, have derived their pleasing characters from the inclusion within them of cases with as yet but local peritonitis. The non-existence of adhæsions was held to indicate the general character of the inflammation. The appreciation of the pathological state, followed by the clinical recognition of the phase of local (but not localized) mischief should be followed by a remarkable decrease in the mortality, and the greatly improved results will reflect more credit on the diagnostician than has hitherto been given him. With the clinical recognition of local peritonitis is bound the question of early operation. The majority of cases of appendicitis pass off without any need for operation, which can then be done in the quiet interval. But many lives would be saved if the more severe cases were submitted to operation as early as possible-within twenty-four to forty-eight hours of the onset.

Having thus indicated the condition and its extreme importance, we must refer again to the signs already advised for the purpose of estimating the progress of the case, viz. the pulse, the temperature, the vomiting, the abdominal signs, etc. The leucocytosis usually does not help much at this stage, but an early and marked increase of the number of white corpuscles would indicate the need for operation. Yet it is far more practicable to learn to rely on simple clinical observations than on any procedures requiring special knowledge and training.

When the enormous change in the mortality is considered all must feel most strongly that it is infinitely better to forestall the peritonitis by operation than to risk the dangers of its delay. There seems no particular reason why early, and the earlier the better, operation need have any higher mortality than ordinary appendicectomy in a quiet interval. 


\section{SURGERY OF THE APPENDIX}

V. THE DIFFERENTIAL DIAGNOSIS OF APPENDICITIS WITH DIFFUSE PERITONITIS.

One can now enter into the differential diagnosis of the four great classes of acute abdominal disease, appendicitis, intestinal obstruction, intussusception, and perforation, beginning with that of greatest frequency after appendicitis, intestinal obstruction other than that due to intussusception. It has been thought better to separate the former from the latter owing to the great differences of age, signs, etc. The relative frequency and importance of these classes is already given, and consequently they offer the most natural, simple and practical way of attacking such a difficult question as that under discussion.

(a) Intestinal Obstruction.--Obstruction is most common at an age, the decade fifty to sixty, much after that at which acute appendicitis is, ten to thirty. But the diseases certainly concur with sufficient frequency to render the discussion of the diagnosis most imperative. The mistake is a long way more frequently made in regarding appendicitis cases as obstruction rather than vice versâ. There is an excellent foundation for such opinion; for the former cases are definitely suffering from obstruction due to the paralysis of segments of the bowel as well as from inflammation and septic intoxication. Whilst the latter have, besides the obstruction, septic absorption from within the lumen of the bowel. It will be seen, therefore, that the two classes differ in the presence of inflammation and toxic paralysis of the bowel in those due to appendicitis. But cases of obstruction frequently get peritonitis. Hence both may be and frequently are cases of peritonitis, intestinal obstruction, and septic intoxication. And it will be obvious that in the later stages a differential diagnosis from the signs and symptoms will be impossible. In the earlier stages differentiation can be made. 


\section{THE "ACUTE ABDOMEN"}

At the onset the symptoms are the same, viz. those of peritonism, as has been already explained, and no diagnosis as to its origin can be made. As the peritoneal shock passes off, the case enters on the phase of its course when the origin of the trouble can, in far the majority of cases, be made out with reasonable certainty, though this may require more than one examination. In the onset the occurrence of a rigor is the only point which will aid in the formation of an opinion between these two classes of cases. A rigor is exceedingly rare in intestinal obstruction, whilst it is not uncommon in severe appendicitis. The temperature is practically invariably raised in the latter, and is subnormal in the former. The rate and character of the pulse beats is not of so much help. In both the respirations tend to be thoracic, and this tendency is nearly absolute in appendicitis, yet, whilst in obstruction the belly may be observed to move at times if not always; in inflammation the patient can rarely be persuaded to take a deep breath. The pain is of little value, though stress has been laid on the fact that in obstruction pressure may relieve the pain, whilst in peritonitis it increases it. This can be observed sometimes, but the pain elicited by percussion is more reliable than that by palpation. Percussion, even when it is quite light, in appendicitis and peritonitis very often gives rise to far greater pain than palpation. In obstruction it is always painless, whilst palpation is far more frequently painful. Vomiting and constipation are more than likely to mislead rather than help one in forming an opinion. The state of the abdomen is most valuable. Typically, in peritonitis this is immobile and rigid, in obstruction it is mobile and soft. All degrees between these extremes are seen clinically. In both, the abdomen becomes distended, and vermicular movements may be seen in obstruction and not in peritonitis. This is not always true in the less acute forms of peritonitis which may be associated with inflammation of the appendix. Also 


\section{SURGERY OF THE APPENDIX}

in the severcly toxic or the last stages of peritonitis the abdominal wall previously rigid becomes soft and pliable again, In appendicitis the patient lies quiet, with flexed hips and knees, the arms often being held above the head. In obstruction there is often extreme restlessness intensified by the griping pains.

The forms of obstruction most likely to be confused with appendicitis are those due to bands, adhesions, and Meckel's diverticulum.

(b) Intussusceptions.-Intussusceptions form the largest group of the remaining classes of the "acute abdomen." Excepting strangulated hernia, intussusceptions form the largest division of the factors of acute obstruction, viz., 35 per cent. against 30 per cent. of tumours of the bowel which comes next. With regard to age-

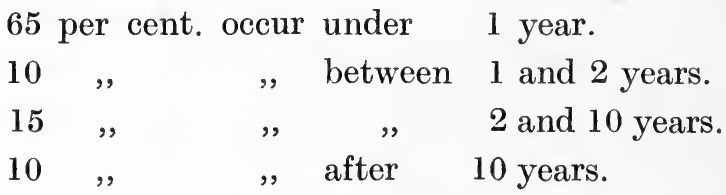

Ninety per cent. occur under ten years of age, that is, before the time when acute appendicitis is at all common. The differential diagnosis is not usuálly a matter of any difficulty. In one there is the abdominal tumour, the tenesmus, the passage of blood and mucus per rectum, the mobile and more or less pliable abdomen, etc., contrasting very distinctly with the picture of appendicitis with general peritonitis already given. A rectal examination may prove the existence of the intussusceptum in the rectum.

Perforations.-The differential diagnosis from perforations of other parts of the alimentary canal is very difficult. Of these perforations the most common is that of the stomach, next that of the duodenum. The former are chiefly found in women, especially between the ages of twenty and thirty ; the 


\section{THE "ACUTE ABDOMEN"}

latter in men between the ages of thirty and fifty. Appendicitis with diffused peritonitis is most common in male between the ages of ten and thirty. As to relative frequency, the appendix cases are eight to nine times more frequent than the gastric, fourteen times more frequent than the duodenal, and three to four times more frequent than all the perforations of the alimentary tract. In doubtful cases, therefore, there is a greater probability that the appendix is the source of the symptoms.

The cases are frequently very misleading, as owing to the presence of gastric contents in the pelvis, the peritonitis there may mask the symptoms in the upper half of the abdomen. The chief points in favour of gastric ulcer will be a history of dyspepsia, hæmatemesis, etc., the onset of acute epigastric pain, and vomiting, with shock and collapse followed by the appearance of the signs of peritonitis and free gas in the abdomen. With regard to the latter point, which is never seen in perforation of the appendix, the disappearance of the liver dullness is only of value in conjunction with a nondistended or retracted abdomen, this combined with shifting resonance over the liver or in the flank, bell note on percussion and so forth, are the chief indications. The perforation of a duodenal ulcer gives rise to similar symptoms, but in the history the pain comes on an hour or so after the taking of food; there may have been both hæmatemesis and melæna, or only the latter. Vomiting has been stated to be frequently absent in these cases, but this is doubtful.

The above points are the chief ones which will assist in distinguishing the cases. And yet again let the warning be given, "Do not allow yourself to be led away by the signs of pelvic inflammation associated with perforations in the upper half of the abdomen." Even at the operation the superficially inflamed appendix or tubes have been supposed to be the fontes et origines mali in such a case and have been 


\section{SURGERY OF THE APPENDIX}

removed. the true condition being only found at the necropsy.

Pelvic Diseases.-In this connexion it may be stated that 6 per cent. of "acute bellies" arose from disease of the female pelvic viscera. It is rare for an acute peritonitis to arise from salpingitis, and nearly half of the gynæcological cases were of ruptured tubal pregnancies. It is only about these that remarks will be made, and it is a deserving subject, as such cases frequently escape the gynæcologist's diagnosis owing to the absence in them of pelvic physical signs. In these cases there is the history of an irregularity of menstruation, typically that a period has been missed a few days, there is a sudden onset of pain in the lower part of the abdomen accompanied by vomiting, and often a little bloody discharge per vaginam. Later, the abdomen becomes distended and tender, the vomiting may increase, the bowels are constipated, the abdominal walls are almost immobile and a little rigid. The patient becomes blanched in a degree according to the amount of the hæmorrhage. Yet, on examination, little or nothing can be learned per vaginam; usually the flanks are resonant, and any dullness found does not shift as fluid does. Later still the restlessness, subnormal temperature, soft and extremely rapid pulse, etc. of internal hæmorrhage will become more and more manifest. When the abdomen is opened, the surgeon is surprised at the large amount of blood and clot present; the paucity of physical signs not having led him to expect such a quantity.

The differential diagnosis has now been discussed for five groups of cases, which together form 93 per cent. of all acute abdominal cases. The remaining seven consist of various anomalous diseases, which it would be useless to detail when it is desired that the subject should be left clear and not obscured in any way. 


\section{THE "ACUTE ABDOMEN"}

VI. THE ACUTE ABDOMEN WHEN THE DIAGNOSIS OF ITS ORIGIN

IS IMPOSSIBLE.

It has been pointed out that in the stage of onset of appendicitis it is impossible to diagnose it from any other acute abdominal mischief. As the disease develops it becomes recognizable and remains so until the consequent peritonitis becomes so diffuse as to mask all traces of its source. In these cases, an opinion based on probability can be formed by means of consideration of the history of the case, as excluding gastric ulcer, obstruction following operations by adhæsions or bands, typhoid fever, tubal gestation, and so forth. If the age of the patient is under one year it would suggest intussusception, ten to thirty years appendicitis, twenty to forty-five gastric and duodenal ulcers, forty and onwards obstruction. The male sex are more liable by far between the ages of ten and thirty to the acutest form of appendicitis, in the ratio of five to one female. The latter between fifteen and thirty may be cases of perforated gastric ulcers, but males with perforated duodenal ulcers are usually older than this. Finally, let it be borne in mind that the appendix is responsible for far and away the majority of cases of acute abdominal disease in the young.

Our powers of diagnosis are wanting in differentiating the cases that we may save from those beyond hope. And with a mortality of 84 per cent. against us, abdomina are opened in the hope that the case may be one of the chosen sixteen. When the abdomen is open the smell of the pus usually and definitely indicates its origin. Pus from perforated gastric and duodenal ulcers is very rarely offensive. The next perforation in order of frequency which was found at St. Thomas' Hospital was that of the rectum. Operation usually settles all doubts, but let it be remembered that the appendix has been wrongly regarded as the offender and removed for almost every other kind of acute abdominal disease. 


\section{CHAPTER IX}

Intussusception-Some of the Rarer Diseases of the Appendix-Benign Tumours-Sarcoma-CarcinomaActinomycosis-Tuberculosis

\section{INTUSSUSCEPTION OF THE APPENDIX}

$\mathrm{Br}$ intussusception of the appendix is generally understood its inversion into the cæcum; which event is followed by the invagination of that structure into the ascending colon. Such a condition is undoubtedly rare, but as the majority of the cases have been recorded during the last few years, it may be safely prophesied that they are by no means so uncommon as has hitherto been supposed. A careful examination of the literature has led to the discovery of the reports of seventeen cases. In all of these the inversion was complete, and there was no scrap of evidence to show its pathogenesis. A paper then appeared by Dr. Rolleston, in which he described a case of prolapse of the mucous membrane of the appendix into the cæcum. It was found at the post-mortem examination of the body of a woman aged thirty-two, who had died of peritonitis secondary to the perforation of a duodenal ulcer. The prolapse was obviously caused by the peristaltic action of the appendix excited by the presence of a concretion.

The true significance of this case has not been realized. It is the only adequately recorded case of partial inversion of the appendix into the cæcum, and gives the key to the explanation of those cases of total inversion associated with invagination of the caput coli. Up to this date the subject had merely been one of speculation. 


\section{INTUSSUSCEPTION OF APPENDIX}

The small size of the lumen and the relatively great thickness of its walls prevent the invagination of the appendix into itself. Arguing from Rolleston's case, the invagination may be secondary to the presence of a concretion, a mass of secretion, a swollen lymphoid follicle, or the like, which is

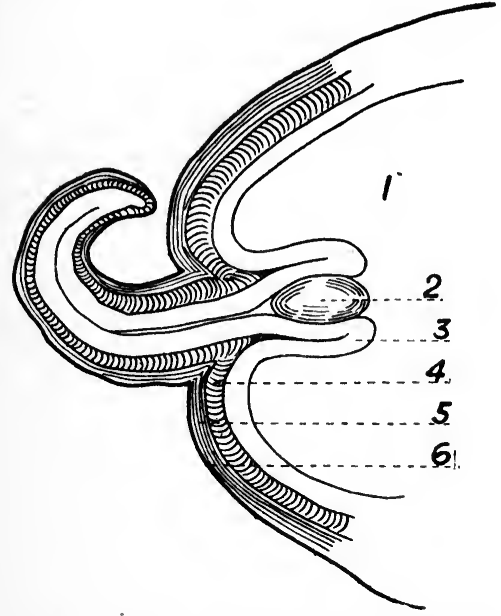

Fig. 10. Cæcum. Heavy line represents the peritoneum; the fine line mucous membrane. 2. Fœcal concretion, covered by mucous membrane, projecting into the cæcum. 3. 4. 5. 6. Layers composing the cæcal wall. protruded into the cæcum by the muscular action of the appendix behind it. The appendix forms the intussusceptum and the cæcum the intussuscipiens. This variety differs from all the others, in that owing to its large size, the cæcum (the intussuscipiens) cannot contract closely enough to grasp, and still further invaginate by traction, the appendix (or intussusceptum). The inversion is very largely due to the vis a tergo on the part of the appendix and vis a fronte being largely lacking. In such a way as this the appendix may be turned inside out. Otherwise it is difficult to see how mere prolapse of the mucous membrane can progress further owing to the inability of the cæcum to grasp it and pull. Viewed in this light, Dr. Rolleston's paper is a most valuable contribution to the pathology of the cases under discussion.

The condition produced by the inverted appendix may be likened to that produced by a long uvula in the pharynx. Both cæcum and pharynx are excited to muscular contractions, which in the case of the former cause the caput 
coli to be drawn up into the cæcum. If it is drawn up sufficiently far to obstruct the ileocæcal valve for an appreciable time, an attack of abdominal pain is experienced by the patient. But unless the caput coli is well home above the valve, the cæcum seems unable to seize and drag it onward, and the colic passes away, the valve becoming unblocked again owing to the recession of the caput coli and the appendix. As these attacks succeed each other the recession becomes less and less perfect, due to the chronic inflammatory changes and the formation of adhæsions, until at last the ascending colon grasps the intussusceptum, and superimposes an acute or subacute colic intussusception with the aid of the vis a tergo of the small intestines. Clinically these cases are very chronic and at operation or post mortem it is generally impossible to reduce the intussuscepted mass.

These are examples of double intussusceptions associated with the appendix. The primary invagination consists of the appendix followed by that of the caput coli. Later a secondary colic form is superimposed on account of the primary invagination blocking the ileocæcal valve. Seventeen cases have been recorded, all chronic, giving a history of a long period during which the patient was subject to colicky pains, vomiting, etc. Twelve occurred in males, four in females; sixteen occurred in children between two and a half and nine years; one occurred in a woman of forty-two. Eleven recovered and six died. In seven the gut was resected, of which five recovered; in eight it was reduced, with six recoveries; in three the condition was found post mortem.

It is extremely interesting to note that in no case had the inversion caused the appendix to slough. In the inverted position the blood stream is not of necessity stopped; and it may not be even seriously interfered with. And again, the inverted position so alters things that the appendix no longer offers a culture tubelike recess for the nurture of organisms. Dr. Wallace, whose 


\section{INTUSSUSCEPTION OF APPENDIX}

original paper it was not possible to consult, records a case of the passage of an appendix in a stool, but whether this was inverted or merely from an appendix abscess, is not known.

One further remark may be made. All except one of these cases occurred at an age before that at which appendicitis is common, and in no case was there any history of earlier inflammations of that organ. In point of fact, appendicitis will very likely leave behind it such fibrosis, stricture, adhæsions, etc., that it may be regarded as protective against inversion. The chronic appendicitis that leads to the formation of concretions may be rarely an exception to this. Owing to the inversion of the process, concretions would be turned into the colon and consequently be unnoticed.

\section{LITERATURE}

\section{Partial Inversion of the Appendix}

Rolleston, Edinburgh Med. Journai, 1898, n.s. iv. 21-26.

D'Arcy Power, Case mentioned in a discussion, British Medical Journal, 1897.

Complete Inversion of the Appendix, with the subsequent formation of a Second and Colic Intussusception.

McKiidd, Edinburgh Medical Journal, 1858.

Chaffey, Lancet, 1888.

Renshaw, and Wright, British Medical Journal, 1897.

McGraw, British Medical Journal, 1897, ii.

Pitts, Lancet, 1897, i. 1602.

Waterhouse, Path. Trans. London, xlix, p. 108. Lancet, 1897, ii

Westermann, Wieblad van Niederlander Tydschrift von Geneskunde; No. 24.

Lees, Lancet, 1898, i.

Enderlen, Münch. Med. Woch., 1900, July, 1021.

Haasler, Arch. fur Klinische Chirurgie, Bd. 68, Hft, 3, Fall. 9; Ibid. Fall. 3.

Montsarrat, Liverpool Medico-Chirurgical Journal, 1901, xxi. 68-78. Ackermann, Beiträge zur Klinische Chirurgie, 1903, Jan., 579.

Haldane, Scottish Medical and Surgical Journal, 1903, xii. 4, p. 333.

Bishop, Chironian, New York, 1902-3, xix. 81-84.

Hogarth, British Medical Journal, 1903, April, i. p. 850.

O'Connor, Lancet, 1903, Vol. ii. p. 600.

Corner, Annals of Surgery, 1903, Nov., p. 708.

Passage of Appendix per Rectum.

Wallace, American Medicine, 1901. 


\section{SURGERY OF THE APPENDIX}

\section{GENERAL INTRODUCTION TO THE RARER DISEASES OF THE APPENDIX.}

The diseases of the appendix other than appendicitis are all said to be rare or uncommon. As there are several common factors which have given rise to this opinion, it will be as well to point these out. Any disease of the appendix, such as syphilis, tubercle, sarcoma, carcinoma, etc., leads to some obstruction to the normal expression of its contents. This may be the result of a mechanical obstruction, or the presence of the disease may render the peristalsis incompetent. or most probably there is a combination of both these factors. The result is that the secretion is retained and organisms grow luxuriantly. A septic attack of appendicitis is very prone to arise early in the disease, and the case may be diagnosed, treated, and perhaps the appendix removed before any characteristic macroscopical changes have become sufficiently obvious to attract attention and cause a microscopical examination to be made. In this way it seems probable that the true fons et origo mali is over-looked, being masked by the secondary septic process. So far, the majority of cases of carcinoma, tubercular disease, etc. of the appendix have been described within the last few years, and it may be confidently expected that further systematic research will reveal the fact that they are far more common in reality than has been suspected. It will thus be seen that both clinically and pathologically these cases seem to be merely appendicitis.

\section{BENIGN TUMOURS}

Benign tumours of the appendix are very rare. Lafforgue ${ }^{1}$ in 1883 could only find references to six, one being a lipoma, one a myoma, two lymphadenomata, and two hydatids. Since then there have been a few more cases reported. Kan-

1 Lafforgue, Thèse de Lyon, 1883. Quoted in Bull. de la Soc. Anat. Paris, 1897, lxxii. 814. 


\section{SARCOMA OF THE APPENDIX}

thack and Lockwood (Brit. Med. Journal, 1895, i. 756) gave an account of a psammoma. Deaver (Appendicitis, 1901, p. 95-6) reports two cases in which two and one fibromyoma respectively were found in the appendix, both cases were operated on for fibromyomata of the uterus. The only other record, besides, is of a granuloma that formed round a septic silk ligature in a patient under the care of Prof. Routier. All were found whilst exploring, ante and post mortem, for other conditions. It is most rare for them to give rise to any clinical symptoms. In point of fact they are chiefly of pathological interest.

Birch-Hirschfeld ${ }^{1}$ has reported a case of hydatid cyst of the appendix, and Scholler ${ }^{2}$ one with hydatids of the liver also. Wood, in the University Medical Magazine for 1899, has given an account of cystic distension of the appendix.

\section{SARCOMA}

Sarcoma of the appendix seems far more uncommon than carcinoma. In 1893 Gilford recorded a case which occurred in a man of twenty-seven who had had chronic inflammation about his appendix for years. A mass in the right iliac fossa was excised and reported on as sarcomatous. As there was no report by a special pathologist it may be as Dr. Rolleston has suggested that the tissue was in reality the result of chronic inflammation. In 1895, Dr. Glazebrook recorded a case of sarcoma of endothelial origin, but unfortunately his paper could not be consulted, and there is no satisfactory quotation. In 1897, Dr. Collins Warren excised a tumour with the cæcum, appendix, and contiguous ileum. The tumour from the description may not have arisen in the appendix. In the same year, Mr. Murray published a case of a boy of six years of age,

1 Quoted by Heller, Invasions Krankheiten von Ziemssen's spec. Path. und Therapie, 1876, iii. 331.

2 Quoted by Bierhoff, Deutshe Arch. fur Klin. Med., 1880, xxvii. 248. 


\section{SURGERY OF THE APPENDIX}

who had a tumour in the right iliac fossa, and died eight months later from apparent diffusion of the growth. At a subsequent examination of the sections, Vissmann cast doubts upon the sarcomatous nature of the tumour. In 1900, Davis recorded the first authentic case of sarcoma of the appendix, which he removed successfully from a man of fifty-one. At the end of five months there was no recurrence. In 1903, Patterson added another bona fide case, occurring in a man aged thirtynine. This man gave a history of recurrent attacks of appendicitis each lasting three or more days. There was a mass in the iliac fossa and a tumour to be felt. Death occurred shortly after the operation, at which the appendix was found to be bent at a right angle and was much swollen beyond the bend. Microscopically it proved to be a round celled sarcoma.

All these cases were diagnosed as appendicitis, and as in tuberculous appendicitis, etc. the septic factor attracted the attention clinically and pathologically, so masking the true changes. It is this complicating septic process which occurs whilst the disease is yet young and renders the case fatal or demands the ablation of the appendix before the tumour is macroscopically recognizable. Hence the apparent rarity of such cases.

All these six cases occurred in males, the two authentic cases at the ages of thirty-nine and fifty-one. The youngest reported case was in a boy of six.

Gilford, Lancet, 1893, ii. 241.

Glazebrook, Virginia Semimonthly, May, 1895.

Collins Warren, Boston Medical and Surgical Journal, 1897, Ixxxviii. 177.

Murray, Medical Neus, New York, 1897, 1xx. 543.

Davis, Journ. Amer. Med. Assoc., 1900, xxxv. 1556.

Patterson, Practitioner, London, 1903, lxx. April, p. 515.

Corner and Fairbank, Path. Soc. Trans., London, 1904.

\section{ENDOTHELIOMA}

Pathologists are not decided or unanimous upon the position and pathology of the class of tumours called endotheliomata. 


\section{CARCINOMA OF THE APPENDIX}

And considering the difficulties that surround their identification this is not surprising. One case of endothelioma of the appendix has been recorded by Deaver (Appendicitis, 1901, p. 100), and which occurred in a series of 706 examinations of appendices. It was found in a woman, twenty-four years of age, who gave a history of four previous attacks of appendicitis. The case, as is always so with the uncommon diseases of the appendix, was diagnosed as merely inflammatory. The organ was removed and carefully examined. A most thorough microscopical description is given which seems to make the diagnosis of endothelioma certain. Owing to the presence of organisms in the lumen of the appendix every pathological change is accompanied by inflammation which may mask the nature of the case anatomically, and certainly clinically.

\section{CARCINOMA}

Primary carcinoma of the appendix is an uncommon disease. Maydl, Nothnagel, Leichtenstern, Müller, Bryant, Zemann, and Deaver, have all published statistics of carcinomata of the bowel, which when added together form a total of 2,262 cases, nine of which arose in the appendix, i.e. 1 in 251. Careful inquiries into the literature have revealed the records of 40 cases, well over half of which have been published within the last five years. The united statistics of the above authors may be taken as putting the frequency of carcinoma of the appendix too low, because it has now been fully proved that unless microscopical examinations of the appendix are systematically made these cases are overlooked. And again, the malignant growth is always accompanied by inflammatory changes which may mask the true origin of the disease both pathologically and clinically. The cases always come under notice as appendicitis, or else the condition is discovered by chance during an operation or as a result of a careful post- 


\section{SURGERY OF THE APPENDIX}

mortem examination. It may be expected confidently that this disease will be found to be a great deal more common than is supposed. Letulle and Weinberg whilst examining cases of so-called obliterative appendicitis found two cases of carcinoma amongst them.

The carcinoma is most frequently found in the terminal part of the appendix, and it is in this situation that the organisms are prone to convert the case into one of acute inflammation with abscess or peritonitis. The growth may also begin in the proximal part and secondarily involve the cæcum. From the cases reported, 48 per cent. began at the distal extremity, 15 per cent. near the cæcum, and 37 per cent. between. Those situated near the cæcum may have arisen there and secondarily involved the appendix. Whipham's, one of Kelly's, and Wright's cases certainly originated in the latter; Beger's probably started at the valve of Gerlach; those recorded by Bishop, Monks, Kolaczek, and Regling were of cæcal origin. Besides being secondarily involved from the caecum the appendix may become carcinomatous secondarily to the ovary as in the instance reported by Hodder.

It is not out of place to say a few words about carcinomata of the large bowel. The tendency of these tumours is to spread round the bowel rather than along it, so forming the ring carcinomata. This line of infection is along the course of the blood-vessels and lymphatics. In the caput coli these no longer run circularly, but obliquely, so that in this situation there is more tendency for the carcinoma to spread from segment to segment than there is in any other part of the large bowel except the rectum. The connection with the ovarian lymphatics, viâ the appendiculo-ovarian ligament, explains the involvement of the appendix from a carcinoma of the ovary (Hodder) ; and that of the ovary from a carcinoma of the appendix (Whipham).

The disease is more frequent in females than males in the 


\section{CARCINOMA OF THE APPENDIX}

proportion of eight to five. Seventy per cent. gave a history of a previous attack of appendicitis. But the most striking feature about these cases is the early age at which carcinoma is recognized. The youngest was a girl of 12 , one at 15, two at 19 , one at 20 and one at 23 , three at 24 , one at 25,26 , and 27 respectively, two at 30 . Almost all of the younger subjects were women, and the older, men. Carcinoma is found at the age at which appendicitis is most common, and doubtless there is a causal relationship between the two.

It must be remembered that even a small tumour may obstruct the lumen of the appendix. The result of this is that secretions and organisms are dammed up behind, and the case takes upon itself all the characters of ordinary appendicitis. In this way its true origin is masked. And though carcinoma is undoubtedly rare as compared with the extreme frequency of appendicitis it may safely be looked upon as being a great deal more common than is thought. The addition of this inflammatory process explains the fact that carcinoma of the appendix is said only rarely to give rise to secondary deposits, the patient dying, the growth sloughing, or the appendix being removed before any metastasis occurs.

Lejars' case exhibited a rapid return of the carcinoma and dissemination of the growth, although at the operation the appendix externally appeared almost normal.

LITERATURE.

* Merling, Journal d'Experience, 1838.

Rokitansky, Mediciniscshe Jahrbuch, 1867, xiii. p. 179.

* Prus, Thèse de Croizet, 1865.

* Lafforgue, Thèse de Lyon, 1883.

Beger, Berliner Klin. Wach., 1883, p. 616.

Draper, Boston Medical and Surgical Journal, 1884.

Wright, Ibid, 1898, cxxxviii. p. 150.

Stimson, Annals of Surgery, 1896, xxiii. p. 186.

Elting, Ibid., 1903, April, p. 549.

Moskowitz, Ibid., 1903, June, p. 891.

Cullen, John Hopkins Bulletin, 1899.

Hodder, Ibid., 1900, p. 175.

Kelly, Deaver's Appendicitis, 1901. Trans. Path. Soc.. Philadelphia, 1901. 


\section{SURGERY OF THE APPENDIX}

* Zemans, Bibliothek der Med. W'issen. Vom Drasche, iii. 49. Goffe, Med. Record, New York, 1901, lx. p. 14.

McBurney, Ibid., 1901, lx. p. 478.

Lartigan, Quoted by McBurney.

Jessup, Med. Record, New York, 1902, 1xii. p. 289.

Weir, Ibid.. 1903, May, p. 802.

* Girard, S. Cyprien, Toulouse, 1900.

Whipham, Lancet, 1901, i. 319.

Rolleston, Lancet, 1900, ii. 11.

$\dagger$ Panchet, Gaz. Méd. de Picardie, Amiens, 1900, xviii. 146.

Ruyter, Arch. für Klin. Chirurgie, lxix. 281.

Regling, Deutsche Zeitschrift für Chir., lxv. 376.

Letulle and Weinberg, Bull. de la Soc. Anat., Paris, 1897, 1xxii. 747.

Mossé and Daunic, Ibid., 1897, 1xxii, 814.

Lejars, Bull. and Mém. Soc. Chir., Paris, 1897, xxviii. 96.

Fairbairn, S. Thomas' Hosp. Reports, 1901. Case of Dr. Cullingworth's.

Harte and Willson, Med. News, New York, 1902, lxxxi. 193.

* Quoted by others. † Not consulted.

\section{ACTINOMYCOSIS}

It is often a very difficult question to decide as to whether the disease is primary or secondary in the appendix. As this organ is so situated that it represents the backwater and sample culture tube for the cæcum, which is the firstresting-place of the products of digestion after leaving the stomach, it would be surprising if actinomycosis was not found there. In any intestinal form, and especially that of the appendix, the streptothrix actinomycosis will be accompanied by other organisms which, owing to their action, are very likely to render it difficult or even impossible to demonstrate the true origin of the infection. Clinically the case runs a chronic course exhibiting four stages, the first with irregular abdominal symptoms; the second with the presence of a tumour; the third with fistulæ; the fourth during the process of repair. But it seems most probable that owing to the mixed character of the infection some of these cases are more acute than others, and then the presence of the streptothrix may be of quite secondary importance.

The frequency with which the appendix and the ileocæcal region is affected has been carefully studied by some authors, the result of whose figures shows that, of cases of abdominal 


\section{ACTINOMYCOSIS OF THE APPENDIX}

actinomycosis, this region is affected in 50 to 60 per cent. of the examples. In all, about 50 cases have been reported.

The diagnosis is only made by the discovery of the streptothrix which may be most difficult to find, whilst the mycelium itself is still less easy. The treatment of these forms is in no way different from that of actinomycosis elsewhere. They differ from appendicular abscess in chronicity, the size of the mass in the right iliac fossa, and the persistence of the sinuses. The history is frequently most misleading, and owing to the very mixed character of the infection the reaction to iodide of potassium is slow. It will thus be seen that these cases present, in many instances, the greatest difficulty in diagnosis. Abdominal actinomycosis if not treated early by potassium iodide or by operation, is frequently fatal in its result from exhaustion due to the chronic sepsis, fæcal fistulæ, and discharge. In some cases, as in the first reported by Mr. G. H. Makins, secondary deposits of actinomycosis may occur in the liver, and more rarely the dissemination may reach other organs.

\section{Bibliography.}

Böstrom, Ziegler's Bieträge. Allgemeine Path. und Path. Anat., ix. i. 1891.

Gangolphe and Duplant, Revue de Chirurgie, xvii. 503, 1897.

Grill, Beitrage zur Klin. Chir., xiii. 551, 1895.

Guder, Rev. Méd. de la Suisse Romande, 1891, xi. 746.

Hinglais, Thèse de Lyon, 1897.

Kern, Correspondenzblatt, Schweitz. xxi. 570, 1891.

Lange, Annals of Surgery, xxiv. 371, 1896.

Lanz, Correspondenzblatt f. Schweitz., xxii. 307, 339, 1892.

Latimer and Welch, Trans. Assoc. of Amer. Physicians, 328, 1896.

Ransom, Medico-Chir. Trans., lxxv. 85, 1892.

Ruhräh, Annals of Surgery, xxx. 1899.

Sonnenberg, Path. und Therap. du Perityphlitis, Leipzig, 1897.

Eccles, Lancet, i. 1903.

Thèvenot, Gaz. des Hôpitaux, 1417, 1902.

Daske, Inaug. Dissertation, Greifswald, 1902.

Hofmeister, Verhandl. d. Gesell. Duetsch. Naturf. und Aerzte, 1899, 1900, 2 Th., 2 Hälfte, 113, 115.

Bäärheim, Hygeia, 1900, lxii. 545-50.

Ekehorn, Upsala Lakarsaf. F'örh., 1900, n.f.v. 455-9.

Makins, St. Thomas' Hosp. Reports, 1896, xxv. p. 135. 


\section{SURGERY OF THE APPENDIX}

The above contain references to cases by Barth, Braun, Bergmann, Illich, Isräel, Langháns, Lührs, Körte, Pontick, Regnier, Roux, Samter, Schreyer, Uzkow, Vassilier, Murphy and Lee, Ullman, Ozerung and Haddaeus.

\section{TUBERCULOSIS}

Primary tuberculosis of the appendix seems to be an uncommon disease. Even secondarily it does not seem to be frequent. Drs. S. Fenwick and Dodwell found it diseased in 17 out of 2,000 fatal cases of phthisis. On the other hand, it is not so uncommon for surgeons to find tubercular disease of the glands in the base of the mesoappendix and at the ileocæcal angle. Bouglé, ${ }^{1}$ in a recent paper, says that if careful and thorough examinations are made of appendices, primary tubercular disease will be found to be far more common than is supposed. He emphasises the fact that it may be found in a sclerous, caseous or suppurating form, and from his account the base of the appendix rather than the tip is affected, and he advises resection of the cæcum. No example of a cretified tubercle in the appendix has been recorded though it is comparatively common to find cretified glands near it. As in other cases the presence of organisms in the appendix may by their action mask the original tuberculous origin of the disease. There can be no doubt whatever that the secondary septic infection frequently obscures the true diagnosis.

The chronicity of many of the cases is also very marked, and an instance may be quoted which exemplifies this, and also a class of case in which a tubercular origin may be suspected. A. B., a man aged about thirty-five, a year previously had an abscess low down in the iliac fossa opened by his doctor. A sinus resulted, also a fæcal fistula and a hernia through the scar. The fistula would not close, so an operation was recommended to bring about that end. The fistula was excised, and the appendix and cæcum withdrawn from the abdo1 Arch. Gen. de Méd., Feb. 3, 1903, Paris. 


\section{TUBERCULOSIS OF THE APPENDIX}

men. The former was thickened, inflamed and coiled up under the latter. At its base was a good sized perforation which was adherent to the scar and caused a fæcal fistula. The appendix and the neighbouring part of the cæcum were excised and the wound sewn up. It healed by first intention. But the man died two years later of acute pneumonic phthisis. Microscopically the appendix was tubercular.

The infection is rare through the blood except in cases of acute miliary tuberculosis. Most examples occur at the proximal end and involve also the contiguous part of the caput coli which is not always examined. Hence it is close to the cæcum that the perforation of tubercular ulcers is most likely to occur. And it may be laid down that lesions in this situation should always lead to the suspicion of a possibly tuberculous origin, especially so in chronic cases, although the last attack may be merely a septic exacerbation. Hence it may be pointed out that septic appendicitis chiefly affects the distal, whilst tubercle affects the proximal portion.

Mr. Mayo Robson (Lancet, 1902, ii. p. 851) records such a case amongst other examples of abdominal tuberculosis, and suggests that in cases of recurrence of tubercular peritonitis after the operation of incision and lavage, the result may be due to the fact that a focus of disease has been left behind. It may be pointed out that in far the majority of cases the abdomen is not completely explored, and that plenty of disease in the peritoneum and perhaps in the viscera, is left behind in every case. The recurrence can hardly be attributed to the fact that a small focus has been overlooked, such as in the Fallopian tube, ovary, or appendix. Many cases have recovered where no attempt whatever to discover a focus had been made.

Fitz has given the frequence of tubercular disease as eight in 257 diseased appendices, or about three per cent. Fenwick and Dodwell ${ }^{1}$ found the ileocæcal region affected in 85.5 per

1 Fenwick and Dodwell, Lancet, 1892, ii. p. 133. 


\section{SURGERY OF THE APPENDIX}

cent. of cases where the alimentary canal was involved, and as these formed $56^{\circ} 6$ per cent. of the whole, this region would be affected in about 49 or 50 per cent. of those cases which they examined, that is to say fatal cases of tuberculosis.

These statistics are not of great value in estimating the probable frequency of the disease, and it remains for future investigations to give us more correct ideas. 


\section{SUBDIAPHRAGMATIC ABSCESS}

\section{CHAPTER X}

Complications of Appendicitis (Contd.): SubdiaPhragmatic and other Secondary AbscessesEmpyema and Lung Diseases-Cardiac and Renal Affections-Thrombosis of Veins-Hhamorrhages - Chronic Cellulitis of the Iliac FossaMrositis of the Illiacus and Psoas Muscles

\section{SUBDIAPHRAGMATIC AND OTHER ABSCESSES}

These are briefly classified into those due to direct extension of infection and those in which it is transferred by the blood or lymphatic streams. Those due to direct extension of the appendicitis will be considered first, and may be subdivided into two classes :-intra and extra-peritoneal.

The appendix is almost invariably a structure completely invested by peritoneum, except of course at the point where it is attached to the cæcum. And it may be said that almost every appendix abscess is intra-peritoneal. The abscesses are sometimes so completely enclosed by adhesions that they gave former observers the idea that they were extra-peritoneal. This is not so. The abscess is often situated internal to the cæcum, very frequently dipping down into the pelvis. It may also be iliac, i.e. in the iliac fossa, or lumbar, in the loin. Infection may spread up the outer side of the colon and form a subhepatic or a subdiaphragmatic abscess, limited on the left by the falciform ligament of the liver. Or it may pass in front of the ileum along the inner side of the ascending colon to the fossæ in front of the right kidney, that is, the prerenal or 


\section{SURGERY OF THE APPENDIX}

subhepatic fossa. The same region may be reached by crossing from the outer to the inner side of the ascending colon. Or, it may spread to the pelvis, forming an abscess in the prerectal pouch, or further afield along the rectum, sigmoid flexure, or pelvic colon, to the left iliac fossa, left loin, under the spleen, or between that organ and the diaphragm.

The lesions situated far from the primary focus have given rise to the idea of the possibility of a "distant" peritonitis, or that a peritonitis previously general has quieted down except for an abscess or two. But the explanation for these conditions is best understood by direct extension of the infection up either side of the ascending colon, or viâ the pelvis up either side of the descending colon. All these varieties of abscesses have been described. Drs. C. R. Box and A. E. Russell give an excellent description of some of them in a paper in the St. Thomas' Hospital Reports, 1897, xxvii. p. 105.

The recognition of the existence of a subdiaphragmatic abscess is a matter of great importance as it is a not infrequent complication of both appendix abscess and peritonitis.

The diagnosis of them clinically is a matter of considerable difficulty. Usually the appendix abscess has been opened, and the temperature has fallen for a few days, and then rises again. The most common place for an appendix abscess to be insufficiently drained is in the pelvis, and the rise of temperature may be due to this cause. A rectal examination and a short period of watching usually clears this matter up. Similarly, an abscess in the left iliac fossa or in either loin, or a residual one-i.e. due to insufficient drainage, will declare its presence on examination. But subdiaphragmatic, subhepatic, subsplenic and anterenal abscesses, and empyemata have to be carefully looked for in order to be found. With the subdiaphragmatic abscess are found local pain with embarrassed respiration and rapid pulse, and an increasing dullness on percussion, an increasing area of diminished breath sounds, diminished 
vocal resonance and fremitus at the base of the lung behind, almost always upon the right side. Subhepatic abscesses are far less common and are much harder to diagnose. When such conditions are found at the base of the lung, watch the extension and progress of the signs, the pulse, temperature, and general condition. It may be a matter of uncertainty whether or not the signs are due to pleurisy, i.e. whether the trouble is above or below the diaphragm, and if signs both local and general do not progress, wait for the development of events. In a number of cases these untoward symptoms quiet down sometimes suddenly, sometimes slowly. If they progress, the advisability of exploring with a needle must be considered, but this method is very unsatisfactory as well as dangerous. The subhepatic region cannot be efficiently explored by this means. If the signs at the base behind are marked, the temperature continues to rise, and the pulse and general condition get worse, then operation must be undertaken. A rib is resected freely, at least four inches; no rib above the eighth should be selected as the excursions of the diaphragm would render the operation difficult. The middle of the resected rib is about in the posterior axillary line. The pleura is now opened, and perhaps also an empyema. If the pleura is unaffected it should be sutured to the diaphragm or not, according to the condition found. It is probably safer to insert sutures. The patient may now be left for a few days, or better, the diaphragm at once incised, and the abscess sought for with the finger.

The situation of the pus may not be, however, so definitely indicated, and then it is better to use the incision suggested by Drs. C. R. Box and A. E. Russell ${ }^{1}$ with this slight modification. The lower part of the incision is made parallel with the fibres of the rectus, beginning about the junction of the eighth rib with its costal cartilage, the fibres of the rectus are separated

1 St. Thomas' Hospital Reports, 1897, xxvi. p. 105. 


\section{SURGERY OF THE APPENDIX}

and so the subhepatic, prerenal and subdiaphragmatic spaces explored as far as possible. This incision may be extended backwards along the eighth rib, which can be resected and the transpleural operation done if required.

The treatment of other abscesses is as usual by incision and drainage ; it is very useful to drain the pelvis in women through the vagina. Similarly a collection in the loin may be drained by a puncture through the loin, as well as by the ordinary incision.

Extraperitoneal abscesses are far from common, and usually arise secondarily from an intraperitoneal one. Naturally they are most common in the right iliac fossa whence they may spread to the loin, behind the colon or amongst the perirenal fat. They do not commonly extend to the pelvis. In women the infection may and does spread viâ the appendiculo-ovarian ligament to the ovary or broad ligament. Very uncommon places for an extra-peritoneal abscess to track to are the thigh through the crural canal, under Poupart's ligament, or down the right or left inguinal canal with the spermatic cord. Intraperitoneal abscesses have been described in both femoral and inguinal hernial sacs. ${ }^{1}$

The formation of an extraperitoneal abscess usually causes an inflammation of the iliacus muscle, perhaps the psoas muscle and also the lower nerves of the lumbar plexus, as evidenced by the pains which shoot along their course into the lower extremity.

The blood stream may infect the system in two ways, by means of the portal vein to the liver, by far the most common, or, by the caval veins, the heart, and arteries to the lungs and other parts. The former is portal pyæmia and the latter systemic pyæmia. In both, the abscesses are usually multiple. The caval system almost always gets affected after the portal system has infected the liver, the hepatic veins carrying the infection to the inferior vena cava and to the right auricle.

${ }^{1}$ Corner and Spurrier. St. Thomas' Hospital Reports, 1902. 


\section{THROMBOSIS OF VEINS}

Infection by the lymphatics must be common considering the septic nature of appendicitis, but it is not much recognized. Every surgeon is familiar with the enlarged succulent glands sometimes found when operating in acute cases. But these glands never seem to suppurate. Perhaps the reason is that they are so immunized by the constant absorption of products from the bowels that they easily deal with the increased infection.

Actual suppuration has been noticed by us in two cases. In one, the gland at the ileocrcal junction was removed and found necrotic in the centre; and in the second, a retroperitoneal abscess, thought to be of glandular origin, was treated subsequently to the opening of an appendix abscess.

\section{Rare Septic Complications.}

Lung complications are-pleurisy with or without effusion, empyema, pneumonia, bronchopneumonia, pyæmic infarctions or abscesses.

Of these, empyema is the most important, being sometimes associated with abscess below the diaphragm and caused by direct extension from the abdomen. At other times it arises apparently through the lymphatics. In whatever way arising, it will be found that the colon bacillus is more or less extensively spread throughout the pus. Usually the empyema forms subacutely, but at times it develops suddenly and may then be associated with a pneumothorax, unconnected with the lung, from the development of gas in the pus. It may be that this secondary complication of appendicitis, pyopneumothorax, will altogether overshadow the original disease, and unless careful heed is given to the earlier symptoms, the origin may be quite unsuspected. The subject of this complication is often very ill indeed, but will improve in a marvellous manner after the pus has been evacuated in the usual way, unless the empyema is only part of a large abdominal collection. 


\section{SURGERY OF THE APPENDIX}

Of the rarer examples, two may be cited in illustration. In the first, a man had a left empyema and a sinus from it lasted twenty years. At the post mortem, the origin was found to have been in the diseased appendix, a sinus leading from that to the pleura. In the second, a young man was admitted to St. Thomas' Hospital who on examination was found to be in a moribund condition, which was due to an empyema on the right side, the origin of which was in the appendix.

Diseases of the heart may arise during the course of an attack of appendicitis, of which we may mention endocarditis, simple or septic, myocarditis, and pericarditis, with or without effusion.

Kidney complications, which are known and recognized, are nephritis, abscesses, and pyæmic infarcts. Abscesses in the liver, the spleen, and all the other indications of pyæmia may be present.

\section{THROMBOSIS IN APPENDICITIS}

Thrombosis may occur more or less locally or at a distance. When the appendix is inflamed the veins in its mesentery may become thrombosed, and the thrombus may extend into a mesenteric vein so leading to extensive necrosis of the bowel. Happily this is rare, but is not unknown. Or, the inflammatory process may extend to the iliac veins, especially the external when the inflamed appendix lies over it. This phlebitis leads to thrombosis which usually extends into the upper part of the femoral vein. Clinically it is recognized by the tender cordlike thickening of the vein, œdema of the leg and perhaps dilation of superficial veins to allow of the formation of an anastomotic circulation. Treatment.must be for the appendicitis as well as the phlebitis and thrombosis, and that for the latter usually involves rest for six weeks or so. In far the majority of cases suppuration does not occur in the thrombus, and resolution may be looked for. It is very un- 


\section{THROMBOSIS OF VEINS}

common for the thrombus to extend into the common iliac vein and the vena cava, so leading to thrombosis of the iliac vein of the other side. But thrombosis of both iliac veins may occur in another way than by direct extension of the phlebitis and the clot. It may arise from general causes such as feeble circulation, anæmia, etc., quite apart from the appendicitis. But also, in infective conditions of all kinds one is liable to get thrombosis of veins at a distance from the local focus. Upon the causation of these it is not necessary to enter. And it is in this way that most of these complications arise, rather than from direct extension of the inflammatory process, and the comparative rareness of a suppurative phlebitis is rendered intelligible. The clots are rarely sterile and the bacillus coli communis and proteus vulgaris have been found in them.

Suppurative phlebitis may occur as a result of appendicitis, and naturally is most common in the veins of the mesoappendix. This is an extremely serious complication, for besides perhaps causing the havoc of intestinal necrosis by extension of the clot into the mesenteric vein, septic emboli may be detached and carried by the blood stream viâ the portal vein into the liver, where they give rise to secondary abscesses. The process is called portal pyæmia. Clinically it may be recognized by the onset of local pain in the right hypochrondriac region with chills and shiverings. The temperature rises suddenly, and though it comes down a little, remains high. Thrombosis of the portal vein and pylephlebitis may occur, leading to ascites, piles and hæmorrhages from the bowel, dilatation of the esophageal veins and perhaps hæmatemesis. The superficial veins of the abdomen become dilated. Unfortunately this complication is usually of a septic nature and is very fatal.

The further results of thrombosis, such as embolism of the heart, pulmonary, cerebral or other arteries must be mentioned, also infective endocarditis, pyæmia, pulmonary infarction, etc. 


\section{SURGERY OF THE APPENDIX}

An attempt is usually made by nature to surround a septic inflammation with a non-infective or simple, protective inflammation. As the simple phlebitis or arteritis leads to thrombosis and ocelusion of the lumen of the vessels, hæmorrhage on account of an appendix abscess opening the iliac vein or artery is uncommon, yet not unknown. A few cases of hæmorrhage, usually fatal, have been reported.

Thrombosis may follow operation for appendicitis through direct extension of a thrombus along the deep epigastric vein to the external iliac. This is not at all a common complication, the appendix incisions seldom involving important veins in the abdominal parieties. In this it is unlike hernia incisions which are naturally the more frequently followed by thrombosis. Sometimes it occurs in the left leg from general causes.

\section{HAEMORRHAGES IN APPENDICITIS}

Homatemesis.-A number of cases of this complication of appendicitis have now been recorded by Dieulafoy, Lucas Champonière, Matheson and others. As in other things, it was the especially severe and fatal cases that first attracted attention. But now that the condition is recognized, milder cases are reported. In fine, a perfect series from coffee ground vomit to severe hæmatemesis is known. Clinically, the former are of little import, whilst the latter seem to be invariably fatal. As an example the following case may be quoted : An old man, of sixty-eight years of age, had a mild attack of appendicitis with slight fever. On the fourth day the symptoms had not settled down, so the appendix was removed. After operation he brought up large quantities of black vomit, and in a short while died. Post mortem, an acute hæmorrhagic necrosis of the mucous membrane of the stomach was found, which had spread to the submucous coat and opened the blood-vessels therein. The lesion was supposed to be due to the action of 


\section{CELLULITIS OF ILIAC FOSSE}

toxins. This case was recorded by Dieulafoy. Matheson records another in the British Medical Journal, 1901, p. 1201.

Less severe cases are improved by washing out the stomach with a solution containing about a drachm of bicarbonate of soda to the ounce of water. Our present knowledge of hæmatemesis in appendicitis must cause us to regard all cases as serious in which this symptom occurs; and it must be borne in mind that many in which it has arisen have clinically run subacute courses, and the connection between the appendix mischief and the hæmatemesis is not clear.

Homorrhage per rectum.--A few cases have been recorded in which blood has been passed per anum ; it may be liquid or black and tarry, when it is called melæna. It may arise in two chief localities, namely, in the appendix region or the hepatic flexure. In the former, it may arise by the septic process opening the vessels in the submucous layer, when the hæmorrhage may not be great, or the same process may open a larger vessel in the neighbourhood. In the latter situation, the infective agent begins its action from the peritoneal surface, i.e. from without the colon, so opening the bowel, and putting the blood vessels of the liver, etc., in communication with its lumen. In the appendix region the infection works from within out of the intestine, and at the hepatic flexure from without in. In the latter case the vessel opened is larger, and the hæmorrhage more severe. Dr. Box and Mr. Wallace (Lancet, 1903, i. 1588) describe a most interesting and fatal case, in which two severe hæmorrhages occurred per rectum. Owing to the suppurative processes in both the cæcal and hepatic regions of the colon it was not possible to identify the source of the hæmorrhage. The course of the illness was subacute, and was regarded as the earlier days of enteric fever. The cause of the bleeding is of course the ulcerative action of a septic necrosing process.

Turning attention to other infective diseases such as malignant fevers, it is known that in them hæmorrhages occur under 


\section{SURGERY OF THE APPENDIX}

the mucous membranes; and if these are put into communication with the interior of the bowel melæna results. A similar condition may be present in appendicitis and may explain the origin of the bleeding at other points than the cæcum and hepatic flexure, places where we know that abscesses frequently occur.

Finally, it may be pointed out that Lilienthal has drawn attention to the concomitance of hæmorrhages from the bowel with torsion of the appendix, page 69 .

As in malignant fevers, other hæmorrhages than those by mouth and anus are known, although in appendicitis their occurrence is but rare. Petechiæ under the skin have been described, hæmorrhages from the uterus, from the kidney, and so forth. That from the uterus is of some slight importance in connection with a possibility of extrauterine fœtation and that from the kidney with renal calculus.

Secondary hæmorrhage may occur after operations for acute infective disease of the appendix, and is most common after appendix abscess. In a case recorded by Ramsay in the Lancet, 1903 , i. 1590, a part of the external iliac artery sloughed and gave rise to severe hæmorrhage on the ninth and eleventh days after the abscess had been opened. The external iliac artery was ligatured transperitoneally. The boy recovered.

Examples of severe hæmorrhage have also arisen from the septic process opening up the iliac vein. Dr. O'Connor, of Buenos Ayres, records a fatal case of secondary hæmorrhage, most probably from the external iliac vein, in the Lancet of August, 1902. Vessels in the abdominal wall occasionally give rise to secondary hæmorrhage, but this is comparatively unimportant.

\section{CHRONIC CELLULITIS OF THE RIGHT ILIAC FOSSA}

An extremely interesting condition is seen sometimes and results from appendicitis. There is a previous history of pain, 
etc., never acute, and there has been a mass in the right iliac fossa for some months or a year. On examination a large swelling is felt, usually very hard, and giving the impression of being a large malignant growth of the cæcum; or at other times it seems of stony hardness and so fixed to the ilium that it is frequently regarded as a sarcoma of the pelvis. Yet alternative diagnoses may be made, actinomycosis abdominis, tubercular disease, or gumma.

Reaction to iodide of potassium is slow if marked at all. On exploring the mass nothing but granulation tissue is found, and no streptothrix can be cultivated. The mass slowly subsides. These cases appear to be chronic cellulitis, but whether they are due to disease of the appendix there is no positive evidence, only probability. Apparently the most difficult thing to exclude is actinomycosis and its relations.

\section{MYOSITIS OF THE ILIACUS AND PSOAS}

If the inflammation spreads beyond the cellular tissue over the iliacus and psoas, these muscles will become affected with a myositis which may be simple or septic. In some cases the infection may be so virulent as to lead to sloughing of part of the muscle. When these muscles are irritated the thigh becomes flexed, but when the muscle substance is destroyed by suppuration there are no signs that indicate its involvement, which will only be discovered at the operation.

When the myositis has been chronic, fibrous tissue is formed between the muscle fibres, and by its contraction tends to kill them. Chronic myositis cannot make much headway in an active muscle, by whose own contractions are brought about those changes which we try to produce by massage.

In a similar way the lower nerves of the lumbar plexus, especially the anterior crural, become inflamed, as may be seen clinically by pains, etc., along the branches of the nerve in the thigh and leg. And it may be suggested that in the after his- 


\section{SURGERY OF THE APPENDIX}

tory of cases of appendix abscess, the fibrosis of the nerves may explain many of the pains complained of in the iliac fossa and lower limb, which are usually regarded as functional. Wasting of the limb has never been described.

\section{PAROTITIS}

Inflammation of the parotid has been known for a long time to occasionally follow abdominal section, and other operations involving injury to the peritoneum. Clinical experience shows that it is most likely to occur when the disease of the abdomen is a septic one. For instance, it seems more common after operations for abscess and peritonitis than after clean ones like appendicectomy in a quiet interval. The causation of the disease is obscure. Yet on the whole one is inclined to associate it with some infection along Stenson's duct from the mouth, knowing the difficulty of keeping the mouths of these patients clean. It usually occurs a few days or so after operation : first on one side, followed rapidly by involvement of the other side, but may occur in patients with appendix abscess where no incision has been made. In some cases both sides seem to start simultaneously. It is impossible to say which case is going to suppurate and which to resolve. The onset is accompanied by some rise of temperature, headache, malaise, pain in the region of the ear, increased by swallowing and opening the mouth, and rapid swelling about the neck of the lower jaw. Hot fomentations relieve the pain considerably, but an incision should be made as soon as the presence of pus is suspected, and several may be required as the suppuration is very often diffuse.

In the Clinical Societies' Transactions for $1900 \mathrm{Mr}$. Bland Sutton has recorded a case of staphyllococcus infection of the parotid, following a local infection of the abdomen with the bacillus coli communis. The different infections may indicate different sources of origin. 


\section{PAROTITIS}

If the parotid suppuration is intense the pus may be discharged by the ear, although other openings have been made and the possibility of some necrosis of the lower jaw must be remembered if the sinuses do not heal.

This variety of parotitis, not being due to the specific organism of mumps, shows no tendency during its course to involve the testis.

In a recent communication, Mr. Brennan Dyball ${ }^{1}$ of Exeter has recorded a fatal case of parotitis following acute appendicitis, and has suggested that a non-suppurative inflammation of the glands may arise from the action of toxines in the blood. When suppuration occurs, he suggests that it is due to infection from the mouth.

1 Brit. Med. Journal, April 30, 1904. 
Complications of Appendicitis (Contd.): Pelvic Complications : Salpingitis, Pyosalpynx, PregnancyIntestinal Obstruction-Fistule (External and Internal)-The Relation of the Appendix to Hernia-Effects of the Toxines-HypochonDriasis-Neurasthenia - Rheumatism and GoutSyphilis and Gonorrhoa-Appendicitis with Dysenteric, Typhoid and Stercoral Ulcers

\section{PELVIC COMPLICATIONS}

Is appendicitis the infection not infrequently extends to the pelvis, affecting the pre-rectal pouch of the peritoneum. And it is undoubtedly responsible for by far the majority of cases of right-sided pelvic peritonitis. An appendix abscess frequently reaches to the pelvis and forms a loculus there. In connection with this one cannot help being struck with the possibility that disease of the appendix may be primarily responsible for many cases of right-sided salpingitis, especially those with foul-smelling collections of intra-peritoneal pus, in which the tip of the appendix is usually found intimately matted with the adhæsions. The exact inter-relation of appendicitis and salpingitis is not understood completely, but the former may be regarded as the primary source of the trouble in many cases of salpingitis, suppurative or non-suppurative, especially in young adults and up to the age of thirty, and when the disease is on the right side of the pelvis. The connection between the inflammatory affections of these two organs is such that the rule may be laid 


\section{PELVIC COMPLICATIONS}

down that the appendix should be examined, and probably removed, in every case of right-sided salpingitis or pelvic peritonitis which comes to operation. The converse that the right tube should be examined in the operation for appendicitis does not seem to be so generally applicable. Still it is a good line of practice to follow.

The connection between the lymphatics of the appendix and those of the right ovary and broad ligament, viâ the appendiculo-ovarian ligament, emphasizes the inter-relation of diseases of these structures; and it is an open question whether the "ovarian" pain complained of in cases of chronic appendicitis may not be due to involvement of the ovary viâ these lymphatics. In more acute cases, suppuration from the appendix may follow the pelvic cellular tissue, the case appearing to be one of ordinary pelvic cellulitis.

Hermes ${ }^{1}$ has recently given his experience of the condition of the appendix in seventy-five consecutive gynæcological operations, and found that organ diseased in 53 per cent. A second point he makes is, that the disease is found much more frequently in multiparæ than in primiparæ.

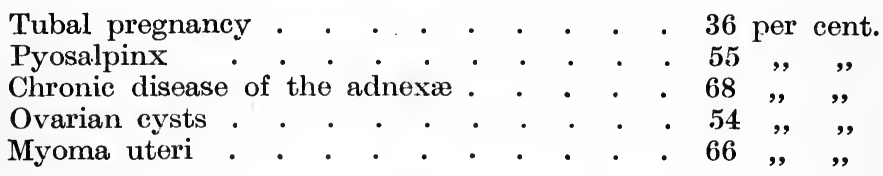

The cases are naturally either due to the pathological process beginning primarily within the appendix or secondarily as the result of disease from without. This is a very difficult question to decide, and considering the extreme frequency of disease of the appendix, it seems reasonable to regard it often as a concurrent affection, less commonly as the primary focus, and perhaps least frequently as being affected secondarily by pelvic disease. Hermes thinks that the appendix was affected

${ }_{1}$ Deutsche Zeitschrift fur Chirurgie, 1903, lxviii. Hft. 3 and 4, p. 191. I 83 


\section{SURGERY OF THE APPENDIX}

from within itself in 27 per cent. of the cases in which changes were found in it.

Pregnancy is a very important condition that is sometimes associated with appendicitis. In itself it will, however, have little effect upon the incidence of an attack. The latter is, however, a very serious complication, as it frequently leads to abortion, miscarriage or death of the fœtus. This, however, is not always the case. Sometimes the attack subsides, and is followed by the delivery at full term of a living child. Instances too have been reported where the appendix has been successfully removed without disturbing the pregnancy, and such have been under our care. Similarly, an appendix abscess has been opened and the pregnancy has not been interfered with. Of course in the latter case abortion or miscarriage is far more likely to occur than in the non-suppurative attack. Cases of peritonitis associated with pregnancy have a very bad prognosis. It may indeed be urged that the fact of a woman being pregnant forms an additional reason for early operation; or in the present state of opinion in England it forms a further reason for the practitioner to advise early operation. In this way there will be better results for both mother and child than can be obtained by the old treatment of waiting until operation is only too plainly demanded.

It is by no means uncommon for a pregnant woman to complain of pain in the right iliac fossa which is indistinguishable in its character and concomitant symptoms from mild attacks of appendicitis. A certain diagnosis is very difficult to make and occasionally the sufferings are sufficient to cause the woman to ask for operation. The state of the temperature will form an important help in coming to a right conclusion as to the meaning of the symptoms. Sometimes the appendix may not be found grossly diseased, and it seems possible that the pain may have been caused by stretching of the lymphaties in the 


\section{INTESTINAL OBSTRUCTION}

appendiculo-ovarian ligament, by the growth of the pregnant uterus, the lymphatics having been inflamed previously as a result of chronic disease of the appendix. Operation relieves the tightness of the ligament and removes the danger of the incidence of an acute attack of appendicitis. This procedure is by no means necessarily followed by abortion. In fact, operation seems to have less risks to the pregnancy, the mother and the child, than the disease has.

Acute appendicitis with peritonitis is extremely fatal to both mother and child.

\section{INTESTINAL OBSTRUCTION}

Intestinal obstruction is a common and most important com. plication of appendicitis. During a quiet period, when the inflammation may be inactive and long past, it may arise by an adherent appendix ensnaring a loop of small intestine, from stricture of the gut, adhæsions or bands. One of the most common ways is that the terminal few inches of the ileum become bound by adhæsions to the pelvis, thus establishing a series of imperfect obstructions. Sooner or later the bowel above gets distended, and through its own internal pressure pulls and perhaps twists upon the highest adhæsion, superimposing an acute attack on the previous chronic condition. Obstruction may occur in several ways in cases of acute appendicitis, especially if previous attacks have been experienced. By far the most frequent cause of obstruction in these acute cases is paralysis of segments of the small bowel, due to inflammation and perhaps the action of toxines upon the muscular layers. Neither of these classes of case is overlooked on account of the acute symptoms, but unfortunately there exists a most insidious group which, for want of a better name may be called post-operative obstruction. It must be at once explained that the operation performed has in all probability nothing whatever to do with the consequent obstructicn. 


\section{SURGERY OF THE APPENDIX}

These misfortunes arise most frequently after cases operated on for appendix abscess, but the authors have seen them occur after operations for appendicitis with general peritonitis, and once after a simple appendicectomy in a quiet period. In almost all cases the obstruction is due to small gut becoming distended and kinking over an adhæsion. Unfortunately, the diagnosis is tardily made, the surgeon's mind being more full of ideas of peritonitis than obstruction.

The time during which obstruction of the bowels may come on as a result of the formation of adhæsions will vary much in different cases. Urgent symptoms from matting of the intestines may develop in a few days, or it may be months before any complaint is made. It is in early cases rather tempting to separate recent adhæsions with the finger and so release the bound coils, but this requires careful manipulation, for it is easy to tear off sections of the wall of the bowel, which may do irrevocable damage, leading to fistula, or more firm adhæsions, and a return of the obstruction. We are of opinion that often the wisest plan is to perform a short circuit above and below the obstructed length of bowel, especially when time is of importance and operation must be quickly performed.

Again, operation is delayed as conviction comes late, and also because no surgeon is eager to treat cases of secondary peritonitis by open section, knowing the bad prognosis. In many instances the time for operation is allowed to pass by, and treatment by saline purgatives, infusions, or perhaps morphia is pursued. Few more difficult cases are to be met with in which a decision must be formed and acted upon right speedily.

The diagnosis between appendicitis with peritonitis and intestinal obstruction is by no means easy. In well marked examples the differentiation may be simple. Thus in one case there is the distended soft belly with visible peristalsis, and in the other there is a hard tender belly with no visible peristalsis. A little appreciated clinical fact may be mentioned here. If 
notice is taken of the sensitiveness of the abdomen to palpation and percussion in the two cases, it may be frequently noticed that in obstruction palpation is most complained of, and in peritonitis percussion.

Speaking generally, the more subacute the clinical varieties are, the more easily are they separated; and the more acute. the more inseparable are they for diagnostic purposes. In looking back over a series of 400 acute abdominal cases, the most frequent mistake which practitioners fell into was the mistaking of peritonitis for obstruction. Both begin with the symptoms which constitute peritonism, i.e. shock, collapse, abdominal pain, vomiting, etc. In both, the bowels may be absolutely inactive, but beyond this, in peritonitis, there is inflammation, usually with rise of temperature. Until the disease is well marked its causation can merely be guessed. Later, when in peritonitis the poisoning produces a torpid mental condition, the abdominal tenderness and rigidity pass off, and the case looks like one of obstruction. Yet post mortem a pint or more of offensive pus may be found in the peritoneal cavity in such a case !

After an operation, care and caution cannot be exercised too much in the study and treatment of cases in which unfavourable symptoms occur. Patients react so differently to the various types of infection. The pulse, temperature and abdominal conditions help little owing to the tenderness consequent on the presence of the wound. Two points are of great import: one, the persistence of the vomiting; two, the continuous increasing rapidity of the pulse. We know that the most alarming post-operative symptoms may subside if the bowels are freely open. And consequently it is now that a diagnosis must be made between what may be called conditions demanding, and conditions not demanding operation.

The possibility of the occurrence of peritonitis as a complication of intestinal obstruction must not be forgotten. In a cer- 


\section{SURGERY OF THE APPENDIX}

tain group this can be recognized. The patient is usually suffering from a malignant growth of the large bowel, which has given rise to evidence of its presence during a varying period, usually some weeks-(abdominal pains and constipation, etc.) - then there has been a change, vomiting with an increase of the pain coming on without apparent reason, distension of the abdomen with acute tenderness, which is so acute that the patient almost jumps in bed when the abdomen is percussed. Here there has either been an extension of the ulceration of the growth to the peritoneum or the perforation of a stercoral ulcer.

\section{FISTULAE, EXTERNAL AND INTERNAL.}

Fistulæ which result from disease of the appendix may be of two kinds: external when they open on the skin, internal when they open into one of the hollow viscera. The external fistula is quite a common complication after operation on an appendix abscess, but closes spontaneously in a few days, and in truth causes far more discomfort than disaster. There seems to be a good deal of misapprehension as to the origin of these fistulæ, so that it is advisable to briefly discuss the matter.

Fistulæ may arise from the perforation of the appendix wall, which had very likely given rise in the first place to the formation of the abscess. This method has been regarded popularly as the common one in cases where the appendix has not been removed. In another class of case in which it has been removed, imperfect closure of the cæcal stump of the appendix was considered the raison d'être. And so long as surgeons were afraid to explore and examine appendix abscesses, so long did these $\grave{a}$ priori suggestions of origin last. In exploring a number of appendix abscesses, it was found that the necrotic process in the appendix had not unfrequently led to sloughing of part of the wall of a coil of the small bowel contiguous to it. 
A fistula was thus formed by direct extension of the inflammation. Occasionally a communication is made with the cæcum, but from a series of consecutive cases the proportion in which the small gut and cæcum were affected was about three to one. Operation on cases of persistent fæcal fistula led to a similar conclusion. The circuitous route which may be taken by a fistula following an appendix abscess is deserving of notice. The usual place for them to open is in the right iliac fossa, but they have been found extending to the ribs, to the umbilicus, the left side of the abdomen, and even communicating with a left empyema!

More than once a fæcal concretion had been overlooked as it escaped from the appendix and has been found in or at the bottom of a sinus. We have already given interesting examples of such occurrences after localized suppuration.

Internal fistulæ are formed in two ways : either by a less acute process which allows of adhæsion of the viscera, and then perforation of the intervening septum, or by the discharge of an appendix abscess into a viscus, but in almost all cases the tract quickly closes, and the fistula is rarely seen. The most common viscus for the appendix to be connected with is the ileum, then the cæcum. Rarer communications are with the bladder, vagina, and rectum.

So far as our knowledge goes, the cases do extremely well, the fistula almost invariably closes spontaneously and causes no further complication. Extremely rarely the appendix has been known to communicate with the ureter or pelvis of the kidney.

The treatment at first consists of rest, diminution of the amount of fluid taken by the moutb, syringing of the wound with some bland deodorant such as creolin, protecting the surrounding skin, if necessary, with a heavy ointment such as unguentum cetacea, or better, covering it with melted paraffin. The paraffin should melt at a temperature a little 


\section{SURGERY OF THE APPENDIX}

above that of the body, so that it forms an easy soft layer which does not crack. This last method has been found the most valuable.

If the fistula will not close, or after a good long time, such as six months or a year, has elapsed still discharges, further operation must be considered. The trouble with the surrounding skin and the patient's general condition may demand earlier intervention. Operative treatment consists of two chief methods : in one the edges are pared, the mucous membrane inverted, and the parieties closed. This unfortunately frequently breaks down, and is unsatisfactory owing to the septicity of the locality. The more radical method consists of boldly opening the abdomen, excising the fistula, and drawing it out with the bowel attached; the gut may now be freed, sewn up, and the belly wall closed. This method yields the better results, but carries with it perhaps a graver risk, of peritonitis.

Intermediate methods of treatment are to be condemned, and are likely to lead to disaster. Simple scraping of the fistula is usually unsatisfactory. Others with very long tracts down into the iliac fossa, need a counter opening in the loin. Some of these fistulæ seem to lose their communication with the bowel, yet may be most obstinate in closing.

Before dealing with these cases care must be taken to exclude actinomycosis and tubercle, for in them the abdominal contents may be so matted that the fistula is a mere part of the disease, and death from intestinal obstruction has occurred as a result of closing the fistula $!^{1}$

\section{THE RELATION OF THE APPENDIX TO HERNIA}

The appendix may be found under several conditions in a hernia. Firstly, it may be the sole viscus in the sac ; secondly, it may be present along with other organs, usually the cæcum,

${ }^{1}$ For further consideration of the subject of fistula and its consequences, see The Erasmus Wilson Lectures for 1904 (Constable \& Co.). 
also with small bowel or omentum. It may then be present in conditions reducible, irreducible, inflamed, or strangulated. The obstructed or incarcerated form of hernia is not recognized, but is one upon which considerable stress will be laid later. As regards the anatomical situation of the hernia, it is relatively much more commonly found in a right femoral hernia than in a right inguinal, rarely in a left inguinal or femoral. In all these situations it may be found alone, but more commonly, especially in the reducible or irreducible forms, along with other viscera. In umbilical herniæ it always occurs with other viscera. Women are relatively the most common subjects, and children, especially babies, most frequently exhibit the left-sided hernia. It is a most interesting fact that in the reducible and irreducible forms it is practically always present along with other viscera, whilst in the acute examples of inflammation or strangulation it is most commonly alone. Cases in which it is pulled into the wound during the freeing of the sac at operation must be excluded.

It is difficult to understand that an appendix can be strangulated at all. With the presence of other viscera it may become strangulated along with them. But when present alone it is extremely hard to conceive such a condition unless the organ is extruded from the abdomen through a comparatively small ring or into a hernial sac just large enough to contain it tightly. In no other way, excepting by the slow contraction of the tissues round the neck of the sac, when it is by itself, can the appendix become strangulated. Yet there is the paradox that it is almost always alone in the cases described. And the question resolves itself into the search for an explanation. The key to the situation is readily found. When in a hernial sac, especially a femoral, the appendix is liable to frequent slight injuries which result in more or less chronic inflammations, so that from interference with its peristalsis and perhaps adhæsions, it can no longer properly empty itself. 


\section{SURGERY OF THE APPENDIX}

In consequence, the organisms within it grow luxuriantly, the appendix becomes thickened and more constricted at the neck of the sac; and sooner or later an acute attack is precipitated. The organ when removed shows appearances similar to those had it been strangulated, namely, there is a line of constriction with the distal part of the organ discoloured, the colour being sharply delineated at the line of constriction. But there has been no organic obstruction to the lumen, but only a functional obstruction. It may then be stated that these cases are really appendicitis in hernial sacs (see frontispiece).

When intestine is present in the sac, the appendix, by forming adhæsions or knots, may strangulate the bowel in the same way that it does within the abdomen. These cases are rare, but not unknown ; and may be suspected in that the hernia, apparently strangulated and giving rise to acute obstruction, has an impulse on coughing, and should therefore be either incarcerated or obstructed, unless the condition is one of intestinal obstruction within the hernial sac.

The diagnosis and general treatment need little comment; and the only point of special interest is what to do with an appendix found in a hernia sac. The answer to this question is, without doubt, let it be removed. This can be done in both inguinal and femoral regions, and even age-with the use of the quick clamp method of amputation - need be no contraindication. This method is aseptic and there need be no fear of infection of the wound and subsequent failure of the radical cure. ${ }^{1}$

\section{EFFECTS OF THE TOXINES}

Our knowledge of the effects of the toxines of appendicitis leaves much to be desired, and has been very little investigated. Their various reaction upon the different individuals seems to be considerable. It is a striking fact that great differences are seen in the mental condition of cases suffering from peritonitis

${ }^{1}$ Corner \& Spurrier, St. Thomas' Hospital Reports, 1902. 
of similar clinical, and possibly bacteriological, severity. For instance, one patient was dull, apathetic, and nearly comatose, whilst another, a fat publican, refused all treatment until next day when he had talked over his affairs and his will with his solicitor, a proceeding shortly followed by death. In other patients the mental condition is little affected until near the end, when they become maniacal. This subject opens up a large field of mental affections which it is not desirable to discuss in this place. We shall confine ourselves to certain known bodily ills.

Dr. Brewer, in the Annals of Surgery for 1902, vol. 36, p. 481, records a most interesting case which occurred in a boy of twelve years of age, who was recovering from an attack of appendicitis with spreading peritonitis. Up to the third night, progress was most satisfactory, when he suddenly became delirious with attacks of shrieking. Later a sweetish odour was noticed about the breath. Acetone was found in both the blood and urine. The paroxysms were succeeded by coma and death. Dr. Brewer seems to think that the action of the anæsthetic had some part in the production of the acetonæmia, a condition seldom seen apart from diabetes. On the other hand, it might be brought about by an alteration in the metabolism of the liver by toxines brought to it from the bowel or peritoneal cavity.

In 1903 Dr. Ballin, of Detroit, reported a case, and collected some more from the literature in which the toxines had brought about acute yellow atrophy of the liver. In these cases there is a great interference with proteid metabolism, leucine and tyrosin appearing in the urine. The relation of the presence of these and acetone in the urine is not understood. In his paper, Annals of Surgery, 1903, cxxiii. p. 362, he collected ten cases of post-operative yellow atrophy of the liver ; two of these followed appendicitis, four other abdominal operations, and another a filthy condition of the mouth in which the 


\section{SURGERY OF THE APPENDIX}

toxines would be absorbed from the alimentary canal. It may. be assumed; therefore, from these cases that the functions of the liver are, rarely, very seriously, upset, and in all probability there exists all degrees or grades of interference with the metabolism of the liver. On the one hand, every one has noticed jaundice in varying degree in these cases as seen in the tinged conjunctivæ, and on the other, Dr. Brewer tells of a fatal acetonuria, and Dr. Ballin of acute yellow atrophy of the liver.

The obvious treatment of such toxic jaundiced cases is by purgation, and every surgeon knows the vast importance in all abdominal work of this procedure in draining off intestinal toxines.

In one case, that of a young married lady who had been operated on by one of us for early perityphlitic abscess, acute jaundice, with delirium and increasing coma, rapidly proved fatal, the patient dying within a week after the operation. The local condition presented nothing unusual and was progressing very satisfactorily. No necropsy was permitted, but it was the opinion of Dr. Harper, under whose care she was, and also of the operator, that the fatal complication was acute yellow atrophy of the liver.

\section{HYPOCHONDRIASIS OR NEURASTHENIA.}

Such a state of mind may be consequent upon an attack of appendicitis or merely the fear of it. The unhealthy mental condition is maintained and increased by introspective searching as to the causation and significance of certain sensations, probably movements of peristalsis, and especially so if they can be located in the right iliac fossa. Hypochondriasis and hysteria, or better by far, neurasthenia, with reference to the abdomen have become more frequent since appendicitis has become such a common and also fashionable complaint.

The state is as a rule easily recognized by merely listening to the tale of self-inquiry and self-examination, but much more 


\section{HYPOCHONDRIASIS}

certainly is it shown in the extraordinary deductions made by the patient on account of his exaggerated opinion of the importance of his symptoms. Having thus formed an opinion of the mental condition of the patient, the next thing to do is to inquire into his bodily condition. Unfortunately, he cannot help us to form so certain an estimate as to the state of things in his iliac fossa as in his mind. It is often impossible to discover anything by abdominal examination on account of the great thickness of the abdominal wall in many cases of this type of neurasthenia and the hindrances to that examination caused by the patient's emotions. Greater reliance can be placed on a rectal (or vaginal) examination, a procedure frequently more easily and more satisfactorily made than the abdominal, as the patient does not understand its relation to the disease from which he considers himself to be suffering. And in this way one may guard against condemning a patient as merely neurasthenic who has a chronically inflamed appendix in the pelvis. Still, in many cases it is impossible to exclude disease of the appendix, and recognizing that this organ is known to be frequently chronically inflamed and fibrous, so being incompetent to empty itself by peristalsis and as a consequence giving rise to symptoms of dyspepsia, constipation, colitis, general depression, etc., which condition, often unrecognizable objectively at the bedside, may be the "fons et origo mali" of the neurasthenia; or, perhaps better, the chronic appendicitis may have been the cause which turned the neurasthenic's attention and inquiries to his right jliac fossa. Remember also, that very frequently at the bottom of a case of "hysteria" lies some slight disease which the patient's state of mind exaggerates, glorifies, and obscures.

So long as a patient reasonably sticks to his symptoms and is not satisfactorily improved with the ordinary treatment, directed with special reference to the gastro-intestinal tract, it is one's duty to recommend operation. It is an axiom in the 


\section{SURGERY OF THE APPENDIX}

science of healing that it should be a doctor's aim to remove the cause of disease if possible. In the class of cases under consideration we cannot say that there is no disease in the appendix, and there is a certain presumption that in it may be the hidden source of the patient's trouble. The course of the symptoms after operation is always difficult to prophesy, but a great source of trouble has been removed, apart from the beneficial moral effect of the procedure.

\section{RHEUMATISM AND GOUT}

There is doubtless a connexion between the former disease and appendicitis. This has been proved clinically over and over again by their association, the action of salicylates, and so forth. The connexion with the latter disease has not been so prominently put forward. Dr. F. J. Poynton, in 1900, read an excellent paper to the Medical Society of London on the association of rheumatism, appendicitis, and arthritis. The association of rheumatism and appendicitis was first brought forward by Dr. Sutherland in the Edinburgh Hospital Reports for 1895. Since then, a number of other cases have been noticed; but previous to this, Dr. Burney Yeo, Brit. Med. Journal, 1894, collected cases of arthritis associated with appendicitis. In these joints the presence of the bacillus coli communis has been demonstrated, but the existence of the diplococcus of rheumatism has not been. Dr. Haig regards many of these cases as manifestations of the uric acid diathesis.

By the relation of appendicitis to rheumatism in some cases the analogy between that organ and the tonsil is still further increased on account of the well known relations between tonsilitis and rheumatism. The joint troubles are secondary to the inflammation of the appendix.

\section{SYPHILIS}

The fact that syphilis can affect the appendix has only quite recently become recognized. And it may be safely stated I 96 
that it will be found to be by no means so uncommon as is thought. Attention was first drawn to this subject by Howitz in the Annals of Surgery for 1898. There he briefly records four cases of attacks of appendicitis during secondary syphilis, which got quite well with mercury. All his cases had had previous attacks, and so the syphilis may have merely aggravated the local condition. This is easily understood as the great pathological characteristic of secondary syphilis is that of a transient subacute inflammation of any organ or membrane in the body. In the case of the appendix the natural septic condition of the lumen may quite mask the syphilitic origin. Still, there is no doubt that in some cases of secondary syphilis pain and tenderness is experienced in the right iliac fossa, with slight fever, etc., and mercury seems to act like a charm.

In the tertiary stage, gummata are certainly found in this region, though their origin must always be doubtful; and the way which certain tumours in the right iliac fossa clear up under the influence of iodide of potassium seems to suggest their specific origin. Lilienthal records a very interesting case in the New York Medical Journal of February 7, 1903, p. 233, of a man aged 22, who had had syphilis and later had pain in the right iliac fossa. The appendix was removed and found to be diseased and cystic. The wound refused to heal, developed the appearance of a gummatous ulcer, yet rapidly cicatrised when iodide of potassium was administered. Of course it is doubtful if the appendix was gummatous, though the suggestion is feasible.

\section{GONORRHEA}

It has never been proved that a case of appendicitis has been of gonorrhœal origin, though in a case observed by one of us, three diseases-gonorrhœea, appendicitis, and arthritis, co-existed.

In many text-books it is asserted that gonorrhca may 


\section{SURGERY OF THE APPENDIX}

give rise to peritonitis. In the female this is easily understood, but in the male the disease appears to have had its origin in an inflammation of the appendix.

\section{APPENDICITIS WITH DYSENTERIC, TYPHOID AND STERCORAL ULCERS}

Appendicitis is very liable to follow upon any infective diseases of the intestinal tract, such as typhoid, dysentery, etc., and may be produced either by the specific organisms or the septic ones which are associated with them. Thus the appendicitis may be in no way different from an ordinary attack because it occurs in the course of typhoid fever, dysentery, etc. In cases in which the large bowel is full of stercoral ulcers, the constipation and mucous formation lead to the growth of organisms which may produce an appendicitis. In point of fact the most frequent situation to get an abscess in these cases is in the right iliac fossa, and although the ulcer in the cæcum communicates with the abscess it is not always certain that this may not be secondary to an appendicitis complicated by abscess. 


\section{CHAPTER XII}

\section{SELECTION FOR LIFE INSURANCE}

Tre subject of the relation of appendicitis to the selection for life insurance is a difficult one owing to the great imperfection of our knowledge of the subject. With increase of experience the fact has become known that this disease is one of the most widely spread, and the great increase of the number of cases in later years cannot be entirely explained by the fact that they were not diagnosed by our forefathers. It seems to be an undoubted fact that the disease is more prevalent now than it was twenty-five years ago. And as is always the case, it is the acute and prominent manifestations which are first recognized, and only now are we beginning to appreciate the more subacute and chronic forms with their results on the system. Generally speaking it is the incidence of the acute forms which is of the most interest from the point of view of insurance, as the chronic affect the health and power of usefulness rather than take the life of the individual.

It is most obvious to all who have kept the registers of large hospitals that it is the first attack which is responsible for at least three-quarters, if not more, of the fatal cases of appendicitis. And insurance companies cannot protect themselves against first attacks ; the risk must be covered by the ordinary premium. The mortality of the cases in St. Thomas' Hospital for the years 1894-1901 inclusively was, for cases in the first attack, 25 per cent. ; in their second, 7 per cent., and in their third, 2 per cent. These figures only represent such cases as were sufficiently severe to necessitate the removal 


\section{SURGERY OF THE APPENDIX}

of the case to hospital, and do not therefore by any means represent true mortalities. But they do show quite clearly the difference in the mortalities of the different attacks of the disease. General peritonitis, in three-quarters of the cases, arises in a first attack, and a localized abscess usually in a later one. As companies cannot protect themselves against first attacks, unless they take regard of the ages 10-30, by far the greatest period of incidence of acute fatal cases, and give special premiums, it is chiefly necessary to discuss those who have had one or more attacks. These fall into two groups, i.e., those that have not been operated upon (medical), and those which have (surgical).

1. Cases which have not had operation.-The first question to arise is, what are the prospects of further attacks? This is a very difficult point, and has been very variously estimated. Writers have distinguished, or tried to distinguish, between "relapses" and second attacks. The result of so futile an attempt has been to mix matters considerably. Lennander heads the list in saying that all cases have further trouble. And this seems the more likely to be true if the wider view is taken, namely, that an appendix once diseased is likely to be maimed, and consequently cannot fulfil its functions, becoming a culture tube for the growth of organisms and unable to empty itself. The results of an attack may be other acute or subacute attacks, chronic appendicitis, appendicular toxæmia, typhlitis, colitis, and so forth.

TABLE OF THE VARIOUS ESTIMATIONS OF THE FREQUENCY OF FUTURE ATTACKS.

Fowler, Barton, Richardson

\section{Porter}

Ranzhoff

Bryant

Sahli

Krafft

Kuanssold

Hawkins

Witt further attacks 8 per cent.

$\begin{array}{lrrrr}, & , & 10 & , & , \\ , & , & 13 & , & , \\ , & , & 20 & , & , \\ , & , & 22 & , & , \\ , & , & 23 & , & , \\ , & , & 24 & , & , \\ , & , & 30 & , & ,\end{array}$




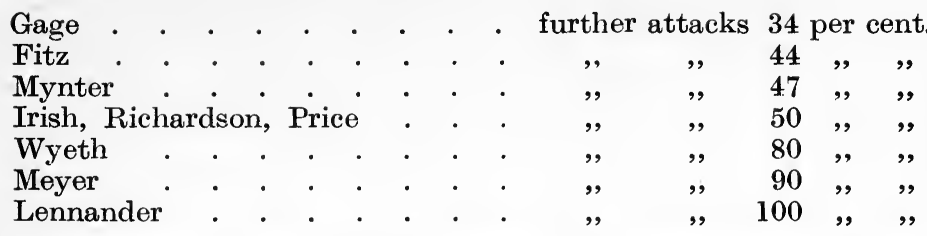

These immense variations indicate the impossibility of forming a definite opinion and for selection in insurance work. The time which has elapsed since or between the attacks is of more importance, and the mild attacks and symptoms i.e. "appendix trouble," which many of the above authors have included, need not be considered. Thus of recurrent attacks which are likely to affect life, 50-60 per cent. occur in the first six months, 80-90 per cent. within the first year after a definite attack.

The details recommended to be considered are-

1. The number of attacks;

2. The dates and approximate durations of attacks;

3. Their severity, as estimated by the length of time in bed, the vomiting, etc.;

4. The presence of tenderness at the time of examination;

5. The general health and the condition of the gastrointestinal tract.

In all cases there should be an extra premium where the proposer has had a recent attack which was evidently one of appendicitis.

Cases should be acceptable after two years of immunity from symptoms after one attack, at ordinary rates. In relapsing cases, i.e. with more than one attack, five years should elapse. Those cases where the later attacks are becoming milder and of shorter duration, can be accepted freely after a period of five years with total absence of symptoms.

2. Cases which have undergone an operation.-Many examples must be considered under this heading, and naturally somewhat complicated factors must be taken into account, 


\section{SURGERY OF THE APPENDIX}

chiefly with the view of post-operative complications on which an opınion must be given.

(a). Any subject who has undergone a satisfactory removal of appendix may be accepted six months after the operation.

(b). Cases of suppurative appendicitis with the formation of a local abscess, and in which the appendix has not been removed, would be eligible for acceptance after two to three years of immunity from trouble, with the addition of a moderate extra premium.

(c). Cases of suppurative appendicitis with the formation of a local abscess, and in which the appendix has been removed as well as the abscess drained, may be accepted after six months to one year of freedom from trouble. Dr. Miles F. Porter gives the percentage of recurrent attacks in cases of local abscess in which the appendix has not been removed as $13 ; 102$ cases were examined. In our belief this percentage should be much higher, and very much more so if chronic appendicitis is taken into account.

(d). Cases of spontaneous bursting of the abscess, and closure of the sinus without operation, should have at least three years allowed them before acceptance.

(e). Cases of recovery after local spreading peritonitis may be accepted after six months; cases recovering after diffuse peritonitis should not be accepted within eighteen months to two years, the appendix having been removed in each case. If this has not been done a period of five years should be allowed to elapse.

In the above, it will be seen that great stress is laid upon the removal of the appendix. This is most important, because acute and subacute "recurrent" attacks are probably a great deal more frequent than is believed. And even if these are not noticed, chronic appendicitis and its attendant evils are almost certain to ensue.

All operative cases should be examined for fistula, sinus, 


\section{LIFE INSURANCE}

and, above all, ventral hernia. None of these need exclude the subject from acceptance, but all would demand a special and substantial addition to the premium. There is an important post-operative complication against which little protection can be taken, namely, intestinal obstruction. As a general rule this more commonly follows suppurative than non-suppurative appendicitis, and is most likely to declare itself within the times advised to elapse before the acceptance of the life, than afterwards. 


\section{INDEX OF NAMES}

Ackermann, 157

Addison, 21

Andral, 145

Anteirieth, 23

Bäarheim, 165

Ballin, 193, 194

Banks, Sir W. Mitchell, 96

Barker, A. E., 83

Barnard, 64

Barth, 166

Barton, 200

Battle, W. H., 23

Beck, 76

Beger, 162, 163

Berg, 125

Bergmann, 166

Berry, 16, 27, 28, 29

Bierhoff, 159

Birch-Hirschfeld, 159

Bishop, 102, 157

Blatchford, 23

Böstrom, 165

Bouglé, 166

Bowlby, A. A., 80

Box, C. R., 87, 170, 171, 177

Boxall, 70

Braun, 166

Brewer, 193, 194

Broca, 69

Bryant, 161

Caussade, 56

Chaffey, 157

Champoniere, Lucas, 176

Clado, 21

Copland, 14

Corner, 157, 160, 172, 190, 192

Cullen, 163

Cunningham, Prof., 21, 22

Daske, 165

Daunic, 164

Davis, 160
Dawbarn, 69, 82

Deaver, 13, 50, 53, 55, 83, 159 , 161

Dillard, 24

Dieulafoy, 56, 176, 177

Dixon, 30

Dodwell, 166, 167

Draper, 163

Duplant, 165

Dyball, Brennan, 181

Eccles, 165

Ekehorn, 165

Elliott, 75

Elting, 163

Enderlen, 157

Fairbairn, John, 164

Fairbank, H. A. T., 160

Fawcett, Prof., 23

Fenwick, Dr. Samuel, 111

Fenwick, Dr. Soltan, 166, 167

Fischer, 75

Fitz, 14, 167, 201

Forster, 14

Fowler, 75, 200

French, 55

Gage, 201

Gangolphe, 165

Gerster, 121

Gilford, 159, 160

Girard, 164

Glazebrook, 159, 160

Goffe, 164

Grill, 165

Grisolle, 14

Guder, 165

Haasler, 157

Haddaeus, 166

Haggard, 83

Haldane, 157

Haig, 196

Haller, 23 


\section{INDEX OF NAMES}

Hancock, 15

Harper, 194

Harte, 164

Hawkins, 15, 87, 89, 200

Head, Henry, 145

Heister, 14

Heller, 159

Hermes, 182

Hinglais, 165

Hodder, 162, 163

Hodgkin, 14

Hofmeister, 165

Hogarth, 157

Howitz, 197

Hunter, 23

Illich, 166

Irish, 201

Israel, 166

Jalaguier, 80

Jessup, 164

Kanthack, 159

Kammerer, 80

Keith, Arthur, 27

Kelly, A. J. O., 50, 162, 163

Kern, 165

Kolaczek, 162

Korte, 166

Krafft, 200

Kuanssold, 200

Lafforgue, 23, 158

Lange, 165

Langhans, 166

Lanz, 165

Lartigan, 164

Latimer, 165

Lee, 166

Lees, D. B., 157

Leichstenstern, 161

Lennander, 80, 201

Letulle, 162, 164

Lieberkuhn, 24, 25

Lilienthal, 69, 178, 197

Lockwood, C. B., 19, 79, 159

Lührs, 166
MacDougal, 70

McGraw, 157

McKidd, 157

Mahomed, 15

Makins, George Henry, 165

Marsh, Prof. Howard, 62

Massa, 23

Matheson, 176, 177

Maydl, 161

Meckel, 23

Merling, 163

Mestivier, 14

Meyer, W., 76, 201

Monks, 162

Montsarrat, 157

Morgagni, 23

Morison, Rutherford, 97

Morris, 14, 29, 76

Moskowitz, 163

Mosse, 164

Moullin, Mansell, 21

Müller, 161

Murphy, 14, 166

Murray, 159, 160

Mynter, 201

Nothnagel, 161

O’Connor, 97, 157, 178

Ozerung, 166

Panchet, 164

Parker, 14

Parkinson, 14

Patterson, 160

Picquand, 23

Pitts, Bernard, 157

Porter, Miles, F., 200, 202

Power, D'Arcy, 157

Poynton, F. J., 196

Price, 14, 201

Prus, 163

Ramsey, 178

Ransom, 165

Ranzhoff, 200

Regling, 162, 164

Regnier, 166

Renshaw, 157

Ribbert, 16, 29

Richardson, 14, 200, 201

Roberts, 24
McBurney, 14, 21, 23, 73, 76, 82, 97,164 


\section{INDEX OF NAMES}

Robinson, Byrom, 35

Robson, Mayo, 167

Rokitansky, 14, 163

Rolleston, 30, 154, 155, 157, 164

Routier, 69, 159

Roux, 166

Ruhrah, 165

Russell, A. E., 170. 171

Ruyter, 164

Sahli, 200

Samter, 166

Sands, 14

Santorini, 23

Saracenus, 14

Scholler, 159

Schreyer, 166

Schüller, 80

Sonnenburg, 76, 165

Spurrier, 172, 192

Stimson, 163

Sutherland, 196

Sutton, Bland, 180

Swan, 24

Symonds, Charters, 15

Tarensky, 23

Thèvenot, 165
Treves, Sir Frederick, 18, 53, 62, 72,88

Ullman, 166

Uzkow, 166

Van Hook, 82

Vassilier, 166

Vissmann, 160

Wallace, Dr., 156, 157

Wallace, C. S., 61, 177

Warren, Collins, 159, 160

Waterhouse, 157

Weinberg, 162, 164

Weir, 75, 164

Welch, 165

Westermann, 157

Whipham, 162, 164

Wilks, Sir Samuel, 15

Willson, 164

Witt, 200

Wood, 159

Wright, 14, 162, 163

Wyeth, 75, 201

Yeo, Burney, 196

Zemann, 161, 163 


\section{INDEX}

Abdominal conditions, acute, 153

Abnormalities, 30

Abscesses, 32-34, 49, 50

- subdiaphragmatic and other abscesses, 169-173

- diagnosis of, 87-89

- symptoms of, 90,91

- treatment of, 92-96

- removal after, 96-105

Absence of the appendix, 23-24

Actinomycosis, 164-166

- literature, 165

“Acute Abdomen," The, 134-153

- general description, 136-138

- points to be noted, 138-140

- requiring no operation, 140-146

Aetiology, 32-34

Age-frequency of the forms of appendicitis, 32-34

Amputation, 82-85, 97-105

Anatomy, 16

- gross, 16-19

- microscopical, 24

- surface, 21-22

Appendicitis, acute, 38-40

- apparently non-suppurative, $57-66$

- chronic, 35-38, 66-69

- obliterans, 42

- percentage of, in acute abdominal conditions, 135

- with local abscess, 44-51, 86-106

- with local peritonitis, 44-51, 106-122

- with general peritonitis, 32-34, 122-133

- tuberculous, 166-168

- with Torsion, 69

Appendicectasis, 41

Appendix, absence of, 23-24

Bacteriology, 36-39, 55-56

Benign tumours, 158-159

Capillary lymphangitis, 33

Carcinoma, 161-164
Carcinoma, literature, 163-164

Catarrhal cases, 39

Cellulitis, 39, 41, 178-179

Clamp for removal of the appendix, 83

Colic, in differential diagnosis of " acute abdomen," 141

- appendicular, 44, 69-71

- biliary, 143

- renal 143

Colitis, 37

Complications, pelvic, 144, 182185

Concretions, 51-53

- in clinical varieties of disease, 53

Constipation, appendicular, 36

Cysts, 42

Diagnosis, 61-64

- differential, 134

Dysentery, 198

Development, 27-30

Empyema, of appendix, 42

- of chest, 173

Endo-appendicitis, 39

Endothelioma, 160-161

Erysipelatous appendicitis, 39, 40 . 44

Exudation, intraperitoneal, 117

Fibrosis, 41

Fistulae, 188-190

Folliculitis, 39-40

Frequency of recurrence, 44

Gerlach, valve of, 18, 71

Gonorrhoea, 197-198

Gout, 196

Haemorrhages, 176-178

Hernia, relation to, 190-192

History, 13-15

Hypochondriasis, 194-196

Intussusception, 154-157

- literature, 157 


\section{INDEX}

Leucocytosis, 54, 147

Life insurance, 199-203

Lymphadenitis, 43, 44

Lymphangitis, 43, 44

- capillary, 39

Lymphatics, 19

Lymphoid tissue, changes in, 27, 29,36

Malignant tumours, 159-161

Meso-appendix, 16

Mesenteriolum, 43

Mortality, 134-135

Mucoceles, 42

Myositis, 43, 44, 179

Necrosis, acute infective, 44, 51

Nerves, 19

Neurasthenia, 194-196

Non-suppurative, 38, 41, 57, 58, 62

Obstruction, intestinal, 148, 185188

Operation, methods of, 68, 69, 72-85

- incisions, 72-81

- amputation of appendix, 83

Pancreatitis, acute haemorrhagic, 146

Parotitis, 180-181

Pathology, 35-66

- chronic appendicitis, 35-38

- acute appendicitis, non-suppurative, 38-44

- suppurative, 44-51

Pelvic complications, 144, 182-185

- disease differential from, 152

Peritoneal fossae, 19-21

- exudate, 117

Peritonitis, 106-114

- subacute, 43

- forms of associated with appendicitis, 48,49

- differentiation from intestinal obstruction, 146

- diffuse, treatment of, 122-133

- spreading, 115, 116

- temperature in, 119-121

- pulse in, 118-119

- treatment of, 123-133
Perforation, 45, 46, 150

Physiology of the appendix, 25-27

Position of the appendix, 17

- changes in, 29

Pregnancy, 184-185

Prognosis, 65-66

- mortality, 134-135

Recurrence, frequency of, 44

Removal after abscess, 98-105

Rheumatism, 196

Sarcoma, 159-160

- literature, 160

Sclerosis, 41, 42

Septic complications, rare,173-174

Sex, 34

Stricture, organic, 41

- functional, 41

Subdiaphragmatic and other abscesses, 169-173

Suppurative appendicitis, pathology of, 38, 41, 44-50

Surface markings, 21-23

Symptoms, 59-631

— of " acute abdomen," 136

- of onset, 138

- of peritonitis, 118,119

- that may result from appendicitis, 66, 67

Syphilis, 196-197

Temperature in peritonitis, 119121

Thrombosis, 174-176

Torsion, 69

Toxines, effects of, 192-194

Treatment, 64-65

Tuberculosis, 166-168

Typhlitis, chronic, 37

Typhoid, 198

Tumours, benign, 158

- carcinoma, 161

- endothelioma, 160

- sarcoma, 159

Ulceration, 41,44

Ulcers, gastric, 142

- stercoral, 198

- typhoid, 142

Vascular supply, 19

Butler \& Tanner, The Selwood Printing Works, Frome, and London 


\title{
PRINCIPLES OF PRACTICAL MICROSCOPY
}

BY

\author{
A. E. WRIGHT, M.D. (Univ. of Dublin) \\ Late Professor of Pathology, Army Medical School, Netley. \\ Pathologist of St. Mary's Hospital, Paddington, W.
}

Demy 8vo. Fully Illustrated with Diagrams and in Colour.

\section{ANTI-TYPHOID INOCULATION}

Containing an Exposition of the Principles of the Method and a Summary of the Results achieved by its application.

BY

A. E. WRIGHT, M.D. (Univ. of Dublin)

Pathologist to St. Mary's Hospital, Paddington.

Demy 8vo. 3s. $6 d$. net.

\section{CLINICAL CAUSES OF CANCER OF THE BREAST}

By CECIL H. LEAF, F.R.C.S.

Demy 8vo. 2s. net.

CONTENTS :

Introductory. Bibliography. Results and Conclusions. Prevention of Cancer of the Breast.

\section{LEPROSY AND FISH EATING}

BY

\section{JONATHAN HUTCHINSON, F.R.S.,}

LL.D. Camb. Edin. and Glasgow ; F.R.C.S.; late President of the Royal College of Surgeons.

Demy 8vo.

This work comprises statements as to the History of Leprosy, its Nature, its prevalence in different countries, and the conditions under which it has disappeared from many. Facts are brought forward to show that it is not ordinarily contagious, and that its real cause is the use as food of badly cured fish. It is held that the segregation of lepers is useless, and that compulsory laws are wholly unjustifiable. There are chapters on the influence of sex in relation to Leprosy of religious creed and of poverty. Some account is given of the author's tours of enquiry in South Africa, and in India, and the measures for its suppression as best adapted for different countries are fully discussed. The volume contains Maps and Illustrations. 


\section{THE LYMPHATICS}

\section{By G. DELAMERE, P. POIRIER and B. CUNÉO.}

With many Coloured Diagrams and Other Illustrations.

TRANSLATED AND EDITED BY

CECIL H. LEAF, F.R.C.S., etc.

Imperial 8vo. I8s. net.

CONTENTS :

First Part.-General Anatomy of the Lymphatic System.

General Considerations. Lymph. Leucocytes; I The White Corpuscles in General; 2 The Different Leucocytes. The Lymphatic Vessels. The Lymphatics.

Second Part.-Special Study of the Lymphatics of the Body.

Chap. I.-Lymphatics of the Lower Limb: I Glandular Groups of the Lower Limb ; 2 Lymphatic Vessels.

Chap. II.-Lymphatics of the Pelvis and Abdomen: I Glandular Groups of the Pelvis and Abdomen ; $a$ Ileo-pelvic Glands; $b$ Abdominoaortic Glands. 2 Lymphatic Vessels of the Pelvis and Abdomen : $a$ Lymphatics of the Abdominal Wall; $b$ Lymphatics of the External Genital Organs ; $c$ Lymphatics of the Internal Genital Organs ; $d$ Lymphatics of the Urinary Passages; $e$ Lymphatics of the Sub-diaphragmatic Portion of the Digestive Tube.

Chap. III.-Lymphatics of the Thorax: I Glandular Groups of the Thorax; $a$ Parietal Glands; $b$ Visceral Glands. 2 Lymphatic Vessels of the Thorax: $a$ Parietal Lymphatic Vessels; $b$ Visceral Lymphatic Vessels.

Chap. IV.-Lymphatics of the Upper Limb: I Lymphatic Glands of the Upper Limb; 2 Lymphatic Vessels of the Upper Limb.

Chap. V.-Lymphatics of the Head and Neck ; I Glandular Groups of the Head and Neck; $a$ Peri-cervical Glandular Circle; $b$ Descending Cervical Chains. 2 Lymphatic Vessels of the Head and Neck; a Lymphatic Vessels of the Cranial Region ; $b$ Lymphatic Vessels of the Face ; $c$ Lymphatic Vessels of the Facio-Cervical Portion of the Digestive Passages ; $d$ Lymphatic Vessels of the Facio-Cervical Portion of the Respiratory Passages.

Chap. VI.-Terminal Collecting Trunks of the 'Lymphatic System: I Terminal Collecting Trunks of the Supra-Diaphragmatic Portion of the Body; 2 Thoracic Duct.

LANCET.- " During the last twelve years a monumental work on Anatomy has been slowly but steadily progressing in France under the able direction of Professor Paul Poirier with the collaboration of Professor A. Charpey. . . . The section on Lymphatics . . . has now been translated into English by Mr. Leaf, and we congratulate him on having rendered the French into English with great accuracy and on presenting to his countrymen an excellent account of the present state of knowledge of the Lymphatics as it is taught and understood in France. ... This work on the Lymphatics will prove useful to the anatomist, the physiologist, and the practitioner."

Pritish Medical Journal.- “ Mr. Cecil H. Leaf's translation of the volume on "The Lymphatics' which constitutes a portion of the Treatise of Human 'Anatomy published under the editorship of Professors Poirier and Charpey, leaves little to be desired, and this was almost to be expected, for the subject is one upon which Mr. Leaf has done excellent work. . . . The first I Io pages are devoted to the general anatomy of the Lymphatic system, written by Dr. Gabriel Delamere. .... In the second and larger portion of the book Professor Poirier and Professor Cunéo describe the Lymphatic vessels and glands in the human body, and their descriptions include the results of their own investigations."

ATHEN fum.- "There can be little doubt that this book will prove a valuable addition to our medical and anatomical libraries. It has been accurately translated by Mr. C. H. Leaf. The illustrations are numerous and unusually good; many of the wood-cuts are taken from original drawings or preparations by the joint Authors. . . . To each section is appended a bibliography which must largely enhance the value of the book." 


\section{NEW METHODS OF TREATMENT}

\section{By Dr. LAUMONIER.}

TRANSLATED AND EDITED FROM THE 2ND EDITION BY

H. W. SYERS, M.A., M.D. Cantab.

Physician to Out-Patients, Great Northern Central Hospital.

Crown 8vo. $7 s .6 d$. net.

\section{CONTENTS :}

\section{Remedies Which affect the Nutritive Process.}

Nutritive Disorders. Soluble Albumins, Peptones and Albumoses. Organic Matters containing Phosphorus: $a$ Leithins ; $b$ Nucleins ; $c$ Glycerophosphates. Drugs which Initiate Indirect Modification of Nutrition : $a$ Phosphoric Acid ; $b$ Cacodylic Acid and Cacodylates ; $c$ Disodic Methylarsinate; $d$ Derivatives of Vanadium; $e$ Persulphates; $f$ Orexine. The Yeasts. Remedies whose Action is Exerted on the Organs of Bloon Formation.

The Maladies of the Blood and their Treatment. Subcutaneous Injection. Method of Linthicum. Use of Defibrinated Blood: $a$ Hæmotherapy; $b$ Hæmenteroclysis, Employment of the Blood Derivatives. Substitutes for Iron.

\section{Treatment of Minerals.}

Mineralization and Demineralization. Substances which act by Increasing the Mineral Contents of the Organism. Bouillon, Cereal Decoctions, etc. Artificial Serums. Bromated and Iodated Serums, etc. The Metatrophic Method.

Drugs Which Influence the Respiratory Function.

Elements and Factors of Respiratory Troubles. Vegetable Essences : $a$ Derivatives of Turpentine, Terpine, and Terpinol ; $b$ Eucalyptus and Eucalyptol ; $c$ Essence of Niaouli, Gomenol Creosote and its Derivatives : $a$ Creosote ; $b$ Phosphotal ; $c$ Guaiacol; $d$ Thiocol. Intra-Pulmonary Injections.

Treatment which Modifies Urinary Elimination.

Urinary Toxiclty and the Eliminative Machinery. Remedies which Act upon the Excretion of Urine, $a$ Spermine ; $b$ Piperazine ; $c$ Sidonal; $d$ Theobromine; $e$ Salts of Strontium.

\section{Drugs which Alter Vascular Tension.}

Hypertension. Tetranitrate of Erythrol.

\section{OPOTHERAPY.}

Principles of Opotherapy. Administration of Opotherapeutic Agents. Animal Matters which are made use of in Opotherapy : $a$ Thyroid Body and Parathyroid Glands; $b$ Thymus Gland. $c$ Pituitary Body ; $d$ Suprarenal Gland; $e$ Spleen ; $f$ Testicle ; $g$ Ovary ; $k$ Liver ; $i$ Pancreas ; $j$ Prostate ; $k$ Mammary Gland; $l$ Kidney; $m$ Lung; $n$ Bone Marrow; $o$ Nervous Tissue. Zomotherapy.

\section{SEROThERAPY AND VAccination.}

The Defensive Reactions of the Organism and Immunity. Antitoxic and Anti-infectious Serums : $a$ Antitetanic Serum; $b$ Antidiphtheritic Serum ; $c$ Antivenomous Serum; $d$ Antistreptococcic Serum ; e Antiplague Serum; f Anticholeraic Serum; $g$ Antityphoid Serum; $h$ Various Serums ; Vaccinations. Antirabic Vaccination.

The Nervous System and the Drugs which Act Upon it.

The Neuron and the Nervous Reactions. Recently Discovered Derivatives of Morphia ; $a$ Dionine ; $b$ Heroine ; Local Anæsthetics : $a$ Eucaines ; $b$ Nirvanine ; $c$ Orthoform.

\section{The Antipyretics.}

Fever and its Indications. New Antipyretics; $a$ Euquinine; $b$ Pyramidon ; $c$ Acetopyrin d Citrophen.

\section{The Antiseptics.}

Antisepsis. Derivatives of Silver : $a$ Argonine, $b$ Largine ; $c$ Colloidal Silver. Derivatives of Mercury : Hygrol. Derivatives of Iodine : $a$ Di-iodoform; $b$ Traumatol. Formol and its Derivatives: $a$ Formol: $b$ Iodoformine. 


\title{
THE PREVENTION OF DISEASE
}

\author{
TRANSLATED FROM THE \\ GERMAN
}

BY

\author{
WILMOTT EVANS, M.D., B.S., B.Sc., F.R.S.
}

WITH AN INTRODUCTION BY

\section{H. TIMBRELL BULSTRODE, M.A., M.D.,}

Cantab. D.P.H.

Medical Department of H.M. Local Government Board, Lecturer on Public Health in the Medical

School of Charing Cross Hospital, Honorary Secretary and Member of Council of the Epidemiological Society of London, etc.

Demy 8vo, xviii + I,063 pp. Price 3 Is. 6d. net.

LANCET.- “ . . . An important contribution towards the development of that larger conception of preventive medicine to which we seem to be gradually moving, and the medical officer of health as well as the general medical practitioner should derive inspiration from its pages."

THE DAILY NEws.--"This valuable medical work, the production in the original of a number of eminent German physicians and surgeons, is . . . the first attempt which has been made to view disease in its entirety, whether communicable or not from the standpoint of preventive medicine, in the more comprehensive sense of the term. . . Every effort has been made to render the meaning of the original into readable English, special attention having been given to rendering the scientific terms and expressions by their correct English equivalents."

Westminster Gazette.- " No one can read the book without being struck by the scope which exists for preventive operations and the very contracted character of the present day conceptions and practices in this respect. . . The subject is clearly one destined to occupy greater prominence here in the future than it has done in the past, and towards a better knowledge of it the present volume must be regarded as a very important contribution."

British Medical Journal.- " The appearance of such a book is an important indication of the increasing space occupied by and the increasing importance, attached to considerations of hygiene in relation to disease. Its appearance is therefore welcome, and both the hygienist and the practitioner who study its contents will find much valuable practical information."

Scortish Medical Journal_- "Valuable as this work is the introduction will be read with the most unqualified appreciation by British readers. ... Taken as a whole, THE WORK IS ONE WHICH SHOUID BE CAREFULLY READ BY EVERY PERSON ON THE MEDICAL REGISTER." 


\title{
S U R G I A L A N A T O M Y
}

OF THE

\section{LYMPHATIC GLANDS}

BY

\author{
CECIL H. LEAF, F.R.C.S., etc.
}

\author{
WITH COLOURED DIAGRAMS
}

Price 10s. 6d. net.

\section{CONTENTS :}

Preface. Introduction. The Lymphatic Glands of the Head. The Lymphatic Glands of the Neck. The Cervical and Thoracic Lymphatic Glands. Thoracic Glands. Lymphatic Glands of the Axilla. Aortic and Sub-diaphragmatic Glands. Intestinal Glands. Pelvic Glands. Glands of the Groin. Popliteal Glands.

Edinburgh Medical Journal.- “ In the limited space of 72 pages, and with the aid of 18 admirable coloured plates, he has given in clear and concise language, all that it is important for us to know about the situation and relations of the various groups of glands. . . . We regard Mr. Leaf's small volume as an important contribution to anatomical and surgical literature."

Trmes. - " The Surgical Anatomy of the Lymphatic Glands by Mr. Cecil Leaf, M.B., F.R.C.S., is an illustrated description of the precise relation of the lymphatic glands of the human body to the neighbouring structures as these relations have been displayed by the employment of a new and improved method of preserving and preparing tissues in situ. The information conveyed will be helpful to surgeons in the performance of a large class of operations in which the entire removal of the glands in the vicinity of the diseased structures is a matter of vital importance."

Athenfum.- “Dr. Leaf's work will be serviceable to the surgeon because it shows him the exact position of the small lymphatic glands often hidden away in loose tissues near important vessels and nerves. . . . The illustrations are well drawn and well rendered."

Bristol Medico-Chirurgical Journal.- “ The object of the Author has been to indicate by a series of diagrams with descriptive text the position of the main groups of lymphatic glands in the human body, and to show the important bearing of this in surgical practice. . . The book well fulfils the aim of its Author, and we cordially recommend its study to those interested in this branch of anatomy." 

ir 
RETURN TO the circulation desk of any

University of California Library

or to the

NORTHERN REGIONAL LIBRARY FACILITY

Bldg. 400, Richmond Field Station

University of California

Richmond, CA 94804-4698

ALL BOOKS MAY BE RECALLED AFTER 7 DAYS

2-month loans may be renewed by calling

(510) 642-6753

1-year loans may be recharged by bringing books to NRLF

Renewals and recharges may be made 4 days prior to due date

DUE AS STAMPED BELOW

NOV 234992 
5 


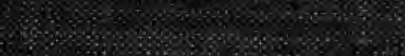

